238

ANL-7564

$12-12-69$
1126

ANL-7564

MASTER

Argonne Mational Laboratoru

FAST NEUTRONS INCIDENT ON VANADIUM

by

Alan B. Smith, James F. Whalen, and Kenii Takeuchi 


\section{DISCLAIMER}

This report was prepared as an account of work sponsored by an agency of the United States Government. Neither the United States Government nor any agency Thereof, nor any of their employees, makes any warranty, express or implied, or assumes any legal liability or responsibility for the accuracy, completeness, or usefulness of any information, apparatus, product, or process disclosed, or represents that its use would not infringe privately owned rights. Reference herein to any specific commercial product, process, or service by trade name, trademark, manufacturer, or otherwise does not necessarily constitute or imply its endorsement, recommendation, or favoring by the United States Government or any agency thereof. The views and opinions of authors expressed herein do not necessarily state or reflect those of the United States Government or any agency thereof. 


\section{DISCLAIMER}

Portions of this document may be illegible in electronic image products. Images are produced from the best available original document. 
The facilities of Argonne National Laboratory are owned by the United States Government. Under the terms of a contract (W-31-109-Eng-38) between the U. S. Atomic Energy Commission, Argonne Universities Association and The University of Chicago, the University employs the staff and operates the Laboratory in accordance with policies and programs formulated, approved and reviewed by the Association.

\section{MEMBERS OF ARGONNE UNIVERSITIES ASSOCIATION}

The University of Arizona Carnegie-Mellon University Casc Western Reserve University The University of Chicago University of Cincinnati Illinois Institute of Technology University of Illinois Indiana University Iowa Stato University The University of Iowa
Kansas State University The University of Kansas Loyola University Marquette University Michigan State University The University of Michigan University of Minnesota University of Missouri Nnrthwestern University University of Notre Dame
The Ohio State University Ohio University The Pennsylvania State University Purdue University Saint Louis University Southern Illinois University University of Texas Washington University Wayne State University The University of Wisconsin

\section{LEGAL NOTICE}

This report was prepared as an account of Government sponsored work. Noither the Uniter States, nor the Commission, nor any person acting on behalf of the Commission:

A. Makes any warranty or representation, expressed or implied, with respect to the accuracy, completeness, or usefulness of the information contained in this report, or that the use of any information, apparatus, method, or process disclosed in this report may not infringe privately owned rights; or

B. Assumes any liabilities with respect to the use of, or for damages resulting from the use of any information, apparatus, method, or process disclosed in this report.

As used in the above, "person acting on behalf of the Commission" includes any employee or contractor of the Commission, or employee of such contractor, to the extent that such employee or contractor of the Commission, or employee of such contractor prepares, disseminates, or provides access to, any information pursuant to his employment or contract with the Commission, or his employment with such contractor.

Printed in the United States of America

Available from

Clearinghouse for Federal Scientific and Technical Information National Bureau of Standards, U.S. Department of Commerce

Springfield, Virginia 22151

Price: Printed Copy $\$ 3.00$; Microfiche $\$ 0.65$ 
ARGONNE NATIONAL LABORATORY

9700 South Cass Avenue

Argonne, Illinois 60439

\section{FAST NEUTRONS INCIDENT ON VANADIUM}

by

Alan B. Smith, James F. Whalen, and Kenji Takeuchi

Reactor Physics Division

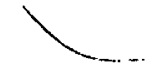

\section{LEGAL NOTICE}

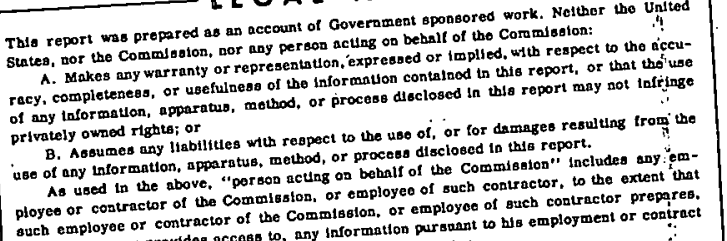

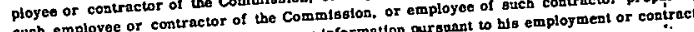

disseminates, or provides accoss wo, any iniormach contractor.

with the Comolission, or bis exploy

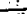

May 1969 


\section{THIS PAGE WAS INTENTIONALLY LEFT BLANK}


TABLE OF CONTENTS

$\underline{\text { Page }}$

i

ABSTRACT

I. INTRODUCTION.

II. EXPERIMENTAL METHOD

III. EXPERIMENTAL RESULTS.

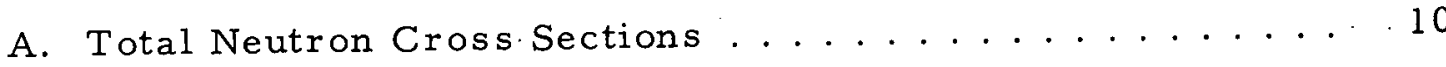

B. Elastic Cross Sections................... 11

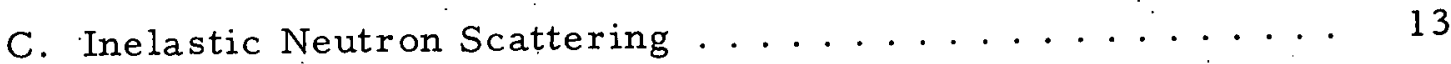

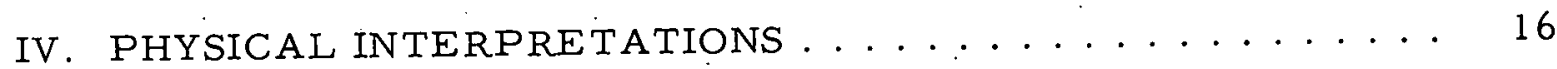

A. Optical Model and Statistical Calculations ......... 16

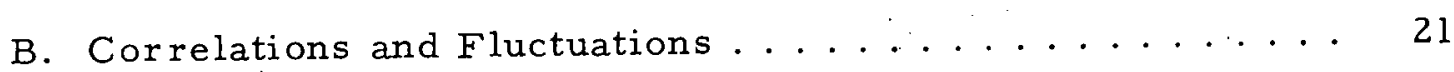

1. Existence of Intermediate Resonance Structure ..... 21

2. Limit of Strongly Overlapping Resonances, $\Gamma \gg>$ D. . . 22

3. Cross-section Fluctuations and Distributions of Widths

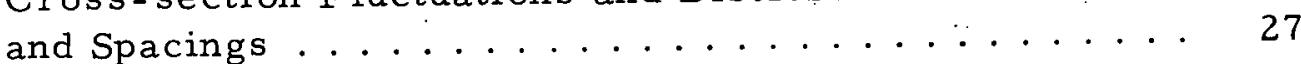

C. The Intermediate Optical Model............. 29

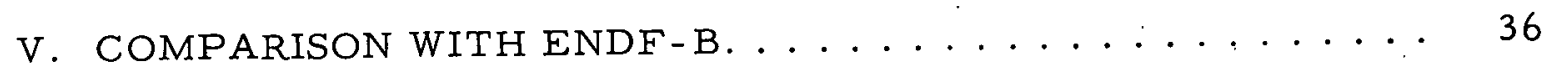

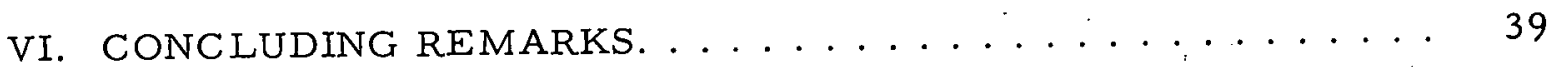

APPENDIX: Tabulations of Measured Cross Sections........ 41

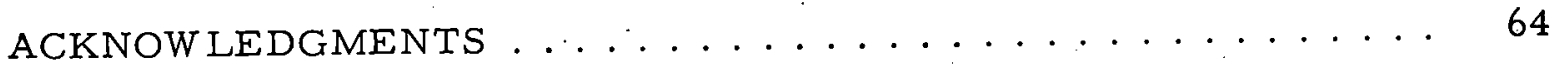

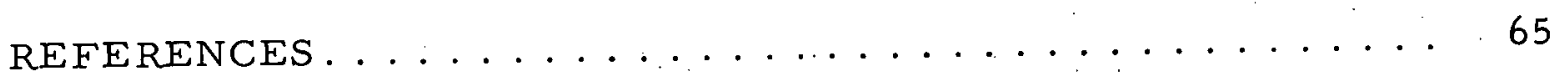




\section{LIST OF FIGURES}

No.

$\underline{\text { Title }}$

Page

1. Measured Total Neutron Cross Sections of Vanadium .......

2. Time-of-flight Spectra Obtained for Vanadium at a Laboratory Scattering Angle of $114^{\circ} \ldots \ldots \ldots \ldots \ldots$

3. Experimentally Observed Elastic and Inelastic Angular Distributions for Vanadium . . . . . . . . . . . . .

4. Differential Elastic Scattering Cross Sections of Vanadium Given in the Format of Eq. $1 \ldots \ldots \ldots \ldots$

5. Comparison of Experimental Total Neutron Cross Sections and Total Elastic Scattering Cross Sections of Vanadium ......

6. Measured Cross Sections for the Excitation of States in Vanadium at 0.32 and $0.93 \mathrm{MeV} \ldots \ldots \ldots \ldots$

7. Differential Cross Section for the Excitation of the $0.32-\mathrm{MeV}$ State in Vanadium, Expressed in the Form of Eq. 2 . . . . . .

8. Excited Structure of Vanadium . . . . . . . . . . . . 17

9. Comparison of Measured and Calculated Differential Elastic Distributions of Vanadium at Selected Incident Neutron Energies (keV).

10. Real and Imaginary Potential Values and Transmission Coefficients, $\mathrm{T}(\ell, \mathrm{j})$ Obtained by Fitting Eq. 3 to the Measured Elastic Scattering Cross Sections of Vanadium ...........

11. Measured and Calculated Elastic Distributions of Vanadium:. .

12. Calculated and Measured Cross Sections for the Excitation of the 0.32 - and $0.93-\mathrm{MeV}$ States in Vanadium. . . . . . . 18

13. Measured and Calculated Total Cross Sections of Vanadium...

14. Calculated Elastic and Inelastic Distributions of Vanadium and the Results Measured by Towle at $2.35 \mathrm{MeV}$. . . . . . . . . .

15. Elastic Angular Distribution of Vanadium Calculated Using the "Asymptotic Potential" Form of Eq. 3, Compared with the Measured Values of Becker at $3.0 \mathrm{MeV} \ldots \ldots . . . . .$.

16. Elastic Scattering from Vanadium at $7.05 \mathrm{MeV} \ldots \ldots 20$

17. Elastic Angular Distribution of Vanadium Measured at 14.7 MeV by Western et al., Compared with Results Calculated from Eq. $3 \ldots \ldots \ldots \ldots \ldots$ 
LIST OF FIGURES

No.

Title

Page

18. Function C(R), Eq. 5, Evaluated from the Measured Total

Neutron Cross Sections of Vanadium for $0<\mathrm{R}<480 \mathrm{keV}$. . .

19. $C(\delta)$ Evaluated from Total Neutron Cross Sections of

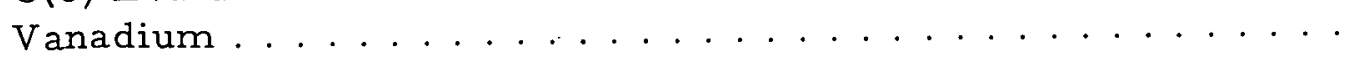

20. Measured Total Neutron Cross Sections of Vanadium ......

21. Differential Elastic Neutron Scattering Cross Sections of

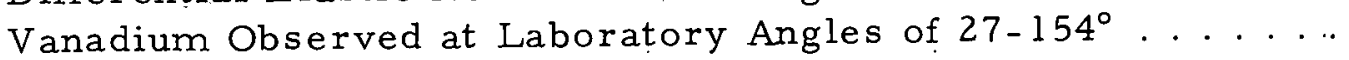

22. Real and Imaginary Portions of the Intermediate Optical Potential; Compared with the Phenomenological Optical Potential Derived from a Fit to the Measured Elastic Scattering Distributions . . . . . . . . . . . . . . . . . .

23. Comparison of Experimental Elastic Scattering Angular Distributions and Inelastic Cross.Sections of Vanadium with Those Calculated from the Intermediate Optical Potential and

Statistical Theory. . . . . . . . . . . . . .

24. Total Cross Sections of Vanadium Derived from Present Work and ENDF-B Data. . . . . . . . . . . . . . . .

25. Total Cross Sections, Calculated as Described in Section IV.A, Compared with ENDF-B Values . . . . . . . . . . .

26. Elastic Scattering Cross Sections of Vanadium Determined in the Present Work and the Values Given by ENDF-B.......

27. Measured and ENDF-B Inelastic Excitation Cross Sections of

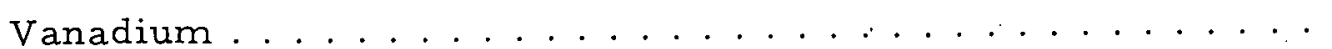


LIST OF TABLES

No.

$\underline{\text { Title }}$

$\underline{\text { Page }}$

I. Results of Correlation Analysis of Measured Total Cross

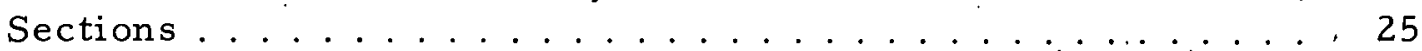

II. Cross-correlation Values ................ 26

III. Comparison of Experimentally and Theoretically Derived Level Densities .................... . . 28

IV. Doorway Potential Parameters Used with Eq. $20 \ldots \ldots . \ldots$

V. Observed Total Neutron Cross Sections of Vanadium ......4 41

VI. Differential Elastic Scattering Cross Sections of Vanadium Expressed in Format of Eq. 1. . . . . . . . . . . . . 55

VII. Cross Sections for the Inelastic Excitations of the $320-\mathrm{keV}$ State in Vanadium by Neutron Scattering. ...........66 61

VIII. Cross Sections for the Inelastic Neutron Excitations of the $930-\mathrm{keV}$ State in Vanadium. . . . . . . . . . . . . . . . 


\title{
FAST NEUTRONS INCIDENT ON VANADIUM
}

\author{
by
}

Alan B. Smith, James F. Whalen, and Kenji Takeuchi

\begin{abstract}
Total neutron cross sections and elastic and inelastic neutron scattering cross sections are experimentally studied. Total neutron cross sections are determined with good resolution ( $\geqq 1 \mathrm{ke} \cdot \mathrm{V}$ ) from 0.1 to $1.45 \mathrm{MeV}$. Differential elastic and inelastic scattering angular distributions are measured at incident neutron energy intervals of $\lesssim 10 \mathrm{keV}$ from 0.3 to $1.5 \mathrm{MeV}$ with incident neutron resolutions of $\sim 20 \mathrm{keV}$. The inelastic excitation of states at $33.0 \pm 10 \mathrm{keV}$ and $926 \pm 10 \mathrm{keV}$ is observed. The experimental results are interpreted in terms of the optical model and statistical concepts, inclusive of resonance width fluctuations and correlations. The observed intermediate structure is discussed in the context of strongly overlapping resonances and distributions in resonance widths and spacings and in terms of an intermediate optical model. Comparison is made with previously reported experimental values and with the pertinent contents of the evaluated data file, ENDF-B. Numerical tabulations of the experimental results are provided.
\end{abstract}

\section{INTRODUCTION}

The objective of this study was to improve the understanding of total neutron cross sections and of the elastic and inelastic scattering cross sections of vanadium at incident energies up to $1.5 \mathrm{MeV}$. Natural vanadium is monoisotopic, lies near the peak of the s-wave strength function, is magic in neutron number, and has relatively low-lying states, which are appreciably excited by inelastic neutron scattering processes. These collective properties are unusual, and many of them have been associated with the prevalence of intermediate structure in neutron processes. ${ }^{1}$ The relatively large inelastic excitations make possible the comparison of such intermediate structure between several reaction channels. The present work gave considerable attention to the experimental accuracies and energy resolutions in an effort to well define the energy-dependent structure. 
In the discussion, Section II outlines the techniques used in the experimental portions of the study. The experimental results are presented in Section III and comparisons made with previously reported values. Section IV is devoted to the physical interpretation of the measured results. A broad energy average of the measured values is described in terms of a conventional optical potential ${ }^{2}$ and statistical concepts ${ }^{3}$ inclusive of resonance width fluctuations. ${ }^{4}$ The observed intermediate structure is discussed in the context of (a) strongly overlapping compound nucleus resonances, ${ }^{5}$ (b) fluctuations in widths and spacings of isolated or partially overlapping resonances, ${ }^{6,7}$ and (c) an intermediate optical potential assuming reaction processes proceeding through "doorway" states. ${ }^{8,9}$ Section V compares the results of the-present study with the comparable physical quantities in the evaluated data file ENDF-B. ${ }^{10}$ This comparis on may prove of interest to the reactor physicist. Section VI consists of some brief summary remarks. The appendix presents experimental results in detailed tabular form. 


\section{EXPERIMENTAL METHOD}

Fast-neutron time- of-flight techniques were used in all the scattering measurements and a portion of the total cross-section determinations. The experimental neutron-scattering apparatus, inclusive of a pulsed and bunched Van de Graaff accelerator, a multiangle detection system, on-line computer usage, and associated data-processing procedures, has been described in detail and will not be further defined here. ${ }^{11,12}$ All measurements used the reaction ${ }^{7} \mathrm{Li}(\mathrm{p}, \mathrm{n})^{7} \mathrm{Be}$ as a neutron source, the intensity of which was monitored using "long counters" and proton-recoil scintillators. ${ }^{13}$ The scattering samples were right cylinders $2.0 \mathrm{~cm}$ in diameter and $2.0 \mathrm{~cm}$ high) fabricated of natural vanadium metal. The chemical purity of the metal was $\gtrsim 98 \%$. Neutrons were incident upon the lateral surfaces of the scattering samples. All scattering measurements were made relative to the known differential elastic scattering cross sections of carbon and corrected for multiple scattering, incident-beam attenuation, and angular-resolution effects. ${ }^{11,14}$

Total neutron cross sections were determined from neutron transmissions measured using a pseudowhite pulsed-source technique or the monoenergetic source method. ${ }^{12,15}$ The latter method was used exclusively below incident neutron energies of $\sim 500 \mathrm{keV}$. All neutron transmission measurements and reduction to total cross sections were carried out in an automated manner as described in Ref. 12. Transmission samples of natural vanadium varied in geometry, but generally were such as to provide transmissions of greater than $50 \%$. 


\section{EXPERIMENTAL RESULTS}

\section{A. Total Neutron Cross Sections}

The experimental total cross-section results in the measured interval 0.1-1.45 MeV are shown in the upper portion of Fig. 1. Numerical values are given in Table $\mathrm{V}$ (in the appendix). At incident neutron energies of $<0.5 \mathrm{MeV}$, the experimental energy resolution was $1.5-2.5 \mathrm{keV}$. At higher energies, the velocity resolution was estimated to be $0.1-0.12 \mathrm{nsec} / \mathrm{m}$, from direct observation of the prompt gamma-ray burst and the width of narrow resonance peaks. The energy scale of the monoenergetic measurements was calibrated against the ${ }^{7} \mathrm{Li}(\mathrm{p}, \mathrm{n})^{7} \mathrm{Be}$ reaction threshold of $1.882 \mathrm{MeV} \cdot{ }^{16}$ Energy calibration errors in this method should have been less than $3 \mathrm{keV}$. The time-of-flight measurements were calibrated against the velocity of light and spot-checked using the above monoenergetic method. The error in the time-of-flight energy scale was judged appreciably less than $10 \mathrm{keV}$, with the larger uncertainties at the higher energies. The stated crosssection errors were derived from counting statistics. "In scattering" corrections were estimated, found small, and neglected.

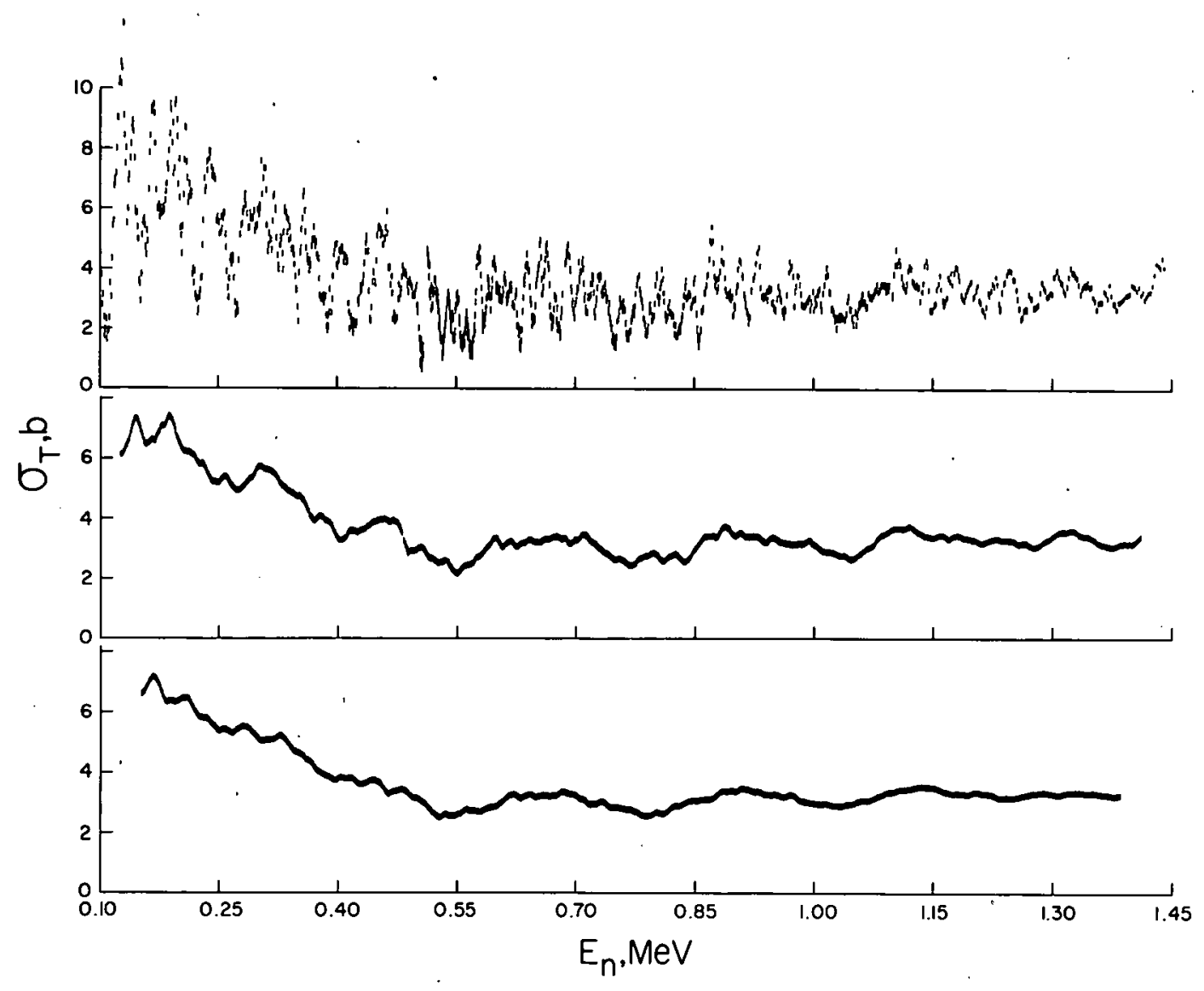

112-9505 Rev. 1

Fig. 1. Measured Total Neutron Cross Sections of Vanadium. Top, basic measured data; center, data averaged over a $50-k e v$ interval; bottom, data averaged over a $100-\mathrm{keV}$ interval. 
Generally, the present results compared favorably with those obtained by other workers. ${ }^{17}$. Values reported by Cabe et al., in the interval 0.4-1.2 MeV follow the energy average of the present higher-resolution values. ${ }^{18}$ The present work is consistent with the detailed results of Rohr and Friedland and of Firk et al., reported for incident neutron energies of $\lesssim 200 \mathrm{keV} .^{19,20}$

\section{B. Elastic Cross Sections}

The differential elastic neutron-scattering cross sections were determined at incident neutron-energy intervals of $\lesssim 10 \mathrm{keV}$ with an incident neutron-energy resolution of $20 \pm 5 \mathrm{keV}$. The angular range of the measurements at each incident energy was from $\sim 25$ to $\sim 155^{\circ}$, usually covered in eight angular intervals. Typical values of the laboratory scattering angles were $27,38,53,69,84,114,129$, and $154^{\circ}$. The scattering angles were not always identical, but were carefully determined at each measurement. Angular resolutions varied, but were of the order of a few degrees. As indicated by the illustrative time spectra of Fig. 2, the scattered neutron velocity resolution of $1.5-2.0 \mathrm{nsec} / \mathrm{m}$ was sufficient to resolve the elastic scattered neutrons from inelastic scattered components.

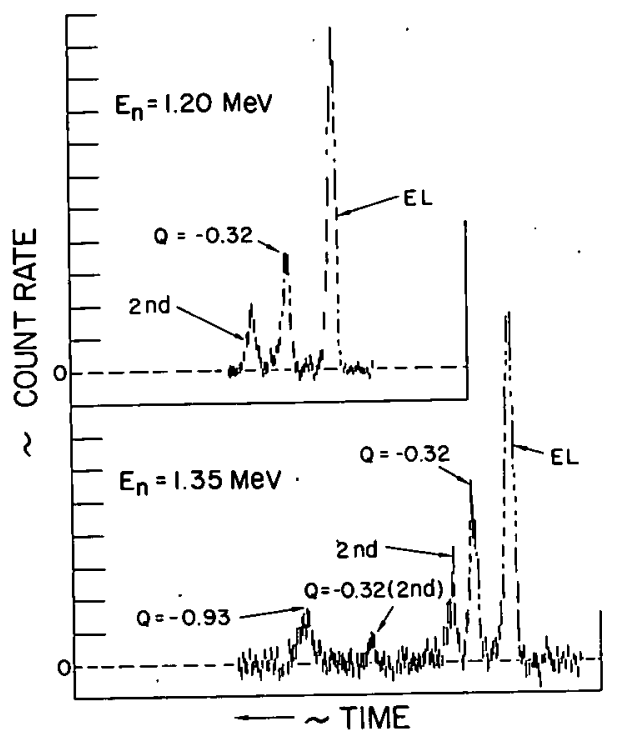

113-494 Rev. 1

Fig. 2

Time-of-flight Spectra Obtained for Vanadium at a Laboratory Scattering Angle of $114^{\circ}$. Flight path was $\sim 203 \mathrm{~cm}$, and time per channel $\sim 1.05 \mathrm{nsec}$. Elastic and inelastic scattered neutron groups are evident. Backgrounds have been subtracted, and vertical bars indicate standard deviation of respective points. No detector-sensitivity correction has been made. Reaction $Q$ values associated with the various neutron groups are indicated in $\mathrm{MeV}$.

The experimental results were expressed in the following alternate forms:

$$
\frac{\mathrm{d} \sigma}{\mathrm{d} \Omega}=\frac{\sigma}{4 \pi}\left[1+\sum_{\mathrm{i}=1}^{4} \omega_{\mathrm{i}} \mathrm{P}_{\mathrm{i}}\right] \text {, }
$$

and

$$
\}
$$

$$
\frac{\mathrm{d} \sigma}{\mathrm{d} \Omega}=\sum_{i=0}^{4} \mathrm{~B}_{\mathrm{i}} \mathrm{P}_{\mathrm{i}},
$$


where $P_{i}$ are Legendre polynomials expressed in the laboratory system and $\sigma, \omega$, and $B$ are parameters derived by least-squares fitting Eq. 1 to experimentally measured differential distributions. The expansions of Eq. 1 were terminated at $\mathrm{i}=4$, as the magnitudes of higher-order terms did not generally exceed their corresponding uncertainties. Expressed in either of the above forms, the results were descriptive of the measured data points (see Fig. 3), and parameter values obtained from measurements at slightly different incident energies or scattering angles were easily comparable. However, extrapolation of the results, expressed in the forms of Eq. 1, beyond the measured angular interval of $\sim 25$ to $\sim 155^{\circ}$, is not necessarily valid.

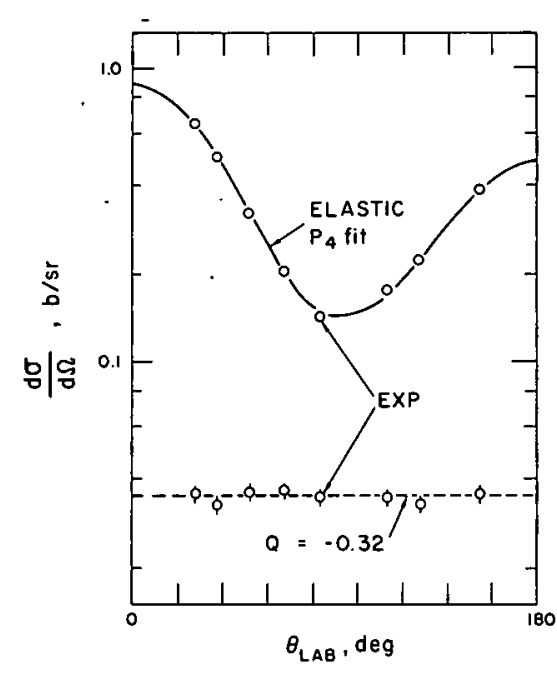

Fig. 3

Experimentally Observed Elastic and Inelastic Angular Distributions for Vanadium (data points). Solid curve is obtained from the least-squares fit of Eq. 1 to the experimental elastic scattering measurements. The dotted curve is an eye guide to the inelastic scattering angular distribution.

As expected from the multiresonance nature of the total neutron cross section (see Fig. 1), the elastic scattering cross sections varied rapidly with incident energy. Small experimental variations in incident neutron energy and/or energy spread could, and did, result in quite different measured cross-section values. To provide consistent experimental results, the measured differential elastic cross sections were progressively averaged over a square energy increment with a width of several experimental resolutions. Figure 4 shows results obtained with a $50-\mathrm{keV}$ averaging increment. Table VI (in the appendix) lists the numerical parameter values derived from the differential measurements. Assay of the experimental errors was complex and subjective. Generally, it was estimated that the elastic scattering cross sections were determined to an accuracy of $\sim 8 \%$, inclusive of uncertainties in the cross section of the carbon standard. The stated uncertainties in the angular-distribution coefficients represent standard deviations derived from the least-squares fitting procedures.

The elastic scattering results were generally consistent with the total cross section (see Section A above) as indicated in Fig. 5 and with the reported total scattering results of Ref. 14. The agreement between the present results and those obtained using a soft fission spectrum ${ }^{21}$ was less satisfactory. 


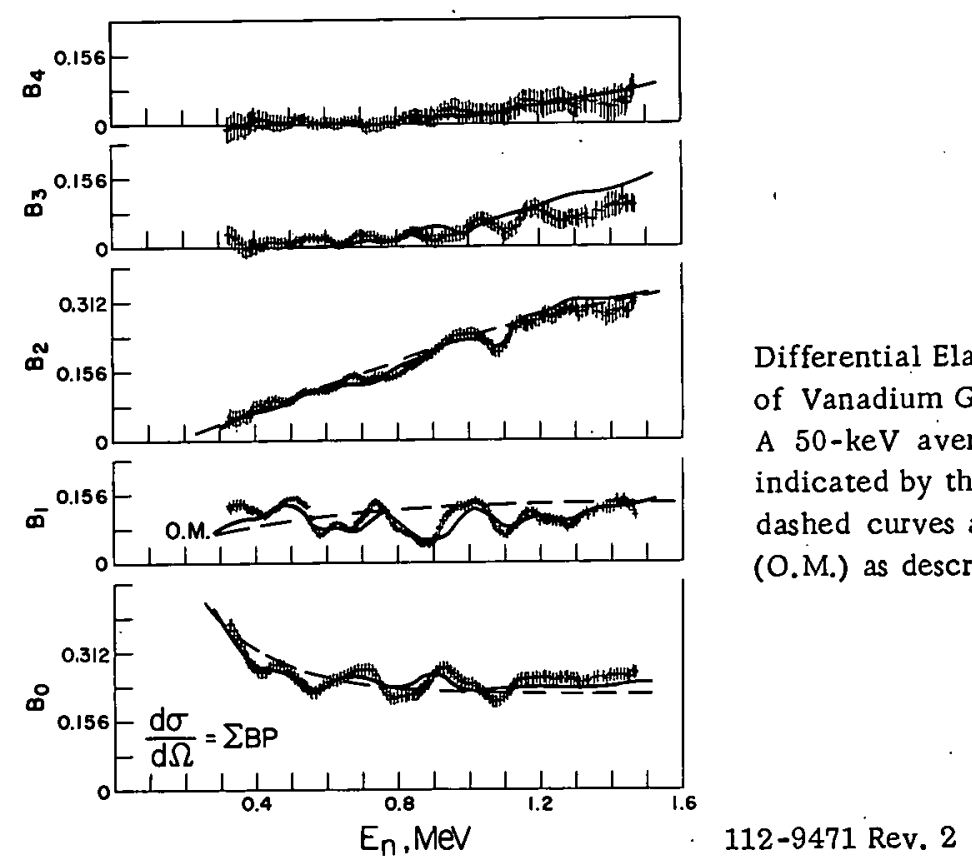

Fig. 4

Differential Elastic Scattering Cross Sections of Vanadium Given in the Format of Eq. 1 . A $50-\mathrm{keV}$ average of the measurements is indicated by the data points. The solid and dashed curves are the result of calculation (O.M.) as described in Section IV.A.

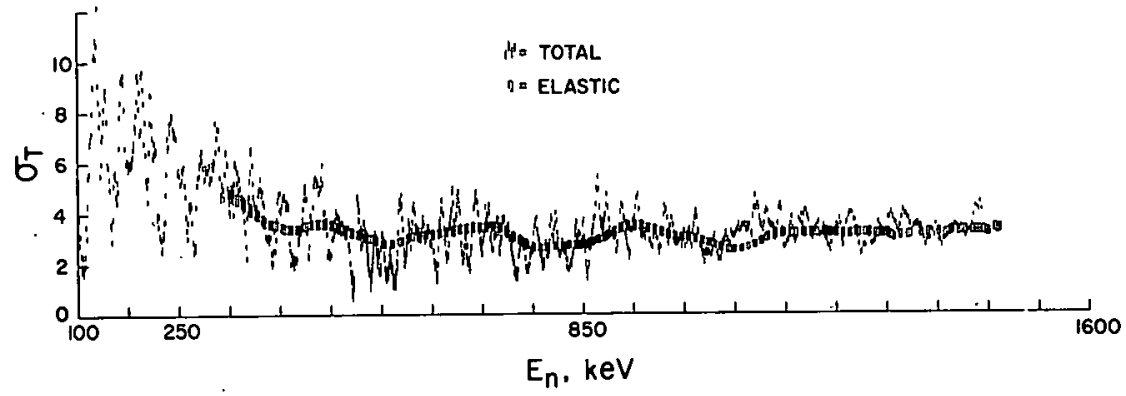

112-9503 Rev. 1

Fig. 5. Comparison of Experimental Total Neutron Cross Sections (vertical bars) and Total Elastic Scattering Cross Sections (boxes) of Vanadium

C. Inelastic Neutron Scattering

The inelastic excitation of states in vanadium at $330 \pm 10$ and $926 \pm 10 \mathrm{keV}$ was observed. The energy values were derived from the calibrated time scale of the velocity spectrometer and verified by observation of the excitation of the well-known $845-\mathrm{keV}$ state in iron, ${ }^{22}$ These levels were attributed to reported states at 320 and $930 \mathrm{keV}$, respectively. ${ }^{22}$ The latter published values were accepted as the more accurate. Additional. states in vanadium have been reported at 480,645 , and $1160 \mathrm{keV} .{ }^{22-24}$ In the se experiments, any state at $\sim 480 \mathrm{keV}$ would have been partly obscured by the presence of elastically scattered neutrons from the second neutron group of the ${ }^{7} \mathrm{Li}(\mathrm{p}, \mathrm{n})^{7} \mathrm{Be}$ source reaction. ${ }^{16}$ However, it was felt that a cross section for the excitation of a $480-\mathrm{keV}$ state of $\gtrsim 10.0 \mathrm{mb} / \mathrm{sr}$ would have resulted in an observable inelastically scattered neutron group. None was evident. Inelastic scattered neutrons $(Q=-0.32 \mathrm{MeV})$ resulting from the second source group were clearly seen (see Fig. 2). A nearby neutron 
group at a somewhat higher scattered neutron energy due to the excitation of a $645-\mathrm{keV}$ state would have been observed if the respective cross section was $\gtrsim 2 \mathrm{mb} / \mathrm{sr}$. No group corresponding to such a magnitude was observed. The experimental results indicated an upper limit of $\sim 4 \mathrm{mb} / \mathrm{sr}$ for the excitation of any state in the vicinity of $1.16 \mathrm{MeV}$. Within this limitation, there was no evidence for such a level. Thus there was no positive evidence in the present experiments for states in vanadium at approximately 480,645 , and/or $1160 \mathrm{keV}$. If present, such states probably have spins and parities requiring $l \geq 2$ incident neutron momentum for appreciable excitation. This conclusion is consistent with the results of recent inelastic neutron-scattering measurements at higher incident energies ${ }^{25}$ and with the results of recent shell-model calculations. ${ }^{26}$

The differential cross sections for the excitation of the 320 - and $930-\mathrm{keV}$ states were measured from several hundred $\mathrm{keV}$ of threshold to a maximum incident energy of $1.5 \mathrm{MeV}$. Generally the measurements were made at the same scattering angles as employed in the elastic scattering studies. Many of the measured inelastic angular distributions were essentially isotropic. (See Fig. 3, for example.) Thus the angle-integrated inelastic-excitation cross sections were reasonably obtained by averaging the differential measurements and multiplying by $4 \pi$. Even for the more anisotropic distributions observed, this approximate procedure was judged acceptable since it led to overestimates of the excitation cross sections by $\lesssim 5 \%$. The resulting angle-integrated excitation cross sections are shown in Fig. 6 and listed in Tables VII and VIII (in the appendix). As was true for the elastic cross sections, the results varied with energy because of the partially resolved resonance structure and experimental uncertainties in the precise incident energy and incident-energy spread. The uncertainties associated with the individual cross-section measurements are complex composites of statistical and instrumental effects and were subjectively estimated. Those indicated in Fig. 6 and Tables VII and VIII are best estimates of the standard deviation inclusive of uncertainties in the reference standard cross section of carbon.

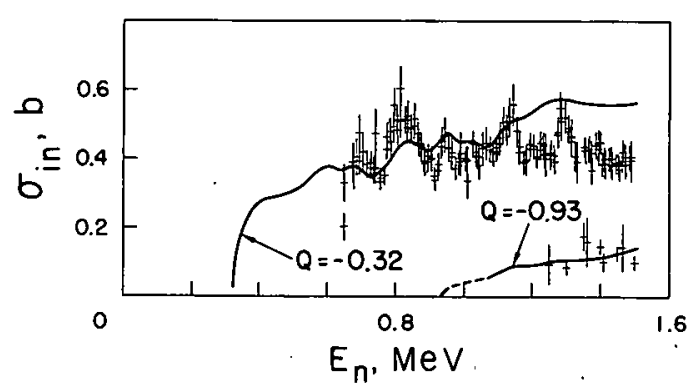

Fig. 6

Measured Cross Sections for the Excitation of States in Vanadium at 0.32 and $0.93 \mathrm{MeV}_{3}$ (crosses). The solid curve was obtained by calculation as described in Section IV.A. Estimated experimental uncertainties are indicated by vertical bars of the data crosses. 113-501 Rev. 1

The measured differential cross sections for the excitation of the $320-\mathrm{keV}$ state were carefully assayed and the "better" angular distributions fitted, by the method of least squares, with the expression

$$
\frac{\mathrm{d} \sigma}{\mathrm{d} \omega}=\mathrm{B}_{0}+\mathrm{B}_{1} \mathrm{P}_{1}+\mathrm{B}_{2} \mathrm{P}_{2}
$$


The resulting $B_{i}$ parameters were found to be very energy-dependent and were smoothed by averaging over a $20 \mathrm{keV}$ incident energy increment.

Figure 7 shows the resulting average
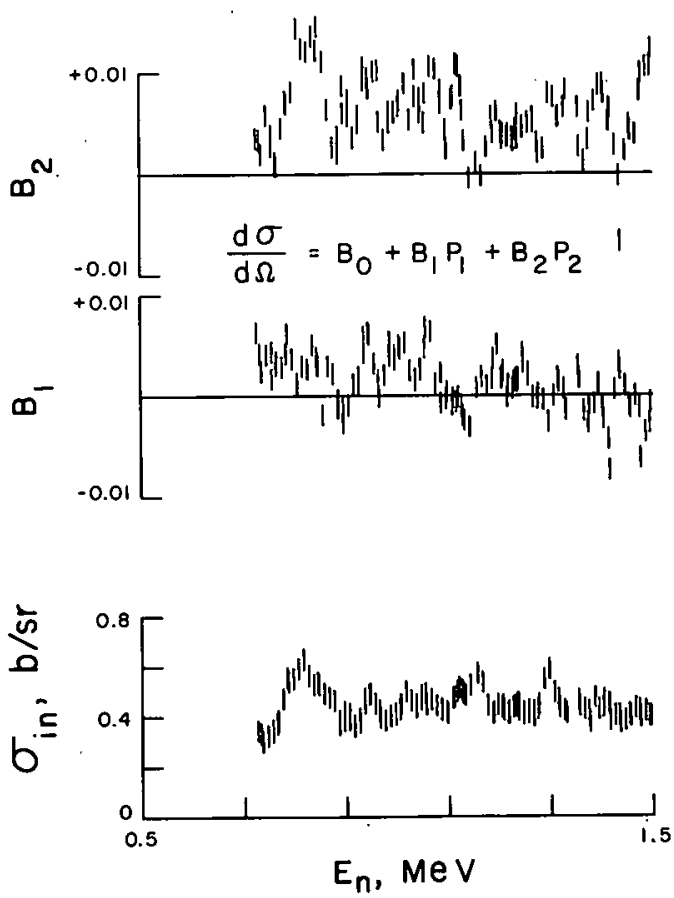

113-523 Rev. 1

Fig. 7

Differential Cross Section for the Excitation of the $0.32-\mathrm{MeV}$ State in Vanadium, Expressed in the Form of Eq. 2. Lowest plot: integrated cross section. Upper two plots: $B_{1}$ and $B_{2}$ coefficients of a Legendre expansion of the differential distributions. Data points (bars) do not indicate experimental uncertainties. energy dependence of these parameters. Though the parameters still vary appreciably, it is evident that (a) the cross section $\left(\sigma=4 \pi B_{0}\right)$ has the same magnitude and energy dependence as that shown in Fig. 6, and (b) $B_{1}$ tends to be less than $B_{2}$, both on the average and in magnitude of the fluctuations. Further, there appears to be a qualitative correlation between fluctuations in $B_{2}$ and $B_{0}$. Instrumental effects may have contributed to the structure and anisotropy. Such perturbations were most likely at forward angles; thus most affect the $B_{1}$ coefficient. Such a premise is not strongly supported by the results of Fig. 7. Coincidence may have led to measurements locally correlated with compound-nucleus resonancè structure, resulting in systematic perturbation of measured magnitudes or angular distributions. The abundance of data and the random mode of its procurement over a several-year period made this hypothesis unlikely.

The present results can be compared with the few previously reported values. Towle reports cross sections for the excitation of the 320 - and $930-\mathrm{keV}$ states at incident energies of $\sim 1.5 \mathrm{MeV}$ similar to the results of the present work. ${ }^{25}$ Results of $\left(n: n^{\prime} \gamma\right)$ measurements by Mather et al., at $0.8,1.0$, and $1.5 \mathrm{MeV}$ are consistent with the values obtained in the present work when cognizance is taken of the anisotropy of the emitted quanta and the effects of branching ratios. ${ }^{27}$ Barrows reports ( $n^{\prime} n^{\prime} \gamma$ ) results at incident energies of $1.83 \mathrm{MeV}$ and above. ${ }^{28}$ When extrapolated downward to the $1.5-\mathrm{MeV}$ maximum energy of the present work, Barrows' results are slightly $(\sim 20 \%)$ higher than those reported here. This may in part be due to the use of the rapidly varying $F e\left(n ; n^{\prime} \gamma\right)(Q=-0.85 \mathrm{MeV})$ cross section as a reference standard in Barrows' work. 


\section{PHYSICAL INTERPRETATIONS}

A. Optical Model and Statistical Calculations

The optical model and statistical methods were used in the phenomenological interpretation and extrapolation of the experimental results. ${ }^{2,3,30,31}$ The potential employed was based upon the surface absorption model of Moldauer. ${ }^{32}$ The latter has been extended by Engelbrecht and Fiedeldey to an equivalent nonlocal potential of the for $\mathrm{m}^{29}$

$$
\left.V_{c}(r)=-(V+i U) f_{1}(r)-i f_{2}(r) W+V_{s o}\left(\frac{\hbar}{\mu_{\pi} c}\right)^{2} \underline{\sigma} \cdot \underline{\ell} \frac{1}{r} \frac{d f_{1}}{d r},\right]
$$

where

$$
\begin{aligned}
f_{1}(r) & =\left[1+\exp \left(\frac{r-R_{1}}{a_{1}}\right)\right]^{-1}, \\
f_{2}(r) & =\exp \left[-\left(\frac{r-R_{2}}{a_{2}}\right)^{2}\right], \\
R_{1} & =r_{1}+r_{0} A^{1 / 3}, \quad R_{2}=R_{1}+r_{2}, \\
r_{0} & =1.16 f, \quad r_{1}=0.6 f, \quad r_{2}=0.5 f, \\
a_{1} & =0.62 f, \quad a_{2}=0.5 f, \\
V^{*} & =V_{0}-0.25 E, \\
W^{*} & =W_{0}-0.2 \mathrm{E}, \\
V_{s_{0}} & =7.0,
\end{aligned}
$$

and

$$
U^{*}=0.125 E-0.0004 E^{2} \text {. }
$$

If we let $\mathrm{E} \rightarrow 0$ and $\mathrm{V} \rightarrow 0$ and set $\mathrm{V}_{0}=46$ and $\mathrm{W}_{0}=14 \mathrm{MeV}$, Eq. 3 becomes the potential of Moldauer. This potential and the Hauser-Feshbach formal- ism give a good description of the low-energy observed elastic scattering (dashed curves of Fig. 4) and lead to calculated $l=0$ strength functions similar to those experimentally observed. ${ }^{34}$

A detailed description of the observed structure in the elastic scattering was sought by varying $\mathrm{V}_{0}$ and $\mathrm{W}_{0}(\mathrm{U}=0$, and other parameters maintained constant) to obtain the best fit to the individual observed elastic scattering angular distributions. ${ }^{33}$ The calculated results included compound

\footnotetext{
* All energies in $\mathrm{MeV}$.
} 


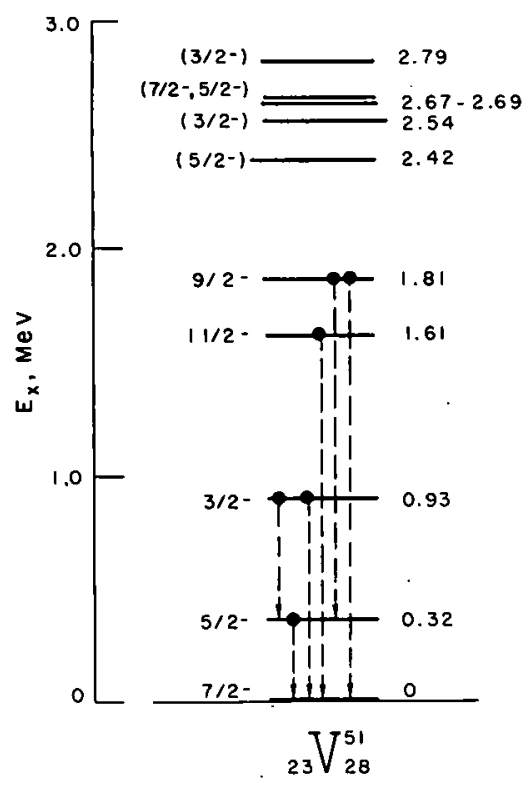

$112-9572$

Fig. 8. Excited Structure of Vanadium 22 elastic scattering derived with the Hauser $-F$ eshbach formula and the reported excited structure of vanadium $^{22}$ (see Fig. 8). The angular distributions calculated using this fitting procedure were descriptive of measurement (Fig. 9) and followed the observed intermediate structure of the elastic scattering (see curves of Fig. 4). The $V_{0}$ and $W_{0}$ and associated transmission coefficients obtained from the fitting reflected the intermediate structure, fluctuating with energy as shown in Fig. 10.

The meaning of the above fluctuating parameters was not above question. However, above energies of about $1.1 \mathrm{MeV}, \mathrm{V}_{0}$ and $\mathrm{W}_{0}$ obtained from the fitting procedure approached asymptotic values of 43 and $8 \mathrm{MeV}$, respectively. Elastic scattering cross sections calculated from these "asymptotic parameters" were in reasonable agreement with experiment above $\sim 0.8 \mathrm{MeV}$ (see solid curves of Fig. 11), but the agreement degenerated at lower energies, and the calculated $l=0$ strength function was about half that reported from microscopic measurement.

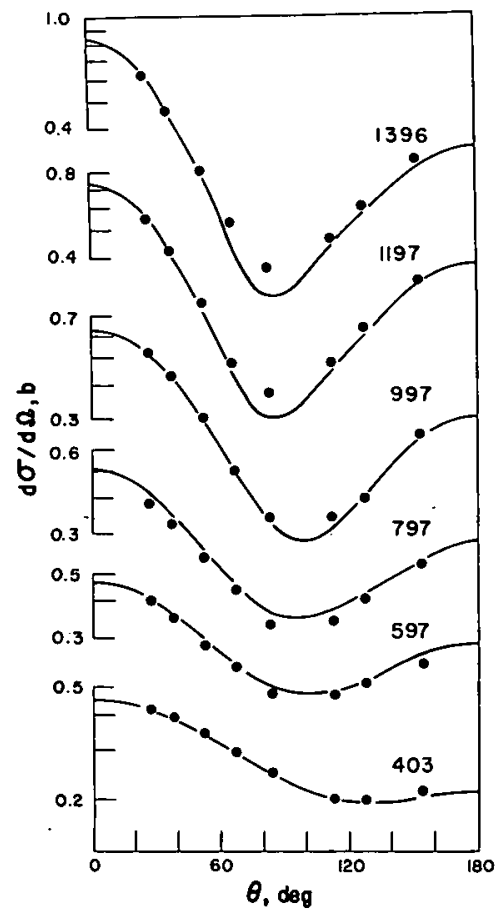

$112-9574$ Rev. 1

Fig. 9. Comparison of Measured (points) and Calculated (lines) Differential Elastic Distributions of Vanadium at Selected Incident Neutron Energies (keV)

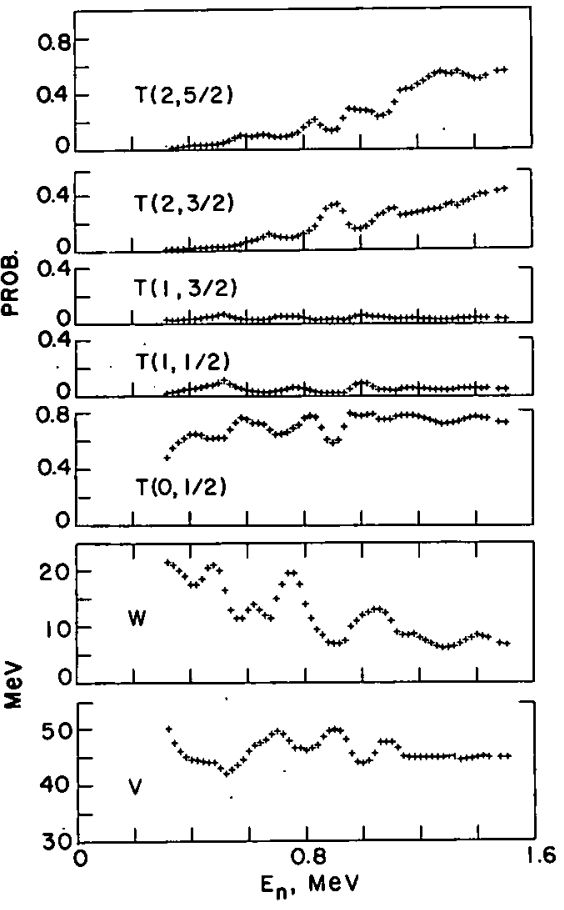

112-9506 Rev. 1

Fig. 10. Real (V) and Imaginary (W) Potential Values and Transmission Coefficients, $T(\ell, j)$ Obtained by Fitting Eq. 3 to the Measured Elastic Scattering Cross Sections of Vanadium. See Section IV.A for discussion. 

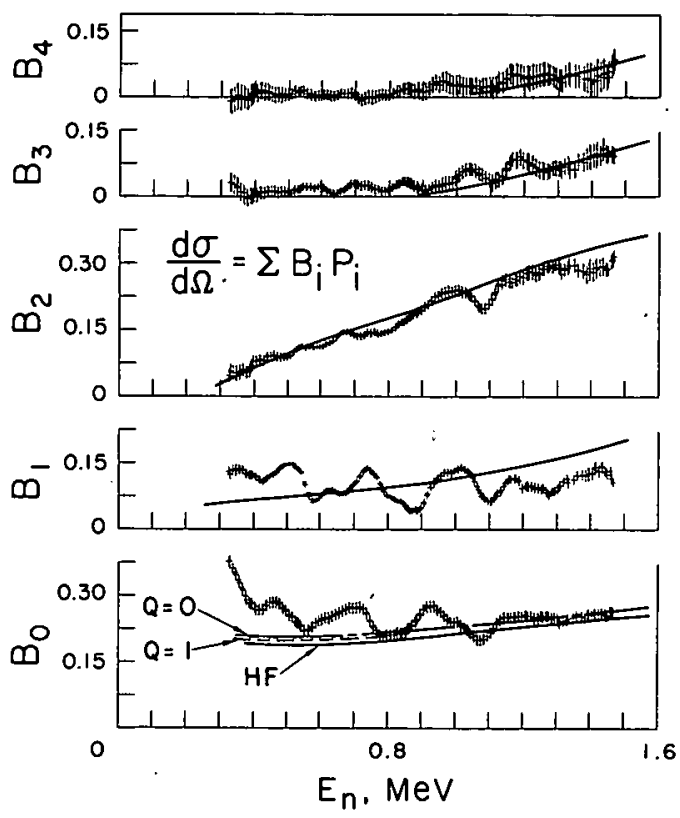

$1.13-498$

Fig. 11. Measured (crosses) and Calculated (curves) Elastic Distributions of Vanadium. The "asymptotic potential" form of Eq. 3 was used. The HauserFeshbach formalism was employed to estimate compound elastic contribu tions. Curves denoted as $Q=0$ and $\mathrm{Q}=1$ were obtained by applying correction factors as described in Section IV.A. All calculated results lead to essentially identical $B_{1} \rightarrow B_{4}$ coefficients.
The fluctuating potential parameters obtained from the above fitting procedures and the Hauser-Feshbach formula were used to calculate the inelastic-excitation cross sections; the results are shown in Fig. 6. The calculated values qualitatively display structure similar to that experimentally observed, though the calculation was based upon the potential obtained entirely from the fit to the elastic data. Inelastic-excitation cross sections calculated from the "asymptotic potential" $\left(V_{0}=43, W_{0}=8\right)$ were in fair agreement with experiment at energies above $\sim 1.0 \mathrm{MeV}$ (solid curves of Fig. 12), but were less descriptive at lower energies. The low-energy discrepancies tended to be independent of potential and were evident in other reported calculations employing different potentials. ${ }^{25,68}$ Results of calculations made with changes of one unit of spin for both the 320 - and the 930-keV states compared poorly with the experimental results. This, together with the previously reported evidence, ${ }^{25}$ made it difficult to accept spin-and-parity assignments differing from those shown in Fig. 8.

Compound nucleus resonance widths fluctuate in a statistically describable manner, and transmission coefficients are no longer described by $\mathrm{T}=2 \pi\langle\Gamma\rangle \mathrm{D}^{-1}$ where $\mathrm{T} \rightarrow 1$. The effects of such resonancewidth fluctuation and interference have been extensively discussed by Moldauer. ${ }^{4,33,35}$ He derived a "corrected" transmission coefficient, $\theta_{\alpha}$, of the form

$\theta_{\alpha}=2 Q_{\alpha}^{-1}\left[1-\left(1-Q_{\alpha} T_{\alpha}\right)^{1 / 2}\right]$.

For well-isolated resonances $\Gamma<<$ $\mathrm{D}, \mathrm{T}$ is small and $\theta \rightarrow \mathrm{T}$. Using the "asymptotic potential" and the correction factors of Eq. 4, we calculated both elastic and inelastic cross sections for the limiting

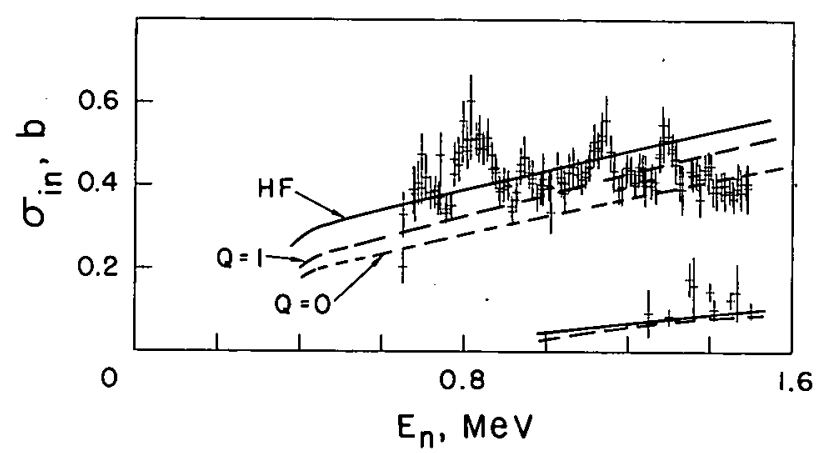

$113-500$

Fig. 12. Calculated (curves) and Measured (crosses) Cross Sections for the Excitation of the 0.32 - and $0.93-\mathrm{MeV}$ States in Vanadium. The interpretation of the calculated curves is discussed in Section IV.A. 
cases $Q=1$ (isolated resonances) and $Q=0$ (strongly overlapping resonances). The results are shown in Figs. 11 and 12. Results obtained with intermediate choices of the $Q$ parameter lay between the limits set by the above extreme cases. The correction factors and choice of the $Q$ parameter did not lead to calculated elastic scattering differing appreciably from that obtained with the Hauser-Feshbach formalism above. The effect of the correction factors on the calculated inelastic cross sections was appreciable (see Fig. 12), the corrected values lying 20-30\% below the Hauser-Feshbach results and being in poorer agreement with experiment. These qualitative effects of the correction factors were not appreciably dependent upon the choice of the optical potential. Generally, comparison with present experiments gave little guidance as to desirability or magnitude of correction terms, though comparisons by other workers at higher energies indicated the desirability of the fluctuation correction. ${ }^{25}$

It is interesting to examine the applicability of the "asymptotic potential" over a wide energy range, since the parameters of Eq. 3 are energy-dependent, and the volume absorption becomes significant at higher energies. The calculated total neutron cross section compares well with experiment to $10.0 \mathrm{MeV}$ (present work and Ref. 36), as illustrated in Fig. 13. The calculated elastic angular distributions are in agreement with experimental results reported at incident energies of $2.35,3.0$, and $7.05 \mathrm{MeV}$, as shown in Figs. 14,15 , and 16 , respectively. ${ }^{25,37,38}$ At $14.7 \mathrm{MeV}$, the agreement with experiment ${ }^{39}$ is less satisfactory (see Fig. 17), although. it is not clear whether the discrepancies are due to deficiencies in the calculation or experiment or both. Inelastic scattering calculated with the "asymptotic potential" and the Hauser-Feshbach formalism was in relatively good agreement with values measured ${ }^{25}$ at $2.35 \mathrm{MeV}$ (see Fig. 14). Generally, the form of Eq. 3 with the "asymptotic potential" parameters appears widely applicable to the calculation of total, elastic, and inelastic cross sections of vanadium at energies above about $0.8 \mathrm{MeV}$. The model is less successful at lower energies and certainly is not unique.

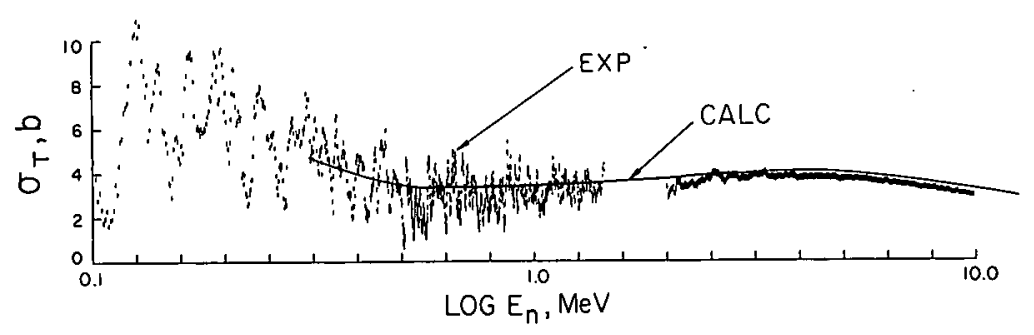

113-488 Rev. 1

Fig. 13. Measured and Calculated Total Cross Sections of Vanadium. Data for $E_{n}<1.5 \mathrm{MeV}$ from present work, for $E_{\mathrm{n}}>1.5 \mathrm{MeV}$ from the work of Galloway and Shrader. 36 


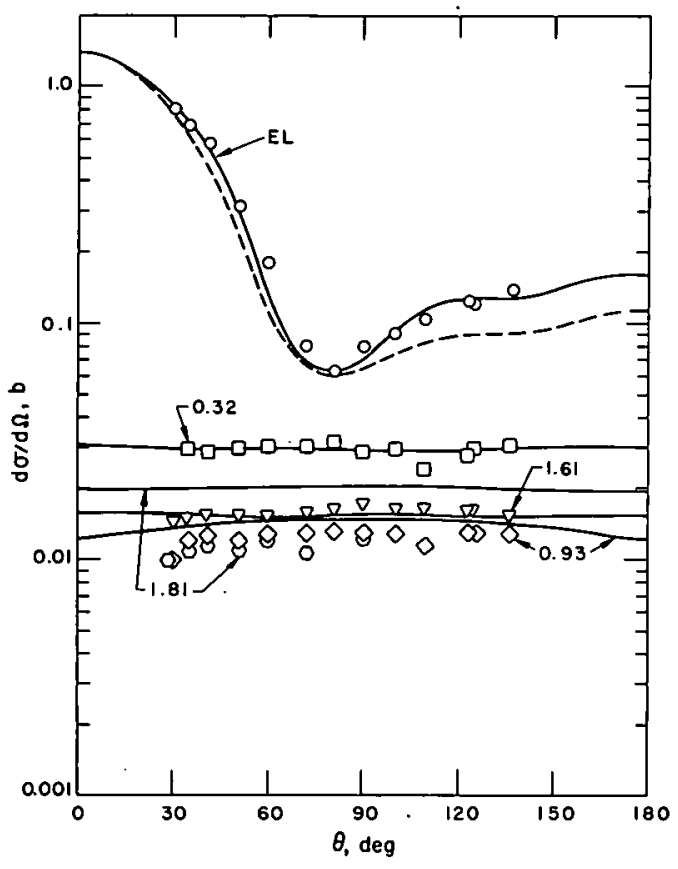

113-489 Rev. 1

Fig. 14. Calculated Elastic and Inelastic Distributions of Vanadium and the Results Measured by Towle 25 at $2.35 \mathrm{MeV}$. Solid curves were obtained using the "asymptotic potential" form of Eq. 3. For comparison, dashed curves are those calculated in Ref. 40.

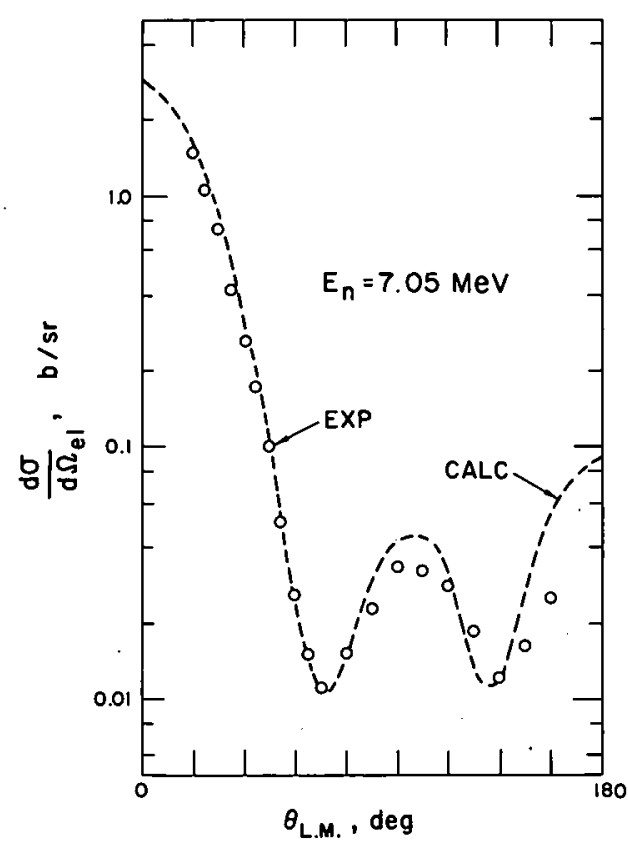

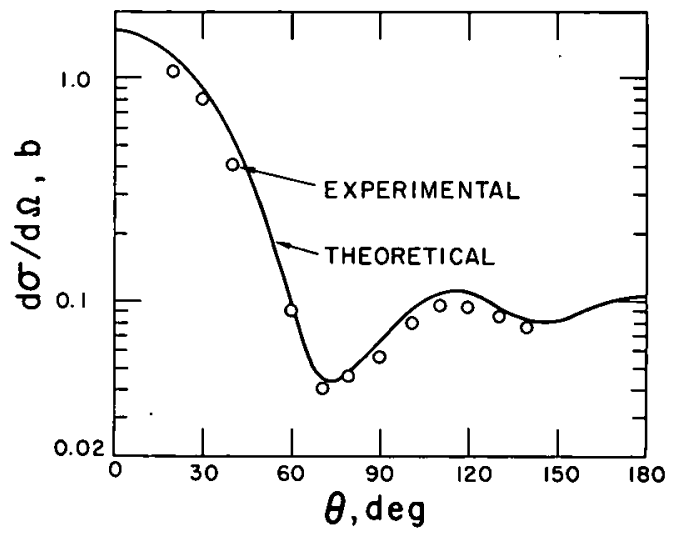

112-9573 Rev. 1

Fig. 15. Elastic Angular Distribution of Vanadium Calculated Using the "Asymptotic Potential" Form of Eq. 3, Compared with the Measured Values of Becker at $3.0 \mathrm{MeV}$. 37
Fig. 16

Elastic Scattering from Vanadium at $7.05 \mathrm{MeV}$. Points are measured values reported by Holmqvist and Wiedling. 38 The curve represents calculations using "asymptotic potential" of Eq. 3.

113-490 Rev. 1 


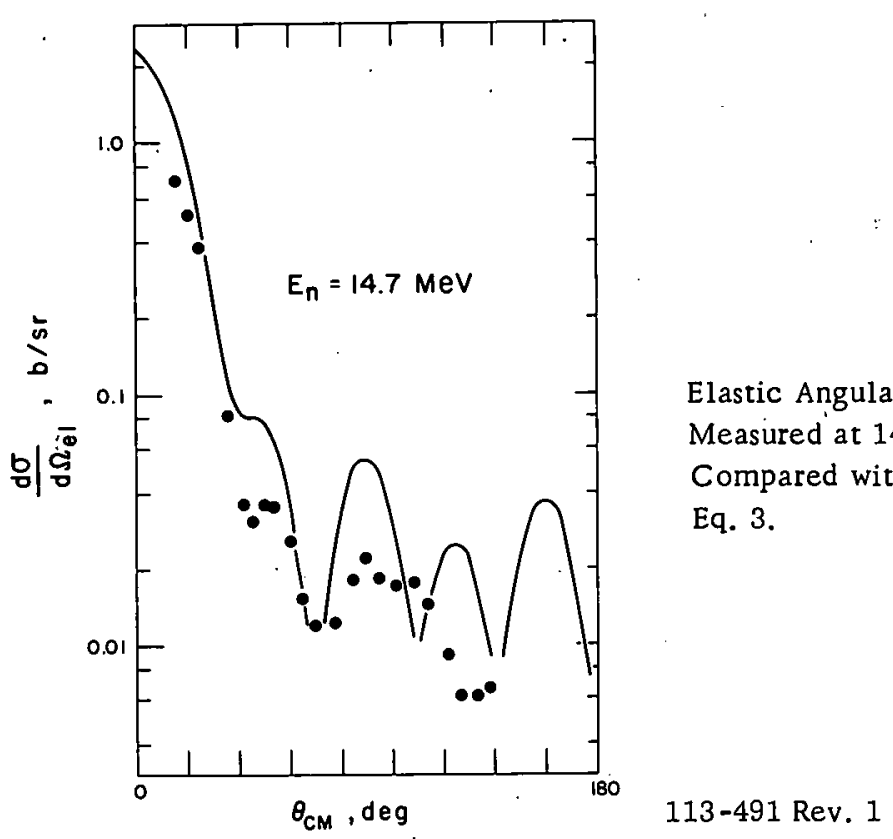

Fig. 17

Elastic Angular Distribution of Vanadium Measured at $14.7 \mathrm{MeV}$ by Western et al.., 39 Compared with Results Calculated from Eq. 3.

\section{B. Correlations and Fluctuations}

Correlations and fluctuations in the measured cross sections were examined with the intent of ascertaining the statistical properties of the observables and their physical significance. The interpretation took the forms of (a) a search for a quantitative measure of intermediate resonance structure, (b) an analysis based upon the premise of strongly overlapping resonances (Ericson fluctuations), $, 41,42$ and (c) a derivation of average level density from fluctuations in widths and spacings of compound nucleus resonances. 6,7

\section{Existence of Intermediate Resonance Structure}

Intermediate structure can be attributed to measured cross sections by qualitative inspection. Such procedures can be deceptive. ${ }^{43,44}$ A quantitative approach uses the function ${ }^{7,44}$

$$
C(R)=\frac{1}{N} \sum_{i=1}^{N}\left[\sigma\left(E_{i}\right)-\overline{\sigma\left(E_{i}\right)}\right]^{2}
$$

where

$$
\overline{\sigma\left(E_{i}\right)}=\frac{1}{R} \int_{E_{i}-R / 2}^{E_{i}+R / 2} \sigma(E) d E .
$$

$C(R)$ increases with $R$, asymptotically approaching a constant value for $R$ much larger than the average structure width of the fluctuating cross 
section. In the presence of two very different fluctuation widths $\left(\Gamma_{1}\right.$ and $\Gamma_{2}$, where $\left.\Gamma_{1}<<\Gamma_{2}\right), C(R)$ increases with $R$, approaching an initial plateau value for $\Gamma_{1}<R<\Gamma_{2}$ then rises to a second and final plateau for $R>\Gamma_{2}$. $\Gamma_{1}$ can be associated with the compound nucleus width $\left(\Gamma_{\mathrm{CN}}\right)$, and $\Gamma_{2}$ with a much larger intermediate resonance width $\left(\Gamma_{\text {IS }}\right)$. The experimental resolution used in the present total cross-section measurements was $\lesssim \Gamma_{\mathrm{CN}}$, sufficent to clearly indicate intermediate structure if present to an applicable extent. Figure 18 shows the function $C(R)$ calculated from the total crosssection values determined in the present experiment. The function rises to a single oscillatory plateau characteristic of finite range deviations (FRD) inherent in the limited experimental sample. ${ }^{45,46}$ This form of $C(R)$ is consistent with a single average width of a few keV. The intermediate structure qualitatively apparent in the total neutron cross sections (see Fig. 1) must, if valid, be of such a magnitude as to be masked by FRD and statistical fluctuations in the $C(R)$ distribution. $C(R)$ derived from the measured elastic scattering cross sections oscillated with such an amplitude as to preclude any reasonable assay of intermediate structure. Such behavior was not unexpected, in view of the small energy range of the sample and the precision of the measurements. In the present experimental context, the use of the $C(R)$ function for the assay of intermediate structure led to inconclusive results.

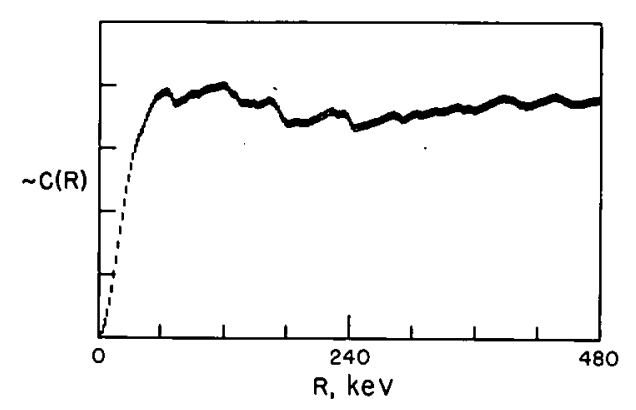

Fig. 18

Function $C(R)$, Eq. 5, Evaluated from the Measured Total Neutron Cross Sections of Vanadium for $0<\mathrm{R}<480 \mathrm{keV}$

\section{Limit of Strongly Overlapping Resonances, $\Gamma>>\mathrm{D}$}

Fluctuations have been observed in cross sections at energies at which the compound nucleus width is expected to greatly exceed the spacing $\left(\Gamma_{\mathrm{CN}} \gg \mathrm{D}_{\mathrm{CN}}\right) \cdot{ }^{7,17,47-49}$ Ericson performed a theoretical study of these fluctuations and related observable quantities to average compoundnucleus properties. 5,41,42 As used here, his interpretation is based upon a number of assumptions, primary of which are: (a) The reactions are purely statistical, (b) $\Gamma>\mathrm{D}$, (c) $\Gamma^{\mathrm{J}} \rightarrow \Gamma=$ a constant, (d) experimental resolutions $<\Gamma$, and (e) no single- or several-particle resonance effects exist. Qualitatively, $\Gamma / D=n / 2 \pi$, where $n$ is the number of effective exit channels. ${ }^{50}$ In the present experiments, $n$ was probably not $>2 \pi$; rather, $l=0$ strength functions ${ }^{19}$ indicated that $\Gamma / \mathrm{D}$ was more nearly unity. $\Gamma \mathrm{J} \rightarrow \Gamma=$ constant implies a number of available exit channels and a large nuclear moment of inertia, not particularly characteristic of the present experiments. The effect of several particle resonances may be appreciable over much of the 
measured energy interval (see Section IV.C below). Further, the experimental resolution used,in the present total cross-section measurements was $\lesssim \Gamma$ only in limited and lower-energy regions. Thus an interpretation of the present work in the framework of Ericson. fluctuations is open to considerable question. The approach is pursued here only in the spirit of a qualitative experimental assay.

Ericson derived the total cross - section correlation function ${ }^{5}$

$$
\begin{aligned}
& F(\epsilon)=\langle[\sigma(E+\epsilon)-\langle\sigma(E)\rangle][\sigma(E)-\langle\sigma(E)\rangle]\rangle \\
& F(\epsilon)=\frac{\Gamma^{2}}{\epsilon^{2}+\Gamma^{2}} \kappa \frac{D_{0}}{\pi \Gamma} \frac{\left(\pi \lambda^{2}\right)^{2}}{(2 \mathrm{i}+1)(2 \mathrm{I}+1)} \sum_{l}(2 \ell+1) \mathrm{T}_{\ell}^{2}:
\end{aligned}
$$

where $\kappa$ is a measure of the distribution of partial widths around the mean $\left(\sim 1.5\right.$ Porter-Thomas distribution $\left.{ }^{56}\right), \mathrm{DJ}^{\mathrm{J}}=\mathrm{D}_{0} /(2 \mathrm{~J}+1), \mathrm{T}_{l}=$ optical model transmission coefficient, and. $i$ and $I$ are.the spins of the incident and target particles, respectively. In the ratio form,

$$
C(\delta)=\frac{\langle\sigma(E+\delta) \cdot \sigma(E)\rangle}{\langle\sigma(E+\delta)\rangle\langle\sigma(E)\rangle}-1=\frac{1}{N}\left(1-Y^{2}\right) \frac{\Gamma^{2}}{\delta^{2}+\Gamma^{2}},
$$

where $\mathrm{N}=$ number of contributing independent channels and $\mathrm{Y}=$ the proportion of direct reactions. Equation. 7 is independent of $D_{0}$ and $\kappa .5,46$ Further,

$$
C(0)=\frac{1}{N}\left(1-\dot{Y}^{2}\right)
$$

and

$$
\left.F(0)=\kappa \frac{D_{0}}{\pi \Gamma} \frac{\left(\pi \lambda^{2}\right)^{2}}{(2 i+1)(2 I+1)} \sum_{\ell}(2 \ell+1) T_{l}^{2} \cdot\right\}
$$

In the following, Eqs. 7 and 8 are used to obtain $N$ and $\Gamma$ from the measured cross sections and (with the $T_{\ell}$ values. calculated using the "asymptotic potential" of Section IV.A) the value of $D_{0}$. Corrections are made for the effect of the finite experimental resolution. ${ }^{51,52}$. These corrections are not negligible, particularly at higher energies, where the assumptions of the theory become more applicable to the experiment.

The lower portion of Fig. 19 shows $C(\delta)$ calculated from the measured total cross sections. The function decreases rapidly from a maximum at $\delta=0$ to about half-magnitude, then slowly decreases in an oscillatory manner with increasing $\delta$. The slow decrease at large $\delta$. is characteristic of a direct-reaction (DR) modulation, and the oscillations are attributed to the finite sample size.45,46 The DR modulation (shape scattering) could be removed from the measured data using results of a 
suitable optical model calculation, but a model calculation of sufficient accuracy is difficult to achieve. As a phenomenological alternative, the DR component was determined by least-squares fitting the cubic

$$
\delta_{c a l}=a+b E+c E^{2}+d E^{3}(E \text { in } M e V)
$$

to the measured data. The order of the fit was such as to well describe the general energy dependence without unduly distorting the fluctuating structure. $\delta$ cal was subtracted from the measured cross sections, and a positive constant added to ensure nonnegative values. The resulting "fluctuating cross section," shown in Fig. 20 together with the directly measured values, was used in subsequent correlation analysis.

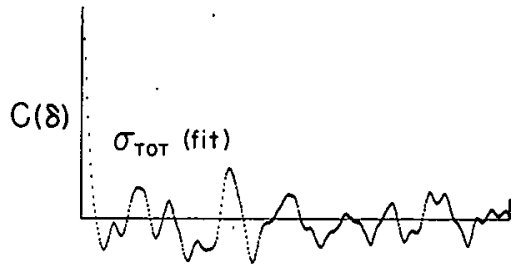

Fig. 19

$C(\delta)$ Evaluated from Total Neutron Cross Sections of Vanadium. Lower: $C(\delta)$, Eq. 7, evaluated from the total neutron cross sections of vanadium. The effects

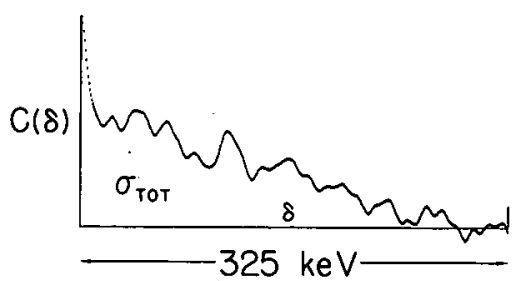
of direct reactions and the finite sample range are evident. Upper: $C(\delta)$ evaluated from the total neutron corrected for direct reactions.

113-499 Rev. 1

Fig. 20

Measured Total Neutron Cross Sections of Vanadium. Lower: measured total neutron cross sections of vanadium. Results are identical to those shown in Fig. 5. Upper: measured total cross-section values after correction for DR contributions in the manner described in the text.
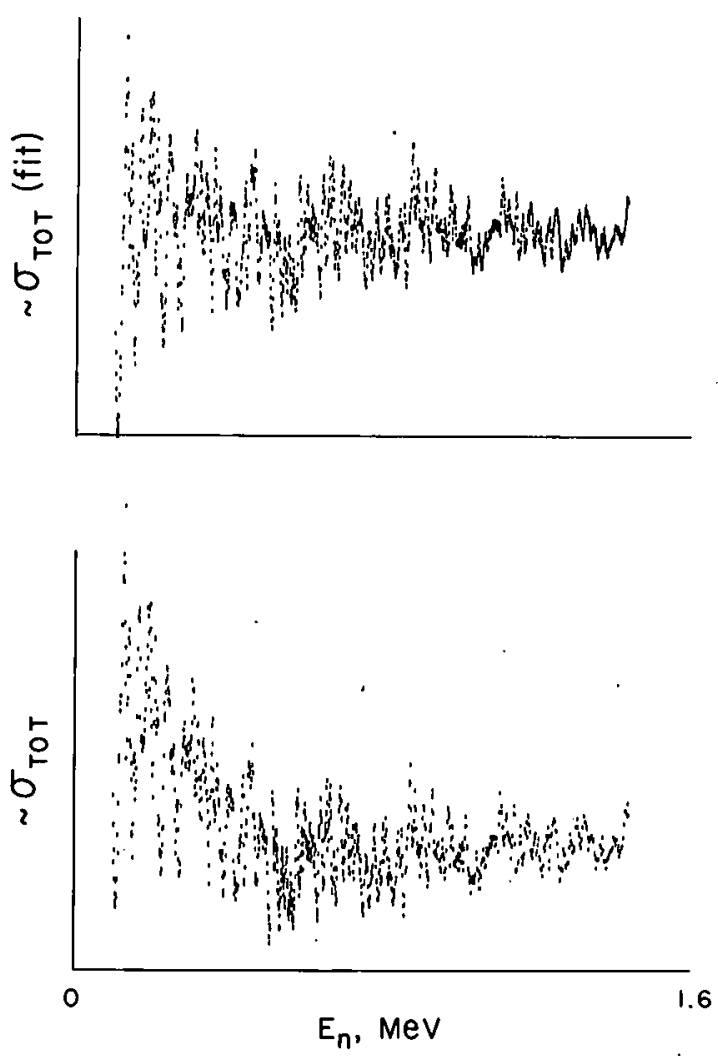
$F(\epsilon)$ and $C(\delta)$ (from Eqs. 6 and 7 ) were evaluated from the fluctuating total cross section over the entire measured energy interval and a number of subintervals thereof. Typical of the results is $C(\delta)$ calculated for the entire interval shown in the upper portion of Fig. 19. FRD oscillations remain evident and are of an $\mathrm{rms}$ magnitude consistent with the estimates of Hall. ${ }^{45,46}$ Neither in this example nor in any other $C(\delta)$ calculation was a significant deviation from a simple Lorentzian form noted as would be expected from appreciable intermediate resonance structure with a width $\Gamma_{I S}>\Gamma_{C N}$. Table I summarizes $C(0), F(0), \Gamma$, and $D_{0}$ values, derived from the correlation analysis of the total cross sections, carried out over the entire measured interval and subintervals thereof using Eqs. 6-8. Uncertainties in the calculated $C(0)$ and $F(0)$ values are large; FRD alone led to standard deviations of $30 \%$ or more. The $\Gamma$ values are remarkably consistent, and $D_{0}$ shows the expected energy dependence. However, $D_{0}$ tends to be an order of magnitude larger and $\Gamma$ a factor of two to three larger than indicated by level density formulas, by results of detailed low-energy resonance studies, and by the analysis of average cross sections at low energies. ${ }^{19,53,54}$ These discrepancies tend to compensate when the $l=0$ strength function is estimated, resulting in a value about one-fifth of that reported from detailed resonance studies and about onethird to one-half of that obtained from average low-energy cross sections. In view of the inappropriateness of the theory in the present experimental context, the results are probably remarkable for their agreement with values obtained by other and more proper methods and tend to indicate that such analyses of total neutron cross sections are not sensitive to these theoretical concepts and underlying premises.

TABLE I. Results of Correlation Analysis of Measured Total Cross Sections

\begin{tabular}{|c|c|c|c|c|c|c|c|c|c|c|}
\hline $\begin{array}{l}\text { Energy Interval, } \\
\text { MeV }\end{array}$ & $\begin{array}{l}0.1- \\
1.44\end{array}$ & $\begin{array}{l}0.1- \\
0.42\end{array}$ & $\begin{array}{l}0.21- \\
0.53\end{array}$ & $\begin{array}{l}0.32- \\
0.64\end{array}$ & $\begin{array}{l}0.43- \\
0.75\end{array}$ & $\begin{array}{l}0.53- \\
0.86\end{array}$ & $\begin{array}{l}0.64- \\
0.97\end{array}$ & $\begin{array}{l}0.75- \\
1.07\end{array}$ & $\begin{array}{l}0.85- \\
1.18\end{array}$ & $\begin{array}{l}0.97- \\
1.29\end{array}$ \\
\hline$C(0) \quad C(0)$ & 0.0378 & 0.101 & 0.0473 & 0.0353 & 0.0300 & 0.0234 & 0.0180 & 0.0152 & 0.0112 & 0.0081 \\
\hline$F(0), b^{2}$ & 1.103 & 3.03 & 1.384 & 0.957 & 0.848 & 0.647 & 0.546 & 0.439 & 0.346 & 0.235 \\
\hline$\Gamma, \mathrm{keV}$ & 4.0 & 5.1 & 4.4 & 3.7 & 3.8 & 3.8 & 3.9 & 4.0 & 3.2 & 3.0 \\
\hline $\mathrm{D}_{0}, \mathrm{keV}$ & 410 & 540 & 304 & 223 & 225 & 189 & 171 & 162 & 115 & 83 \\
\hline$D=D_{0}(2 \mathrm{~J}+1){ }^{a}$ & 49 & 65 & 36 & 26 & 27 & 23 & 20 & 19 & 13 & 10 \\
\hline$\Gamma /\left.0\right|_{0} \times 10^{a, b}$ & 0.96 & 1.5 & 2.0 & 2.0 & 1.8 & 2.0 & 2.2 & 2.2 & 2.4 & 2.8 \\
\hline
\end{tabular}

a Assuming $2 \mathrm{~J}+1 \cong 8$.

bAll values corrected to $\mathrm{l} \mathrm{eV}$.

As is evident from Eq. 7, correlations are damped as the number of exit channels increases and thus should become more pronounced as the number of reaction channels is restricted. Therefore, to obtain more definitive results, $C(\delta)$ was evaluated for the $B l$ coefficients of the elastic channel and for the excitation cross section of the inelastic channel $(\mathrm{Q}=-0.32 \mathrm{MeV})$. Unfortunately, these correlation functions all showed strong FRD oscillations because of the limited extent of the available 
sample. These oscillations and the experimental resolutions appreciably exceeding $\Gamma_{C N}$ precluded any reasonable interpretation of the $C(\delta)$ derived from either the elastic or inelastic scattering measurements.

The cross correlation function

$$
\mathrm{C}_{\mathrm{ab}}=\kappa \mathrm{G}_{\mathrm{ab}} \text {, }
$$

where

and

$$
\left.G_{a b}=\frac{\left\langle\sigma_{a} \cdot \sigma_{b}\right\rangle}{\left\langle\sigma_{a}\right\rangle \cdot\left\langle\sigma_{b}\right\rangle}-1\right\}
$$

is of interest. ${ }^{5,46} \mathrm{C}_{\mathrm{ab}}$ was determinedfor the $\mathrm{B}_{\ell}$ coefficients of the elastic cross section, the elastic and total cross sections, and the elastic and inelastic $(Q=-0.32 \mathrm{MeV})$ cross sections. The results are given in $\mathrm{Table}$ II. As expected, $B_{0}$ (approximately elastic cross section) was strongly correlated.with the total cross section (see Fig. 5). Between $B_{\ell}$ values, only the $B_{0}$ to $B_{1}$ correlation appears significantly larger than $F R D$ errors alone. This is in contrast to the concepts of Ericson, ${ }^{5}$ which indicate small correlations between even and odd $B_{\ell}$ coefficients. There was no significant numerical correlation between elastic and inelastic cross sections, though experimental uncertainties and the limited range of the samples might have masked the small effect that seems qualitatively present from a visual inspection of the respective cross sections.

\begin{tabular}{|c|c|c|}
\hline Processes & $\mathrm{G}_{\mathrm{ab}} \mathrm{a}, \mathrm{b}$ & $C_{a b} b^{b}$ \\
\hline $\mathrm{B}_{0}$ and $\sigma_{\mathrm{T}}$ & $\begin{array}{r}1.34 \times 10^{-2} \\
\pm 0.36 \times 10^{-2}\end{array}$ & 0.77 \\
\hline $\mathrm{B}_{0}$ and $\sigma_{\text {inel }}$ & $\begin{array}{r}0.18 \times 10^{-2} \\
\pm 0.40 \times 1.0^{-2}\end{array}$ & 0.088 \\
\hline$B_{0}$ and $B_{1}$ & $\begin{array}{r}1.4 \times 10^{-2} \\
\pm 0.60 \times 10^{-2}\end{array}$ & - \\
\hline$B_{0}$ and $B_{2}$ & $\begin{array}{l}-1.6 \times 10^{-2} \\
\pm 1.2 \times 10^{-2}\end{array}$ & - \\
\hline $\mathrm{B}_{1}$ and $\mathrm{B}_{2}$ & $\begin{array}{l}-1.5 \times 10^{-2} \\
\pm 2.1 \times 10^{-2}\end{array}$ & - \\
\hline
\end{tabular}

TABLE II. Cross-correlation Values

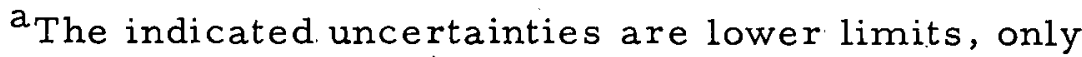
pertaining to errors associated with the finiteenergy range of the experimental samples. ${ }^{46}$

bDefined by Eq. 10. 
3. Cross-section Fluctuations and Distributions of Widths and Spacings

In this section, structure in the measured total cross sections of vanadium is considered in the context of fluctuations in compound nucleus resonance spacings and widths after the manner of Carlson and Barschall ${ }^{7}$ and of Agodi and Pappalardo. ${ }^{6}$ It is assumed that the experimental resolution appreciably exceeds the average compound nucleus width, and experimental averages are constructed to ensure the validity of the premise. Within these averages, the interference between potential and compound scattering is neglected and the former's smooth energy dependence is removed by the cubic fitting procedure described by Eq. 9. It is further assumed that the partial. widths are small compared to their spacings.

With the above premises, Carlson and Barschall derived the total cross section variance

$$
\mathrm{S}^{2} \text { (variance) }=\left(\pi \lambda^{2}\right)^{2} \sum_{\mathrm{J} \pi} \frac{\mathrm{g}^{2}}{\left\langle\mathrm{~N}_{\mathrm{J} \pi}\right\rangle}\left[\mathrm{k}_{\mathrm{w}} \sum_{\ell \mathrm{s}}\left(\mathrm{T}_{\ell \mathrm{s}}^{\mathrm{J}}\right)^{2}+\mathrm{k}_{\mathrm{n}}\left(\sum_{\ell \mathrm{s}} \mathrm{T}_{\ell \mathrm{s}}^{\mathrm{J}}\right)^{2}\right] \text {, }
$$

where $g=(2 J+1) /[2(2 I+1)],\left\langle N_{J \pi}\right\rangle$ is the average number of levels of a given $J \pi$ in the energy interval $\triangle$, and $T_{l s}^{J}$ are transmission coefficients. ${ }^{3}$ The derivation makes use of the definitions

$$
\begin{aligned}
& \mathrm{T}_{\ell s}^{\mathrm{J}}=2 \pi\left\langle\Gamma_{\ell s}^{\mathrm{J}}>/ \mathrm{D}_{\mathrm{J} \pi}\right. \\
& \mathrm{k}_{\mathrm{w}}=\operatorname{variance} \Gamma_{\ell \mathrm{s}}^{\mathrm{J}}\left\langle\Gamma_{\ell \mathrm{s}}^{\mathrm{J}}\right\rangle=\underset{\text {.widths }}{2 \text { for a Porter-Thomas distribution of }}
\end{aligned}
$$

and

$$
k_{n}=\operatorname{variance} N_{J \pi} /\left\langle N_{J \pi}\right\rangle:=\underset{\text { spacings } 57}{0.27 \text { for a Wigner distribution of }}
$$

Further, it is assumed that the distributions used in.calculating the variances are independent of quantum number and that the widths associated with differing quantum numbers and spacings for the same quantum number are uncorrelated. Some of these assumptions are not without question. ${ }^{4,58}$ If we separate the energy and spin dependence of the level density: in the manner of Gilbert and Cameron, ${ }^{54}\left\langle\mathrm{~N}_{\mathrm{J} \pi}\right\rangle / \Delta=\mathrm{G}(\mathrm{E}) \mathrm{H}(\mathrm{J} \pi)$, Eq. 11 reduces to

$$
s^{2} \Delta=\left(\pi \lambda^{2}\right)^{2} \frac{1}{G(E)} \sum_{J \pi} \frac{g^{2}}{H(J \pi)}\left[k_{w} \sum_{l s}\left(T_{l s}^{J}\right)^{2}+k_{n}\left(\sum_{\ell s} T_{l s}^{J}\right)^{2}\right]
$$


Using $T_{\ell s}^{J}$ obtained from the "asymptotic potential" of Eq. 3, $\mathrm{H}(\mathrm{J} \pi)$ from Ref. 54, and $\mathrm{S}^{2}$ determined from averages of measured values over the interval $\Delta$, we can obtain $G(E)$. Table III gives the results for several energy intervals of the measured total cross sections and a number of averaging increments, $\triangle$. In the table, the level density derived from the present experiments, $\rho_{\exp }=\mathrm{G}(\mathrm{E}) \cdot \mathrm{H}(\mathrm{J} \pi)$, is compared with that given by the formula of Gilbert and Cameron $\left(\rho_{\text {theo }}\right) .{ }^{54}$ For averaging increments $\Delta \gg \Gamma$ (i.e., $\Delta \gtrsim 25 \mathrm{keV}$ ), the Pexp values obtained from the experiments are within a factor of two of those given by.the density formula and are similar to values deduced from resonance studies at low energies. ${ }^{19}$ The results were not particularly sensitive to the distributions of widths and spacings used in evaluating $k_{\mathrm{w}}$ and $\mathrm{k}_{\mathrm{n}}$. Assuming exponential distributions $\left(k_{\mathrm{w}}=k_{\mathrm{n}}=1\right)$, or even neglecting width effects $\left(\mathrm{k}_{\mathrm{W}}=0, \mathrm{k}_{\mathrm{n}}=1\right)$, the results were similar to those obtained with Porter-Thomas and Wigner distributions. The $\left(\mathrm{k}_{\mathrm{w}}=0, \mathrm{k}_{\mathrm{n}}=1\right)$ assumption was that used by Agodi and Pappalardo. ${ }^{6}$ Within the experimental uncertainties of $30-50 \%$, the observed structure of the total cross section is consistent with the interpretation based upon recognized distributions of compound nucleus resonance widths and spacings. The distribution in spacings is a dominant factor, though the results are not sensitive to the detailed form of the distribution function.

TABLE III. Comparison of Experimentally and Theoretically Derived Level Densities

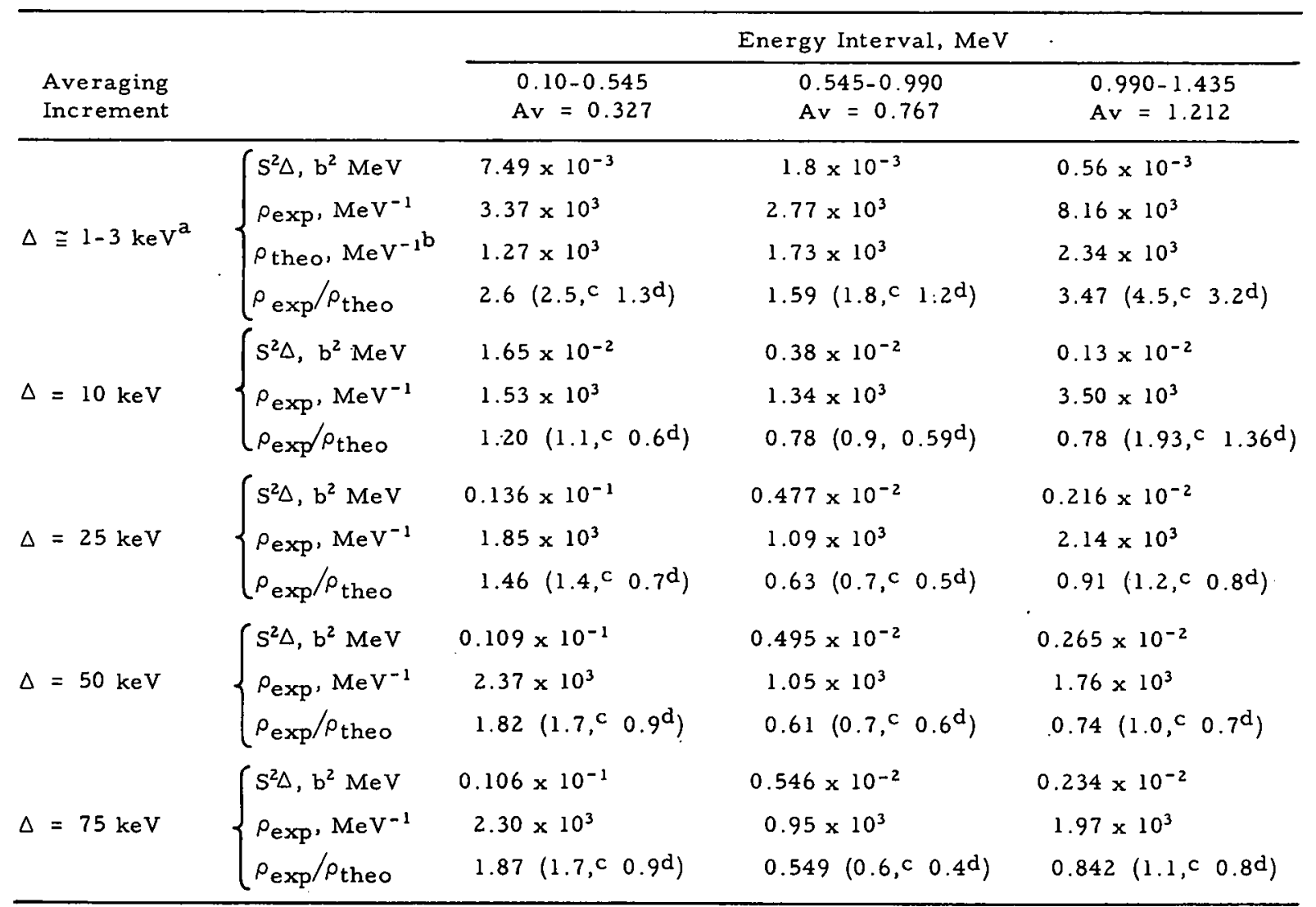

${ }^{a}$ Raw experimental data, resolution as per Section III.A, $\gtrsim 1 \mathrm{keV}$.

b Calculated from form of Gilbert and Cameron. ${ }^{54}$

${ }^{c}$ Ratio assuming exponential distribution of widths and spacings $\left(k_{w}=1, k_{n}=1\right)$.

dRatio assuming only exponential spacing distribution $\left(k_{w}=0, k_{n}=1\right)$. 
The expression $2 \pi\langle\Gamma / D\rangle=T$ used in deriving Eq. 11 is known to be invalid when $T$ approaches unity as it tends to in the present experiments. Considering resonance interference and $\underline{S}$ matrix unitarity, Moldauer has derived the relation $2 \pi\langle\Gamma / D\rangle=l_{n}[\bar{l} /(1-T)]$, valid for large $T .59$ The use of this relation increased the level densities derived from experiment ( $\rho_{\text {exp }}$ of Table III) by about a factor of three, to values appreciably larger than indicated by either level-density formulas or detailed resonance measurements. Such differences may, in part, be due to inappropriateness of Eq. 12 in the present experimental context. The equation is valid only for nonoverlapping resonances, and the Bethe randomness hypothesis ${ }^{6,55}$ is invoked to extend the validity to situations where partial widths alone are small compared to spacings. It is not clear that such an extrapolation is valid in the present experimental context.

C. The Intermediate Optical Model

The intermediate structure quantitatively evident in the energyaveraged total, elastic, and inelastic cross sections of vanadium can be characterized by a width, $\Gamma_{I S}$, and a spacing, $D_{I S}$, large compared to that of the compound nucleus and small relative to that of the single-particle or diffraction "giant resonances" (i.e., $\Gamma_{\mathrm{CN}}<\Gamma_{\mathrm{IS}}<\Gamma_{\mathrm{SP}}, \mathrm{D}_{\mathrm{CN}}<\mathrm{D}_{\text {IS }}<\mathrm{D}_{\mathrm{SP}}$ ). The observed structure in elastic and inelastic scattering processes ap! peared correlated in scattering angle (see Figs. 7 and 21).

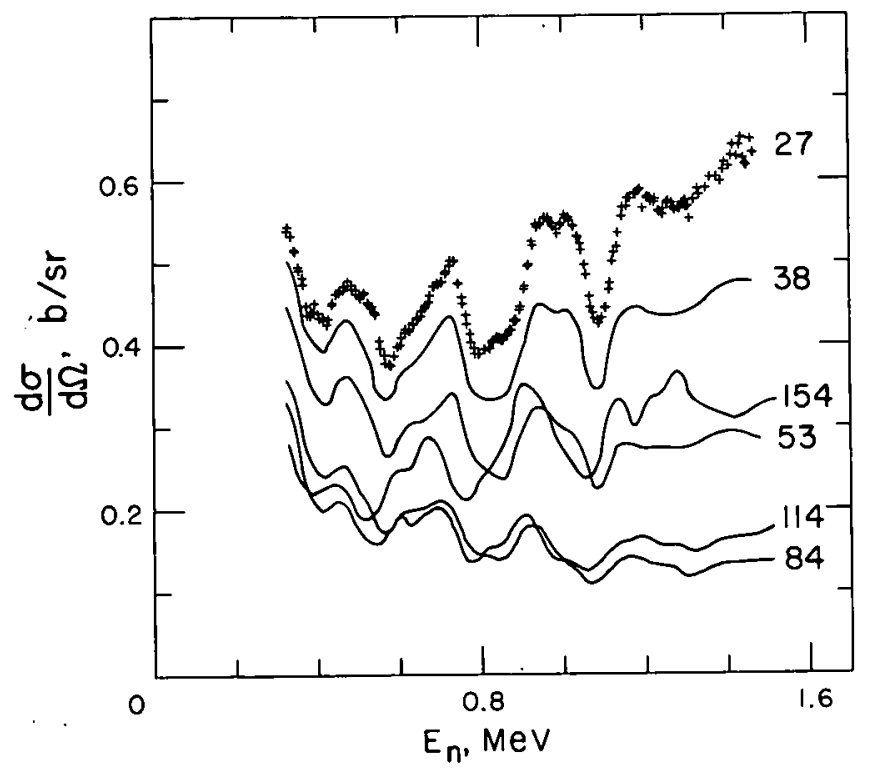

Fig. 21

Differential Elastic Neutron Scattering Cross Sections of Vanadium Observed at Laboratory Angles of $27-154^{\circ}$. The curves are derived from the measured values expressed in the form of Eq. 1. The results have been aver aged over a $50-\mathrm{MeV}$ energy interval to smooth local fluctuations. Actual measured values are indicated at $27^{\circ}$.

Structure of the type observed here has been interpreted in terms of "doorway state" processess."1,60,61 Many such interpretations tend to be qualitative comparisons with experiment. The "doorway state" has been quantitatively studied in the context of CN fine structure by Lejeune and Mahanx ${ }^{69}$ and by Takeuchi $^{8}$ and can be related to the properties of nuclear 
structure in the manner of Lemmer and Shakin, for example. ${ }^{70}$ Lipperheide,9,64 Feshbach et al., ${ }^{1}$ and Takeuchi $\mathbf{i}^{8}$ have suggested an "intermediate optical model." The concept of an intermediate optical model has the advantages of simplicity of application and of avoiding excessive demands on experimental resolution. This concept is used in the present interpretation, the theoretical aspects of which follow Ref. 71 .

Restricting the interpretation to the two open channels of $s$-wave elastic and inelastic scattering and assuming well-isolated doorway states and no direct reaction, the energy-averaged $T$-matrix for two open channels is 8,71

$$
\left\langle\mathrm{T}_{\mathrm{fi}}\right\rangle=\mathrm{T}_{\mathrm{fi}}^{\mathrm{p}}+2 \pi \sum_{\mathrm{s}} \mathrm{S}_{\mathrm{ff}}^{\mathrm{p}} \frac{\left\langle\chi_{\mathrm{f}}^{(+)} \mathrm{PHd} \Phi_{\mathrm{s}}\right\rangle\left\langle\phi_{\mathrm{s}} \mathrm{dHP}_{\mathrm{i}}{ }^{(+)}\right\rangle}{E-\mathrm{E}_{\mathrm{s}}-\Delta_{\mathrm{s}}+\frac{i}{2}\left(\Gamma_{\mathrm{s}}^{\downarrow}+\Gamma_{\mathrm{s}}^{\uparrow}\right)},
$$

where

$\mathrm{T}^{\mathrm{P}}$ and $\mathrm{S}^{\mathrm{P}}$ are potential scattering $\mathrm{T}$ - and $\mathrm{S}$-matrices,

$\chi^{(+)}$is the distorted wave satisfying outgoing-wave boundary conditions,

$E_{S}$ is the energy of the sth doorway state,

$\Gamma_{\mathrm{S}}^{\downarrow}$ is the decay width of the sth doorway to the $\mathrm{CN}$,

and

$\Gamma_{\mathrm{S}}^{\uparrow}$ is the decay width to the continuum.

The width $\Gamma_{\mathrm{S}}^{\uparrow}=\Gamma_{\mathbf{s}_{1}}^{\uparrow}+\Gamma_{\mathrm{s}_{2}}^{\uparrow}$, where $\Gamma_{\mathrm{s}_{1}}^{\uparrow}$ and $\Gamma_{\mathrm{S}_{2}}^{\uparrow}$ are elastic and inelastic contributions, respectively, and $P$ and $d$ are projection operators to the open channel and the doorway, respectively.

The wave equation for complex potential interactions in the presence of doorways is $1,8,9,62-64$

$$
\left[P H P-\sum_{S} f_{S}(E) \cdot P H d \phi_{S}\right\rangle\left\langle\phi_{S} d H P-E\right] \chi=0,
$$

where

$$
f(E)=1 /\left(E-E_{s}+i \Gamma_{s}^{\dagger} / 2\right)
$$

From this complex potential equation, a $\mathrm{T}$-matrix, $\mathrm{T}^{\mathrm{cP}}$, can in principle be derived equivalent to the energy-averaged $\mathrm{T}$-matrix of Eq. 13, and the total, absorption, elastic, and inelastic cross sections are obtained. ${ }^{71}$ The total cross section is proportional to the $T$-matrix and is given by ${ }^{8,9,64}$ 


$$
\left\langle\sigma_{\mathrm{c}}^{\mathrm{TOT}}\right\rangle=2 \pi \cdot \lambda_{\mathrm{c}}^{2}\left(-\operatorname{Im} \mathrm{T}_{\mathrm{cc}}^{\mathrm{cp}}\right) \text {. }
$$

The other cross sections follow from statistical considerations. The statistical properties of the $T$-matrix, $T=T^{D R}+T^{C N}$, have been extensively studied in the absence of a doorway. ${ }^{4,66}$ In the presence of a doorway, the exact $\mathrm{T}$-matrix can be written $\mathrm{T}=\mathrm{T}^{\mathrm{POT}}+\mathrm{T}^{\mathrm{d}}+\mathrm{T} \mathrm{T}^{1}{ }^{1} \mathrm{~T}^{\mathrm{q}}$ has the same structure as $\mathrm{T}^{\mathrm{CN}}$, and $\mathrm{T}^{\mathrm{d}}$ is assumed constant over an energy-averaging increment. Therefore $\mathrm{T}^{\mathrm{POT}}+\mathrm{T}^{\mathrm{d}}$ can be associated with $\mathrm{T}^{\mathrm{DR}}$, and $\mathrm{T}^{\mathrm{q}}$ with ${ }_{T} \mathrm{CN}$. With these associations, the energy-averaged cross sections are given by ${ }^{71}$

$$
\left\langle\sigma_{\mathrm{cc}}\right\rangle=\sigma_{\mathrm{cc}}^{\mathrm{opt}}+\sigma_{\mathrm{cc^{ \prime }}}^{\mathrm{fl}}
$$

where

$$
\begin{aligned}
& \sigma_{\mathrm{CC}^{\prime}}^{o p t}=\pi \lambda_{\mathrm{C}^{2}}^{2}\left|\mathrm{~T}_{\mathrm{cc^{ \prime }}}^{\mathrm{cp}}\right|^{2}, \\
& \sigma_{\mathrm{CC^{ \prime }}}^{\mathrm{fl}}=\pi \lambda_{\mathrm{C}}^{2} \frac{\mathrm{T}_{\mathrm{C}}^{\mathrm{CN}} \mathrm{T}_{\mathrm{C}^{\prime}}^{\mathrm{CN}}}{\sum_{\mathrm{C}^{\prime \prime}} T_{\mathrm{C}^{\prime \prime}}^{C N}},
\end{aligned}
$$

and

$$
\mathrm{T}_{\mathrm{c}}^{\mathrm{CN}}=1-\sum_{\mathrm{c}^{\prime \prime}}\left|\delta_{\mathrm{c}^{\prime \prime}}-\mathrm{i} \mathrm{T}_{\mathrm{cc}}^{\mathrm{cp}}\right|^{2}
$$

In practice, the calculation of these quantities from the coupled complexpotential wave equation, Eq. 14, is difficult, and a simplified method is sought. For this purpose, the cross section is obtained from the following decoupled equations:

$$
\left[P_{1} H P_{1}-\sum_{S} F_{S 1}(E) P_{1} H d \phi_{S}\right\rangle\left\langle\phi_{S} d H P_{1}-E\right] \chi_{1}=0,
$$

and

$$
\left[\mathrm{P}_{2} \mathrm{HP}_{2}-\sum_{\mathrm{S}} \mathrm{F}_{\mathrm{S} 2}(\mathrm{E}) \mathrm{P}_{2} \mathrm{Hd} \phi_{\mathrm{S}}\right\rangle\left\langle\phi_{\mathrm{S}} \mathrm{dHP} \mathrm{P}_{2}-\mathrm{E}\right] \chi_{2}=0,
$$

where $P_{1}$ and $P_{2}$ are projection operators for elastic and inelastic channels, respectively, and

$$
F_{s i}(E)=\frac{1}{E-E_{S}+\frac{i}{2}\left(\Gamma_{S}^{j}+\Gamma_{S}^{\uparrow}\right)_{j \neq i}} .
$$


The cross sections calculated from Eq. 17 can be compared with the energyaveraged cross sections of Eqs. 15 and 16.

The diagonal elements of the $T$-matrix derived from the decoupled Eqs. 17 are equivalent to thos ef $T^{\mathrm{cp}}$ obtained from the coupled. Eq. 14, and the total cross section obtained from Eqs. 17 is equivalent to the energyaveraged total cross section. $\sigma_{11}^{\mathrm{opt}}$ of Eq. 16 is equal to that obtained from Eqs. 17, but the $\sigma_{12}^{\text {opt }}$ of Eq. 17 is zero. Assuming $\Gamma_{\mathrm{s} 1}^{\uparrow}$ and $\Gamma_{\mathrm{s} 2}^{\uparrow}$ are equal, we can use transmission coefficients following from the decoupled equations to simply express elastic and inelastic cross sections in the following form: $:^{71}$

$$
\left\langle\sigma^{\mathrm{el}}\right\rangle=\left\langle\sigma_{\mathrm{OMHF}}^{\mathrm{el}}\right\rangle-\frac{\Gamma_{S 1}^{\uparrow}}{\Gamma_{\mathrm{S} 2}} \sigma_{11}^{\mathrm{HF}}
$$

and

$$
\left\langle\sigma^{\text {inel }}\right\rangle=\frac{\Gamma_{S}^{\downarrow}}{\Gamma_{S}^{\downarrow}+\Gamma_{S l}^{\downarrow}} \frac{\Gamma^{\uparrow}}{\Gamma_{S}^{\uparrow}+\Gamma_{S}^{\downarrow}} \sigma_{O M H F}^{\text {inel }} .
$$

Both of the above expressions are the familiar optical-model HauserFeshbach results modified by rather simple correction factors dependent on doorway-state widths.

Equations 17 have been interpreted as conventional optical-model equations, modified only by the presence of the energy-dependent factor $\mathrm{F}_{\mathrm{Si}}(\mathrm{E}) \mathrm{r}^{71}$ In the present analysis, an optical potential of the following form was chosen:

$$
\mathrm{V}^{\mathrm{opt}}=-\overline{\mathrm{V}}+\mathrm{i} \overline{\mathrm{W}}+\overline{\mathrm{V}}_{\mathrm{s} 0 \underline{\underline{l}}} \cdot \underline{\mathrm{s}}+\mathrm{F}_{1}+\mathrm{i} \mathrm{F}_{2} \text {, }
$$

$$
\}
$$

and

$$
F_{2}=\sum_{S} S_{S} \frac{-\Gamma_{S / 2}^{\downarrow}}{\left(E-E_{S}\right)^{2}+\left(\frac{\Gamma_{S}^{\downarrow}}{2}+\frac{\Gamma_{S}^{\dagger}}{4}\right)^{2}},
$$

$S_{S}$ being the strength of interaction with the $\mathbf{s}$ doorway. The imaginary component, W, of Eq. 20 is due to reactions not proceeding through explicitly identified doorway states. 
The potentials $\bar{V}, \bar{W}$, and $V_{\text {so }}$ were assumed to follow the spacial distributions of the phenomenological potential given in Eq. 3. The doorways are classified as the $\mathrm{J}^{\pi}$ each with associated strengths, resonant energies, $E_{S}$, and widths, $\Gamma \downarrow$. When averaged over energies large compared to those characteristic of the doorways, $F_{1} \rightarrow 0, F_{2} \rightarrow$ constant, and Eq. 20 reduces to the "conventional" optical potential. ${ }^{8}$ Other aspects of the intermediate potential, such as nonlocality and energy dependence, have been extensively discussed. 8,9

General application of Eq. 20 to the interpretation of experiment leads to the adjustment of a large number of parameters descriptive of each of a number of contributing doorway states. This would be true, for example, of charged-particle reactions at relatively high energies involving a number of angular momenta. ${ }^{65}$ The number of parameters. is sharply reduced in the consideration of the present experiments at incident energies $<1.0 \mathrm{MeV}$. $\ell=0$ processes are predominant with possible doorway configurations of $3^{-}$and $4^{-}$. Only the former can contribute to the inelastic channel $(Q=$ $-0.32 \mathrm{MeV}, l=0)$. The present interpretation is further simplified by assuming that the reactions are spin-independent and that possible doorway states do not appreciably overlap. Both assumptions are qualitative approximations, but they do permit a simple interpretation of the measured. results. in terms of the above intermediate model.

The procedure followed was to select, by inspection, average $\bar{V}$ and $\bar{W}$ values and a limited number of doorways characterized by $E_{S}$, $\Gamma_{S}^{\downarrow}$, and a strength, $\mathrm{S}_{\mathrm{S}}$, and calculate Vopt from Eq. 20 for: comparison with the real" and imaginary portions of the potential derived from a detailed fit to the measured elastic distributions (see Fig.10). Commensurate with the assumption of isolated doorways and the known compound nucleus structure, the number of doorway states considered was restricted to $\leqslant 5 / \mathrm{MeV}$. Doorway parameters were suitably adjusted where indicated; and the above procedure was repeated until a reasonable agreement was achieved between Vopt and the experimentally derived potential. The "intermediate" potential was then used to calculate elastic and inelastic distributions for direct comparison with the measured values. Rather good results were obtained after three or four iterations. Typical are the comparisons of real and imaginary portions of $\mathrm{V}^{\mathrm{opt}}$ and the experimentally derived potential shown in Fig. 22, and the elastic and inelastic results as measured and as calculated from $V^{\text {Opt }}$ shown in Fig. 23. The doorway parameters derived from these fitting procedures, assuming $\Gamma_{\mathrm{S} 1}^{\dagger}=\Gamma_{\mathrm{S} 2}^{\dagger}=\Gamma_{\mathrm{S}}^{\uparrow} / 2$, are given in $\mathrm{T}$ able IV. To avoid an upper-energy "end effect," three additional doorways were postulated and distributed uniformly between 1.1 and $1.6 \mathrm{MeV}$. Their effect on the calculated results below $\sim 0.8 \mathrm{MeV}$ was small. No additional doorway states were assumed below the minimum experimental observed energy of $\sim 0.3 \mathrm{MeV}$. 

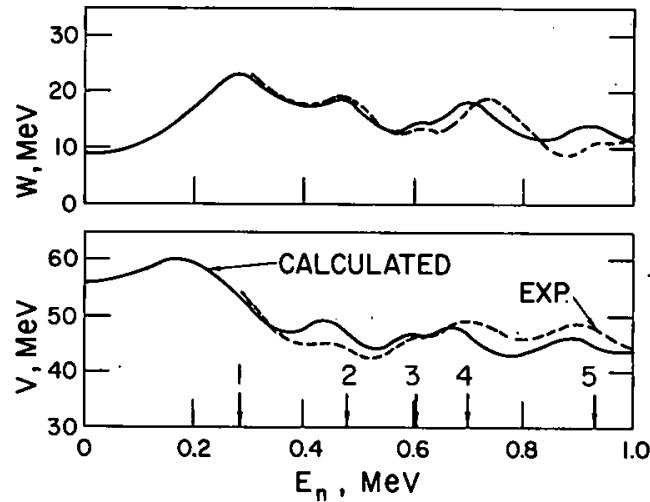

Fig. 23

Comparison of Experimental Elastic Scattering Angular Distributions and Inelastic Cross Sections, EXP., of Vanadium with Those Calculated from the Intermediate Optical Potential and Statistical Theory, CAL.
Fig. 22

Real (V) and Imaginary ( $W$ ) Portions of the Intermediate Optical Potential (Eq. 20), Calculated, Compared with the Phenomenological Optical Potential Derived from a Fit to the Measured Elastic Scattering Distributions, EXP. Positions of doorway states are indicated by arrows referring to Table IV values.

$113-1872$
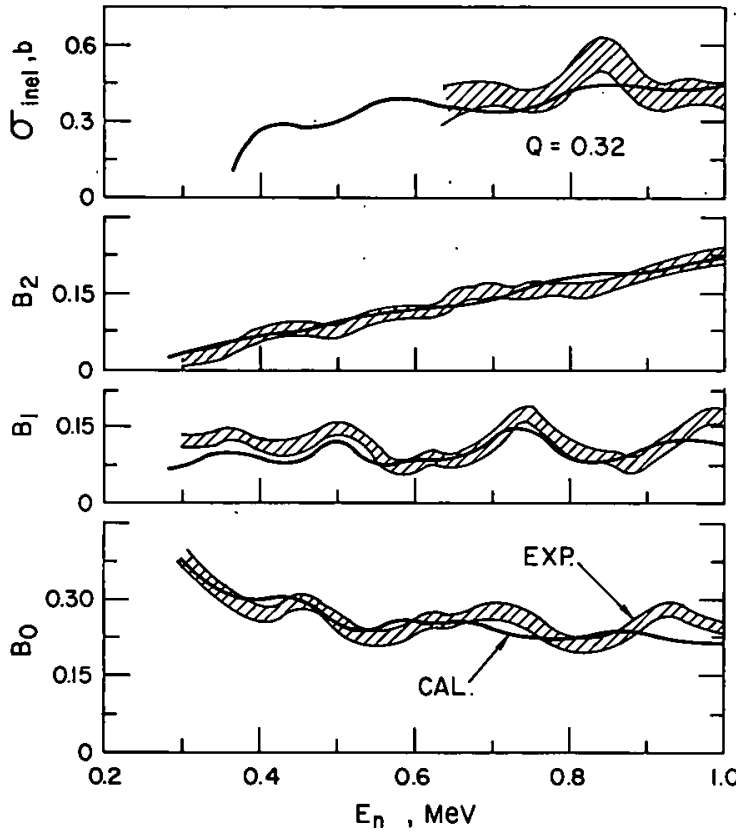

TABLE IV. Doorway Potential Parameters Used with Eq. $20^{a}$

\begin{tabular}{cccccc}
\hline $\begin{array}{l}\text { No. of } \\
\text { State }\end{array}$ & $E_{S}, \mathrm{MeV}$ & $\Gamma_{\mathrm{S}}^{\uparrow} / \Gamma_{\mathrm{S}}^{\dagger}$ & $\begin{array}{c}\Gamma_{\mathrm{S}}(\text { tot }), \\
\mathrm{MeV}\end{array}$ & $\begin{array}{c}\mathrm{T}_{0} \\
\text { Peak }\end{array}$ & $\begin{array}{c}\text { Strength, } \\
\mathrm{MeV}^{2}\end{array}$ \\
\hline 1 & 0.28 & 0.3 & 0.31 & 0.74 & 1.80 \\
2 & 0.48 & 2.02 & 0.135 & 0.88 & 0.70 \\
3 & 0.60 & 3.52 & 0.11 & 0.54 & 0.22 \\
4 & 0.70 & 1.99 & 0.17 & 0.89 & 0.99 \\
5 & 0.93 & 2.33 & 0.27 & 0.78 & 0.97 \\
\hline
\end{tabular}

a.Calculations used $\bar{V}=48.0 \mathrm{MeV}$ and $\bar{W}=6.5 \mathrm{MeV}$. 
$V^{\text {opt }}$ qualitatively describes the features of the experimentally derived potential (see Fig. 22) with the more serious differences at the higher incident energies, where $l \neq 0$ contributions are of increasing importance. Vopt also provides a similar qualitative description of the measured elastic angular distributions (see Fig. 23). The.structure of the $B_{1}$ coefficient is particularly well portrayed by calculations based upon vopt. The correlation between measured and calculated inelastic scattering is less satisfactory, but the former did not appreciably extend over much of the appropriate energy range of $\mathrm{V}^{\mathrm{opt}}$. From the doorway parameters (listed in Table IV) and $\mathrm{T}_{0}$ calculated at the peaks of the respective states, the ratios $\Gamma^{\uparrow} / \Gamma^{\downarrow}$ and the total doorway width $\Gamma=\Gamma^{\uparrow}+\Gamma^{\downarrow}$ can be evaluated, ${ }^{1,8,71}$ with the results given in Table IV. Though speculative, the quantities are: of magnitudes commensurate with the estimates of theory. ${ }^{1,8}$ Averaging $F_{1}$ and $F_{2}$ of Eq. 20 over energy intervals much greater than $\Gamma_{I S}$ or $D_{I S}$. leads to a vopt that reasonably describes the measured results averaged over a $200-\mathrm{keV}$ energy interval. However, the $s$-wave strength function calculated from $V$ opt for $E \rightarrow 0$ is approximately a factor of seven smaller than indicated by direct observation ${ }^{19}$ or systematics. ${ }^{57}$ This may reflect the presence of doorways near zero or negative energy not identified by the present experiments.

The above interpretation is consistent with the presently observed structure in the total and elastic scattering cross. sections of vanadium. This interpretation is reasonably free of gross assumptions or the use of an unduly large number of parameters. It is a logical development of the general optical potential. The interpretation provides qualitative knowledge of the properties of doorway resonances without detailed understanding of $\mathrm{CN}$ resonance structure. Indeed, the method precludes a direct correlation between explicit $C N$ and doorway resonance parameters, in the manner of Monahan and Elwyn. ${ }^{67}$ 


\section{COMPARISON WITH ENDF-B}

Since a motivation of the present work was the provision of fastneutron data for reactor design, it is instructive to compare the present results with the pertinent contents of the Evaluated Nuclear Data File-B $\left(\right.$ ENDF -B). ${ }^{10,68}$

As indicated in Fig. 24, the total cross sections determined in the present work compare reasonably well with the ENDF-B values. The ENDF - B evaluation was finished before the completion of the present work and therefore does not show the recently measured detailed resonance behavior. At higher energies, the ENDF-B values are in good agreement with the work of Galloway and Shrader ${ }^{36}$ and extrapolate well during the uncertain interval from 1.5 to $2.0 \mathrm{MeV}$. Throughout the range $0.3-10.0 \mathrm{MeV}$, the ENDF-B values are closely followed by the total cross section calculated using the potential of Section IV.A, as shown in Fig. 25. Elastic scattering cross sections contained within the ENDF-B file are in a somewhat different format from that used in Section III.B. However, when transformed to the present base (format of Eq. 1), they are remarkably descriptive of the experimental values, as indicated in Fig. 26. Such differences as do exist are largely in regions of strong fluctuations. Figure 27 compares experimental and ENDF-B inelastic scattering values. The evaluation relied to an appreciable extent upon optical-model and statistical calculations, and these show the same tendency toward low cross sections for the excitation of the $320-\mathrm{keV}$ state, particularly near threshold, noted in Section IV.A. At higher energies, ENDF-B and experimental values reported by Towle are in good agreement. ${ }^{25}$

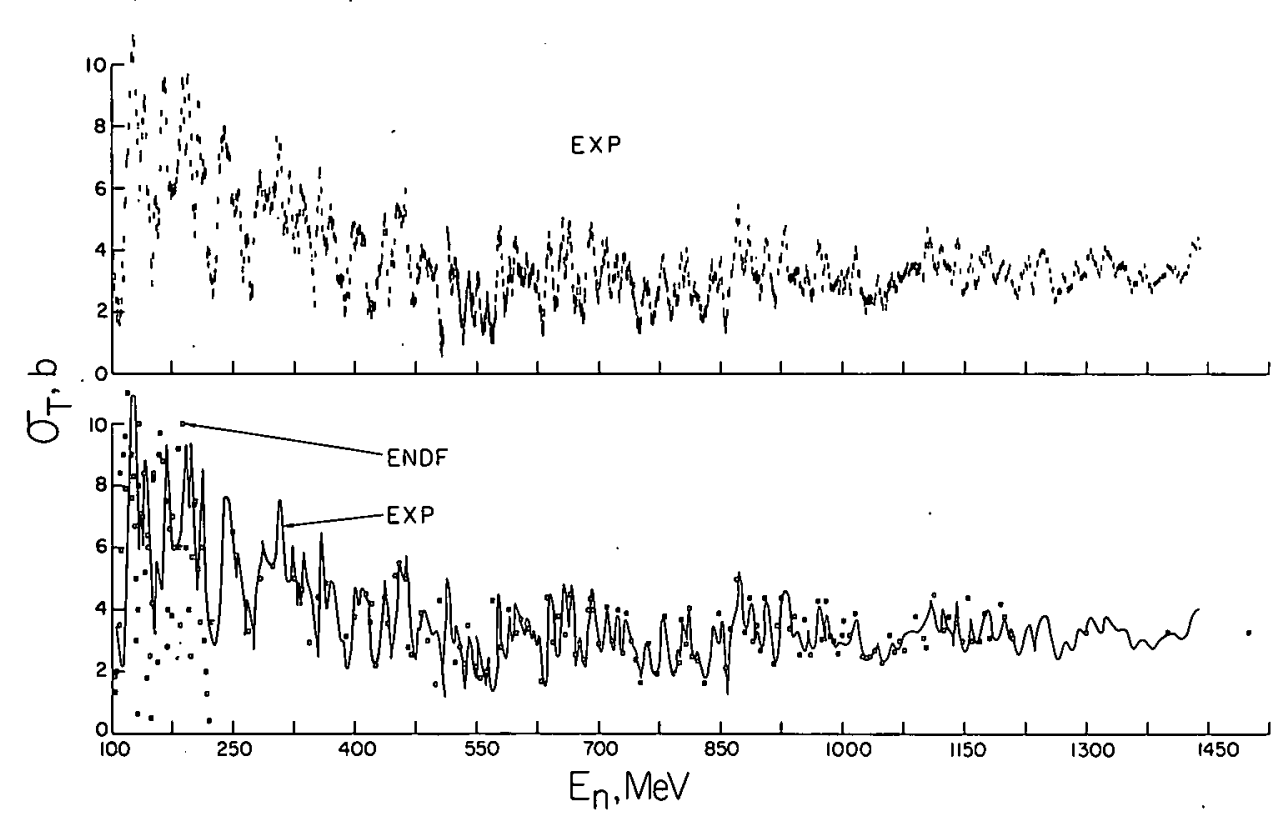

$112-9469$ Rev, 1

Fig. 24. Total Cross Sections of Vanadium Derived from Present Work (top) and ENDF-B Data ${ }^{10}$ (lower) 


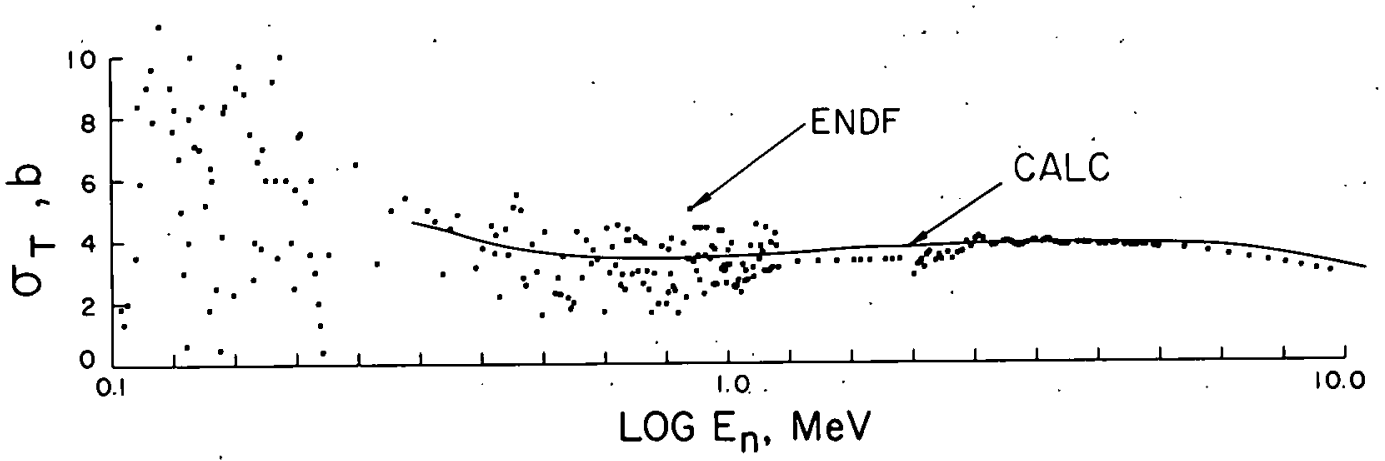

$113-492$

Fig. 25. Total Cross Sections, Calculated as Described in Section IV.A, Compared with ENDF-B Values

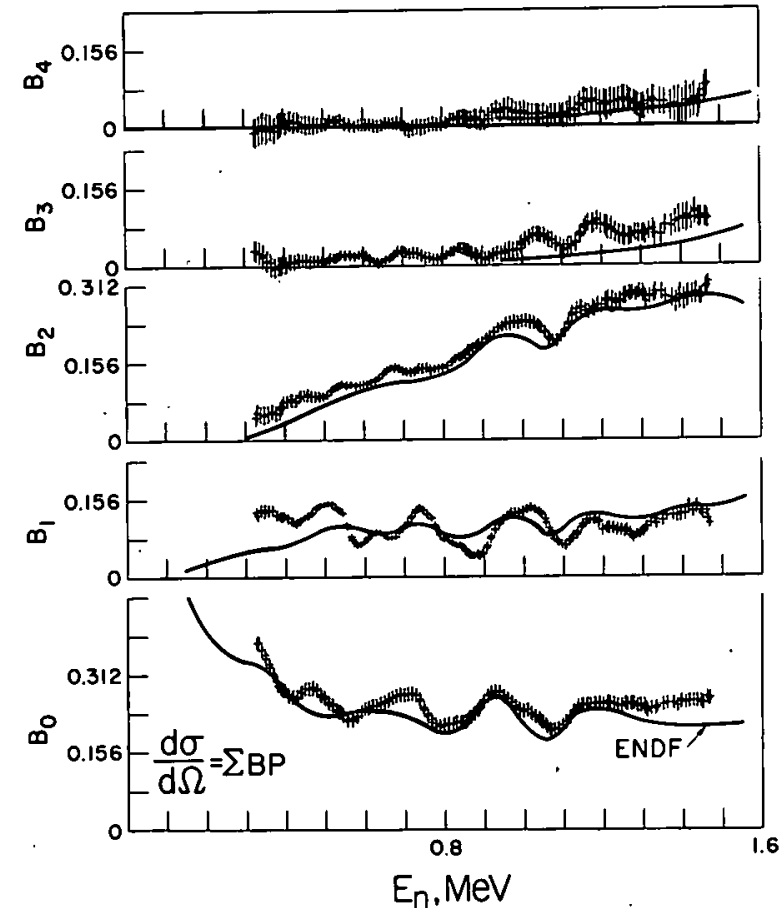

Fig. 27

Measured and ENDF-B Inelastic Excitation Cross Sections of Vanadium. Data below $1.5 \mathrm{MeV}$ (crosses) are from present work. Solid data circles are values reported by Towle. 25 Solid curves are ENDF-B values. Reaction $Q$ values are indicated.

113-496 Rev. 1
Fig. 26

Elastic Scattering Cross Sections of Vanadium Determined in the Present Work (data points) and the Values Given by ENDF-B (solid curves)

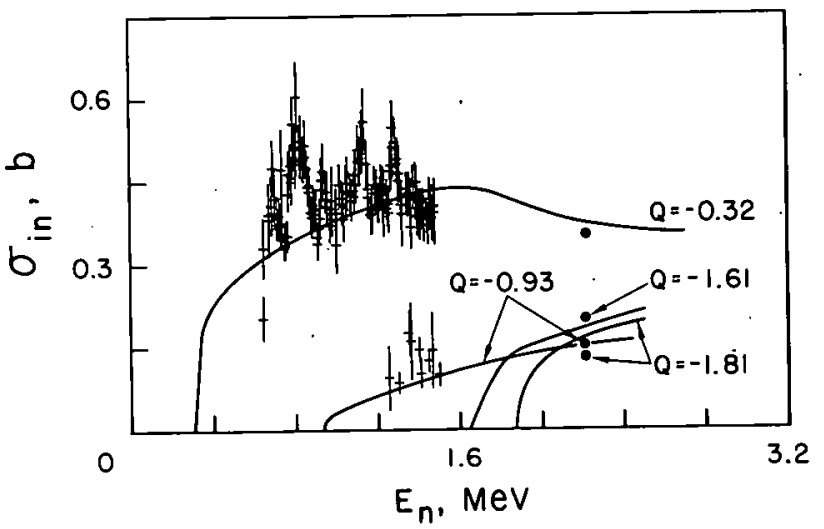


Generally, the ENDF-B file gives a reasonable description of the results of the present experiments, though the latter show more detailed resonance structure and result in somewhat larger inelastic cross sections. Whether these are critical matters may depend upon the use of the information in the design of reactor systems. The ENDF-B values at energies $>1.5 \mathrm{MeV}$ closely correspond to other reported measurements of total and scattering cross sections and to the predictions of the optical model and statistical calculations outlined in Section IV.A. 


\section{CONCLUDING REMARKS}

The observed total; elastic and inelastic scattering cross sections were characterized by both a fine and intermediate energy-dependent structure. Though of good resolution, the results did not provide a definition commensurate with compound-nucleus resonance analysis, and the interpretation was confined to the intermediate structure and the average energy dependence of the observed quantities.

A phenomenological optical-potential was shown descriptive of total and elastic scattering cross sections of vanadium over a wide energy range. Results of calculations based upon this potential and statistical theory were qualitatively descriptive of the observed inelastic scattering, but quantitatively deviated from measurement, particularly near the reaction thresholds. Consideration of alternative spin-parity assignments and/or the effects of resonance width fluctuations failed to enhance the agreement with the present experiments.

An attempt to use correlation analysis in a quantitative assay of the observed intermediate structure was inconclusive because of the effects of the finite sample range, the presence of direct reactions, and the uncertainties of the measurements.

The suitability of Ericson's concepts in the context of the present experiments is highly questionable. However, an interpretation based upon them led to reasonable strength functions, though the derived $\Gamma$ and $D$ were inconsistent with both experimental evidence and the basic premise ( $\Gamma \gg D)$.

An experimental interpretation of the observed structure in the total cross section based upon known compound-nucleus distributions in widths and spacings, assuming a nonoverlapping of partial resonance widths, resulted in compound-nucleus-level densities similar to those predicted by systematics and by extrapolation from detailed low-energy resonance studies. The result was not particularly sensitive to the form of the distributions of either $\Gamma$ or $D$, or even to the omission of the former.

The results of calculation using an intermediate optical potential, based upon the premise of a few isolated doorway states, were in qualitative agreement with the experimental elastic distributions and the phenomenological potential derived therefrom. This intermediate model used relatively few doorway parameters, and comparison with experiment led to parameter values of the magnitude expected from theoretical estimates. A wider application of the method may assist in determining some of the statistical properties of doorway states.

The latter two of the above interpretations of the intermediate structure appear physically applicable and result in qualitative agreement 
with experiment. The approaches are not unique, and other interpretations have led to similar qualitative successes. ${ }^{59,67}$ The various interpretations are not necessarily mutually exclusive and may each appreciably contribute to the physical reality.

The present work indicates that the evaluated vanadium data (ENDF-B) often used in reactor calculations is reasonably representative of microscopic measurement and should be suitable for many applied studies. As increasingly fine energy groups are used in macroscopic calculation, it may become desirable to refine the evaluated data to take more cognizance of the observed fine-resonance structure. Further, it may be desirable to revise the evaluated file to bring it into better agreement with the results of the present and other recent studies of the inelastic neutron-scattering cross sections of vanadium. Such revisions will be appreciable near the first inelastic threshold $(Q=-0.32 \mathrm{MeV})$. 


\section{APPENDIX}

\section{Tabulations of Measured Cross Sections}

Tables V-VIII are provided for the use of those individuals interested in numerical values of the experimental quantities, either for pure or applied reasons.

TABLE V. Observed Total Neutron Cross Sections of Vanadium

\begin{tabular}{|c|c|c|c|c|c|}
\hline$E_{n}, \mathrm{keV}$ & $\sigma_{\mathrm{T}}, \mathrm{b}$ & $\mathrm{d} \sigma_{\mathrm{T}}, \mathrm{b}$ & $E_{n}, k e V$ & $\sigma_{\mathrm{T}}, \mathrm{b}$ & $d \sigma_{T}, b$ \\
\hline 100.79 & 4.58 & .09 & 101.09 & 3.01 & .09 \\
\hline 102.89 & $2 . \times 2$ & .09 & 103.89 & 3.15 & .09 \\
\hline 104.89 & 2.39 & .09 & 100.89 & 1.78 & .09 \\
\hline 106.89 & 1.97 & .09 & 107.99 & 2.33 & .09 \\
\hline 108.99 & 1.65 & צ U & 109.99 & $1, y 1$ & .09 \\
\hline 110.99 & 1.97 & .09 & 111.99 & 2.36 & .09 \\
\hline 112.99 & 3.54 & .09 & $11 \mathrm{~s}, y 9$ & 3.07 & .09 \\
\hline 114.99 & 4.36 & צ' & 110.49 & 3.46 & .09 \\
\hline 116.99 & 5.61 & . & $11 \% .99$ & 6.18 & .09 \\
\hline 118.99 & 0.99 & .09 & 120.09 & 1.19 & .09 \\
\hline 121.09 & 8.11 & .US & $122 . \cup 9$ & 9.05 & .09 \\
\hline 123.09 & 9.04 & . & $1: 4.09$ & $10,<0$ & .09 \\
\hline 125.09 & 10.32 & .49 & 120.09 & 10.50 & .09 \\
\hline 127.09 & 10.18 & צy & 120.10 & 10.69 & .09 \\
\hline 129.10 & 12.19 & .05 & 130.10 & $9.1 \ddot{2}$ & .09 \\
\hline 131.09 & 0.40 & . US & 132.09 & 1.21 & .09 \\
\hline 133.09 & 3.04 & .09 & 134.09 & 5.54 & .09 \\
\hline 135.09 & 6.02 & .09 & 136.09 & 6.96 & .09 \\
\hline 137.09 & 7.13 & .09 & iso. ن & 7.10 & .09 \\
\hline 139.19 & 8.17 & .US & $14 U .19$ & 8.10 & .09 \\
\hline 141.19 & 0.93 & .09 & 142.19 & 8.27 & .09 \\
\hline 143.20 & 7.70 & .05 & 144.19 & 5.49 & .09 \\
\hline 145.19 & 5.07 & .09 & 140.19 & 5.87 & .09 \\
\hline 147.19 & 5.40 & . UY & 140.19 & 4.99 & .09 \\
\hline 149.19 & 3.61 &.$U S$ & $150: 19$ & 3.32 & .09 \\
\hline 151.19 & 2.94 & .09 & $1 b 2,19$ & 3.63 & .09 \\
\hline 153.19 & 5.13 & .09 & 154.19 & 5.58 & .09 \\
\hline 155.19 & 5.07 & .09 & 156.19 & 5.46 & .09 \\
\hline 157.19 & 4.74 & .09 & 153.19 & 4.47 & .09 \\
\hline 159.19 & 5.28 & .09 & 160.19 & 4.94 & .09 \\
\hline 161.19 & 6.05 & .09 & 162.19 & 1.88 & .09 \\
\hline 163.19 & 8.43 & צy & 164.19 & 9.24 & .09 \\
\hline 165.19 & 9.39 & .09 & 160.19 & 9.24 & .09 \\
\hline 167.19 & 9.49 & ن & 108.19 & 9.17 & .09 \\
\hline 169.29 & 8.17 & .09 & 170.29 & 6.63 & .09 \\
\hline 172.29 & $0 . \ddot{1} 1$ & .09 & 173.29 & 6.13 & .09 \\
\hline 174.29 & 5.97 & .49 & $17.2 .<9$ & 5.14 & .09 \\
\hline 176.29 & 6.04 & .US & 177.49 & 5.66 & .09 \\
\hline 178.49 & 5.89 & . ग9 & 278.49 & 6.46 & .09 \\
\hline 180.49 & 5.96 &.$U 9$ & 181.49 & $0 .<8$ & .09 \\
\hline
\end{tabular}


TABLE V (Contd.)

\begin{tabular}{|c|c|c|c|c|c|}
\hline$E_{n}, k e V$ & $\sigma_{\mathrm{T}}, \mathrm{b}$ & $\mathrm{d} \sigma_{\mathrm{T}}, \mathrm{b}$ & $E_{n}$, keV & $\sigma_{\mathrm{T}}, \mathrm{b}$ & $\mathrm{d} \sigma_{\mathrm{T}}$, \\
\hline 182.49 & 6.57 & . U9 & 185.49 & 6.03 & .09 \\
\hline 184.49 & 7.50 & .09 & 185.49 & 1.31 & .09 \\
\hline 186.49 & 7.93 & . 49 & $18 / .09$ & 8.49 & .09 \\
\hline 188.59 & 9.49 & .US & 189.59 & 9.29 & .09 \\
\hline 190.59 & 8.19 & .09 & 191.29 & 8.65 & .09 \\
\hline 192.59 & 7.17 & $.0 Y$ & 193.29 & 7.56 & .09 \\
\hline 194.59 & 9.47 & .09 & 190.59 & 9.61 & .09 \\
\hline 196.59 & 8.95 & .09 & $19 \%: 59$ & 8.59 & .09 \\
\hline 198.59 & 6.81 & .09 & 199.39 & 7.65 & .09 \\
\hline 200.59 & 6.38 & .09 & 201.29 & 5.58 & .09 \\
\hline 202.59 & 5.11 & .09 & 203.59 & 4.32 & .09 \\
\hline 204.59 & 5.41 & . UY & 205.29 & 6.98 & .09 \\
\hline 206.59 & 7.64 & .09 & $20 \% .29$ & 8.73 & .09 \\
\hline 208.59 & 8.69 & .09 & 209.29 & 7.55 & .09 \\
\hline 210.59 & 7.04 & .03 & 211.29 & 6.21 & .09 \\
\hline 212.59 & 6.39 & .09 & 213.29 & 6.04 & .09 \\
\hline 214.59 & 6.57 & .09 & 215.59 & 6.42 & .09 \\
\hline 216.59 & 6.53 & . U9 & 217.29 & 3.03 & .09 \\
\hline 218.59 & 4.02 & .09 & 219.59 & 3.24 & .09 \\
\hline 220.59 & 3.93 & .09 & 221.29 & 3.15 & .09 \\
\hline 222.59 & 3.05 & .09 & 225.29 & 2.49 & .09 \\
\hline 224.59 & 2.53 & .09 & פל.ל22 & 2.65 & .09 \\
\hline 226.59 & 3.48 & .09 & 2.27.27 & 3.29 & .09 \\
\hline 228.59 & 3.70 & .09 & 229.29 & 3.14 & .09 \\
\hline 230.59 & 4.79 & .49 & 231.39 & $5.0 \%$ & .09 \\
\hline 232.59 & 7.01 & .09 & 255.39 & 6.27 & .09 \\
\hline 234.59 & 6.70 & .09 & 235.39 & 7.25 & .09 \\
\hline 236.59 & 7.29 & .09 & و5. 231 & 7.01 & .09 \\
\hline 238.59 & 7.94 & .09 & 259.59 & 7.70 & .09 \\
\hline 240.59 & 7.89 & .09 & 241.59 & 7.38 & .09 \\
\hline 242.59 & 7.08 & .09 & 243.59 & 7.26 & .09 \\
\hline 244.59 & 7.47 & .09 & 245.29 & 7.02 & .09 \\
\hline 246.59 & 6.97 & .09 & 247.39 & 5.60 & .09 \\
\hline 248.59 & 5.65 & .09 & $248: 59$ & 5.74 & .09 \\
\hline 250.59 & 5.54 & .09 & 251.69 & 5,18 & .09 \\
\hline 252.69 & 5.21 & 9 ن & $2 b 3.69$ & 5.13 & .09 \\
\hline 254.69 & 5.33 & .09 & 253.09 & $2.0 \%$ & .09 \\
\hline 256.69 & 5.88 & .09 & 257.69 & 3.05 & .09 \\
\hline 258.69 & 5.16 & .09 & 259.09 & 4.12 & .09 \\
\hline 260.69 & 3.92 & .09 & 261.09 & 3.37 & .09 \\
\hline 262.69 & 3.06 & .09 & 265.70 & 3.07 & .09 \\
\hline 264.69 & 4.31 & $.0 S$ & 260.10 & 4.42 & .09 \\
\hline 266.09 & 4.18 & .09 & 261.09 & 4.43 & .09 \\
\hline
\end{tabular}


TABLE V (Contd.)

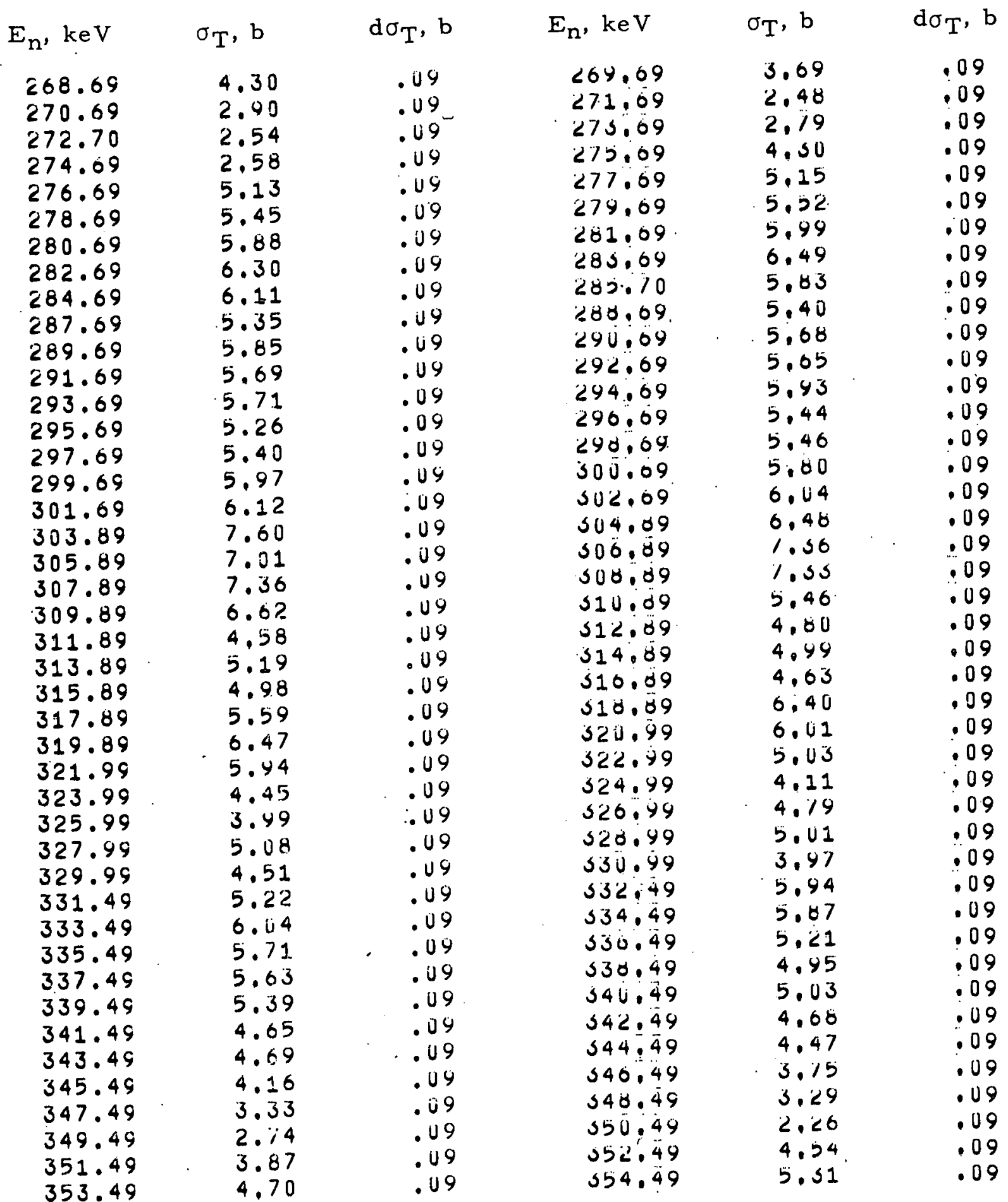


TABLE V (Contd.)

\begin{tabular}{|c|c|c|c|c|c|}
\hline$E_{n}$, keV & $\sigma_{\mathrm{T}}, \mathrm{b}$ & $\mathrm{d} \sigma_{\mathrm{T}}, \mathrm{b}$ & $E_{n}, \mathrm{keV}$ & $\sigma_{\mathrm{T}}, \mathrm{b}$ & $d \sigma_{T}, k$ \\
\hline $\begin{array}{l}355.49 \\
357.49 \\
359.49 \\
361.59 \\
363.59 \\
365.59 \\
367.59 \\
369.59 \\
371.59 \\
373.59 \\
375.60 \\
377.59 \\
379.59 \\
381.60 \\
383.59 \\
385.59 \\
387.59 \\
387.89 \\
389.89 \\
392.89 \\
394.89 \\
396.89 \\
398.89 \\
400.89 \\
402.89 \\
404.89 \\
406.89 \\
408.89 \\
410.89 \\
413.09 \\
415.09 \\
417.09 \\
419.09 \\
421.09 \\
423.09 \\
425.09 \\
427.09 \\
429.09 \\
431.09 \\
433.09 \\
435.09 \\
437.09 \\
439.09\end{array}$ & $\begin{array}{l}5.67 \\
6.58 \\
5.24 \\
4.42 \\
4.21 \\
4.29 \\
4.76 \\
4.99 \\
5.00 \\
4.90 \\
4.42 \\
3.68 \\
3.06 \\
3.05 \\
2.88 \\
3.10 \\
2.09 \\
1.96 \\
2.36 \\
2.53 \\
3.76 \\
4.71 \\
4.16 \\
4.07 \\
4.01 \\
4.13 \\
4.70 \\
4.44 \\
4.22 \\
4.23 \\
2.56 \\
2.41 \\
2.09 \\
2.27 \\
2.20 \\
2.23 \\
2.84 \\
3.43 \\
3.33 \\
3.79 \\
4.58 \\
5.09 \\
4.59\end{array}$ & $\begin{array}{l}.09 \\
.09 \\
.09 \\
.09 \\
.09 \\
.09 \\
.09 \\
.09 \\
.09 \\
.09 \\
.09 \\
.09 \\
.09 \\
.09 \\
.09 \\
.09 \\
.09 \\
.09 \\
.09 \\
.09 \\
.09 \\
.09 \\
.09 \\
.09 \\
.09 \\
.09 \\
.09 \\
.09 \\
.09 \\
.09 \\
.09 \\
.09 \\
.09 \\
.09 \\
.09 \\
.09 \\
.09 \\
.09 \\
.09 \\
.09 \\
.09 \\
.09 \\
.09\end{array}$ & 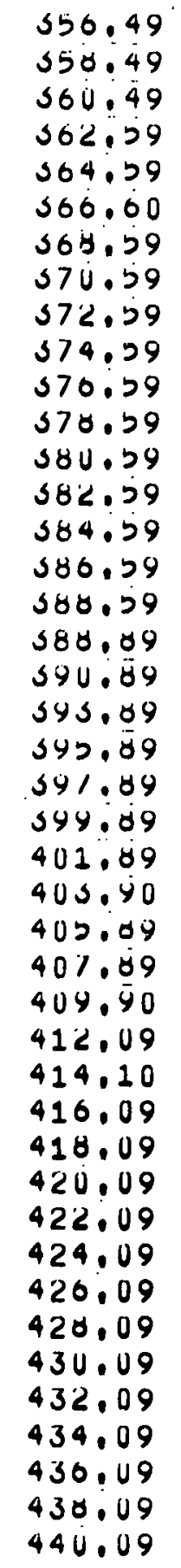 & 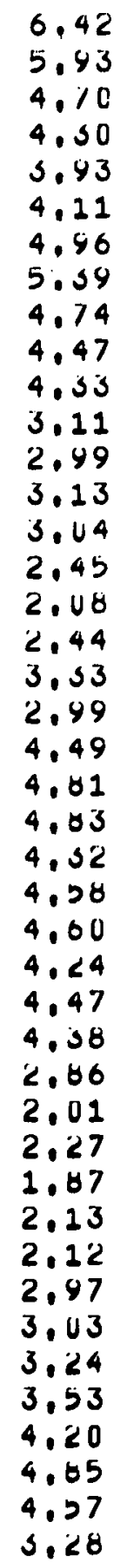 & $\begin{array}{l}.09 \\
.09 \\
.09 \\
.09 \\
.09 \\
.09 \\
.09 \\
.09 \\
.09 \\
.09 \\
.09 \\
.09 \\
.09 \\
.09 \\
.09 \\
.09 \\
.09 \\
.09 \\
.09 \\
.09 \\
.09 \\
.09 \\
.09 \\
.09 \\
.09 \\
.09 \\
.09 \\
.09 \\
.09 \\
.09 \\
.09 \\
.09 \\
.09 \\
.09 \\
.09 \\
.09 \\
.09 \\
.09 \\
.09 \\
.09 \\
.09 \\
.09 \\
.09\end{array}$ \\
\hline
\end{tabular}


TABLE V (Contd.)

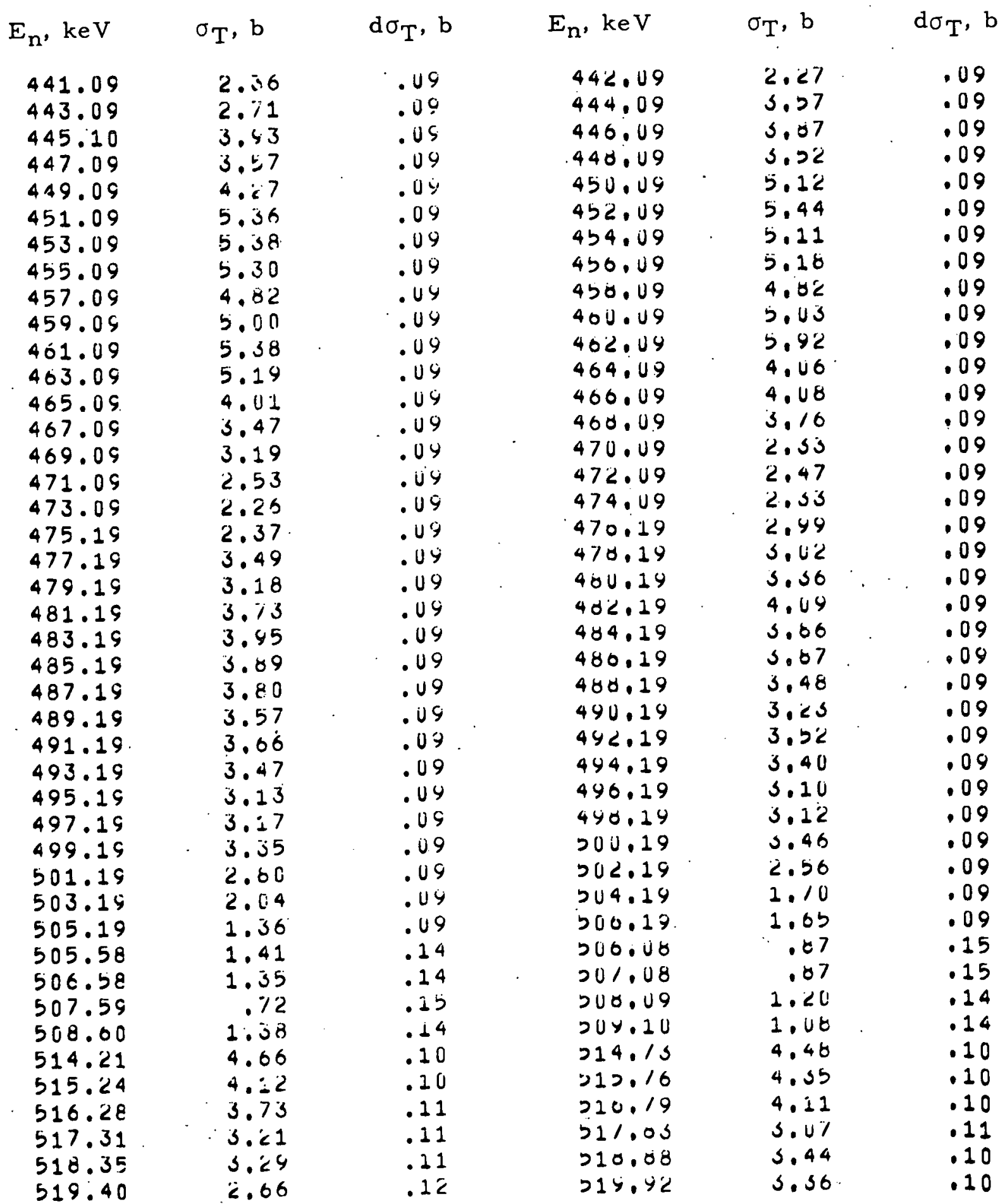


TABLE V (Contd.)

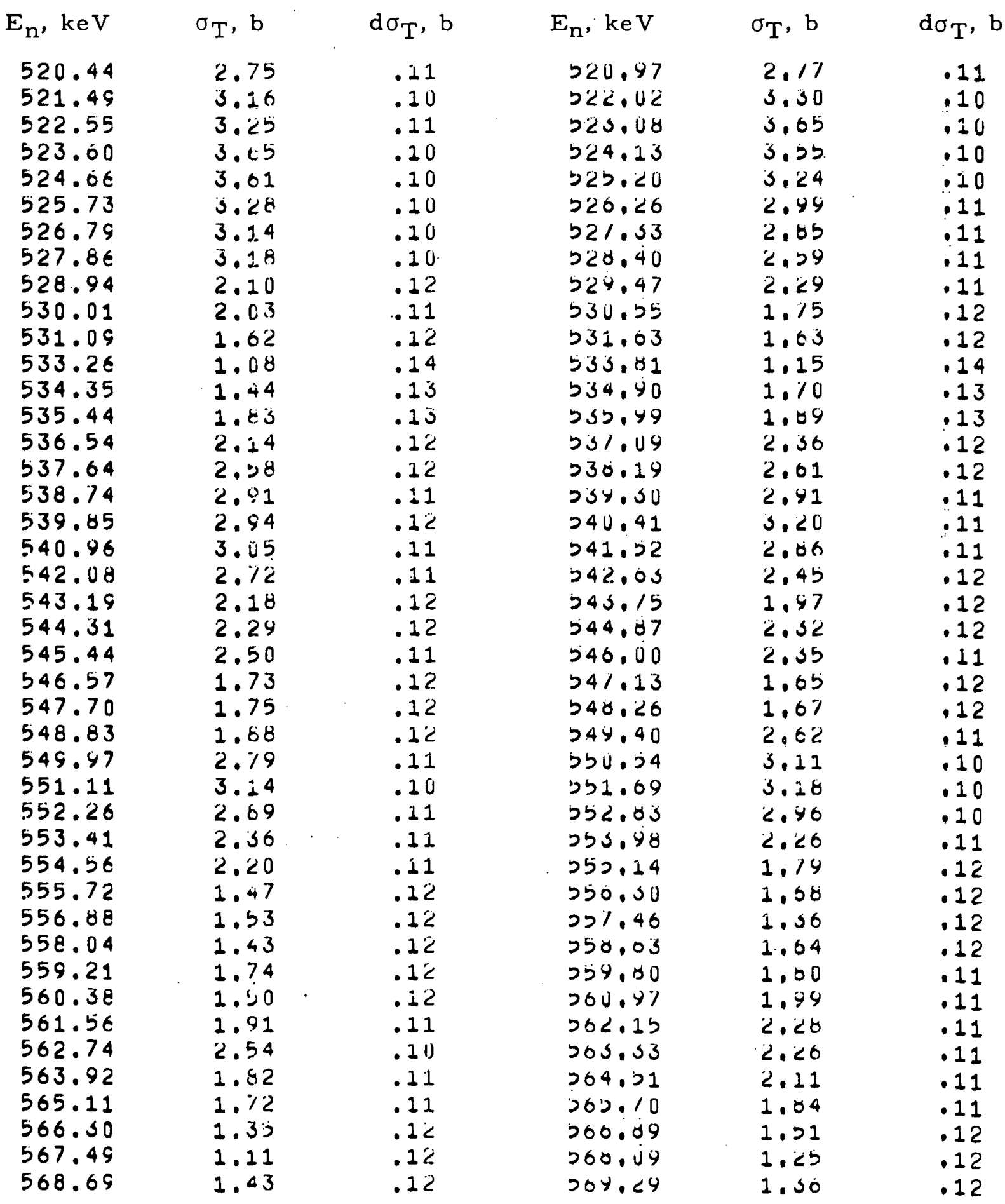


TABLE V (Contd.)

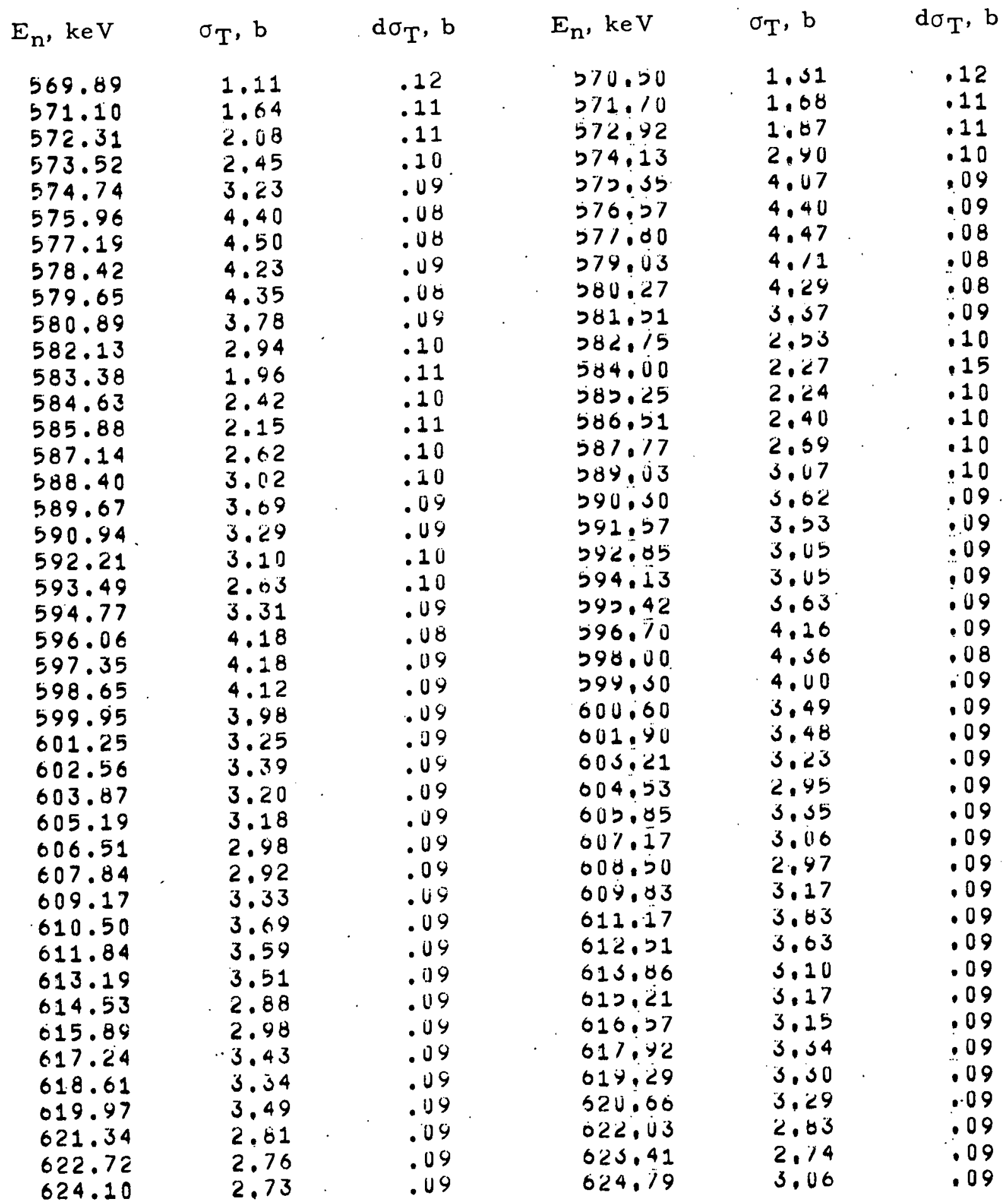


TABLE V (Contd.)

\begin{tabular}{|c|c|c|c|c|c|}
\hline$E_{n}, \mathrm{keV}$ & $\sigma_{\mathrm{T}}, \mathrm{b}$ & $\mathrm{d} \sigma_{\mathrm{T}}, \mathrm{b}$ & $E_{n}$, keV & $\sigma_{\mathrm{T}}, \mathrm{b}$ & $d \sigma_{T}, 1$ \\
\hline 025.49 & $3 .<7$ & .09 & 026.18 & 3.16 & .09 \\
\hline 626.88 & 2.48 & .10 & $62 \% .57$ & 2.10 & .10 \\
\hline 628.27 & 2.12 & .10 & 628,97 & 2.20 & .10 \\
\hline 629.67 & 1.81 & .10 & 63 is.j7 & 1.97 & .10 \\
\hline 031.07 & 1.46 & .15 & 051.18 & 1.32 & .11 \\
\hline 032.48 & 1.59 & .10 & 035.19 & 1.47 & .10 \\
\hline 633.89 & 2.57 & .09 & 034.00 & 3.45 & .09 \\
\hline 035.31 & 3.03 & .08 & 030.42 & 3.49 & .09 \\
\hline 036.74 & 3.77 & .09 & $63 \% .45$ & 4.107 & .08 \\
\hline 638.16 & 4.15 & .08 & 630.88 & 4,21 & .08 \\
\hline 639.60 & 4.49 & .08 & $64 \mathrm{U} .32$ & 4.16 & .08 \\
\hline 641.04 & 3.98 & .08 & 041.76 & 3.40 & .08 \\
\hline 042.48 & 3.96 & .08 & 045.20 & $3.4 U$ & .09 \\
\hline 043.93 & 3.00 & .09 & 044.05 & 2.81 & .09 \\
\hline 645.38 & 2.42 & .10 & 040.11 & 2.13 & .10 \\
\hline 646.84 & 2.34 & .15 & 047.07 & 2.29 & .15 \\
\hline 048.30 & 2.35 & .11 & 043.03 & 2.29 & .11 \\
\hline 649.77 & 2.76 & .11 & cbU: 50 & 3.43 & .10 \\
\hline 651.24 & 3.55 & .10 & 651.98 & 3.10 & .10 \\
\hline 652.72 & 3.83 & .10 & 053.46 & 4.10 & .09 \\
\hline 654.20 & 4.15 & .09 & 654.94 & 4.80 & .09 \\
\hline 655.69 & 4.97 & .09 & 050.43 & 4.59 & .09 \\
\hline 657.18 & 3.66 & .10 & 651.93 & 3.16 & .10 \\
\hline 658.68 & 3.20 & .10 & 659.43 & 3.40 & .10 \\
\hline 660.18 & 3.77 & .10 & 060.94 & 3.92 & .09 \\
\hline 661.69 & 4.31 &.$\cup \varphi$ & 062.45 & 4.62 & .09 \\
\hline 663.21 & 4.83 & .09 & 06.5 .96 & 4.54 & .09 \\
\hline 664.72 & 4.49 & .09 & 005.49 & 4.16 & .09 \\
\hline 666.25 & 4.01 & .49 & $06 \% .01$ & 3.48 & .10 \\
\hline 667.76 & 2.77 & .10 & 068.55 & 2.44 & .11 \\
\hline 669.31 & 2.42 & .11 & 070.08 & 2.04 & .11 \\
\hline 670.86 & 2.12 & .11 & 671.03 & 2.37 & .11 \\
\hline 672.40 & 2.29 & .11 & 075.18 & 2.66 & .11 \\
\hline 673.95 & 3.20 & .10 & 674.73 & 3.00 & .10 \\
\hline 075.51 & 3.03 & .10 & $676 .<9$ & $2,<7$ & .11 \\
\hline 677.07 & 2.69 & .20 & 611.86 & 2.00 & .11 \\
\hline 678.04 & 2.15 & .21 & 079.43 & 2. 11. & .11 \\
\hline 680.21 & 1.74 & .12 & $001 . \overline{0} 0$ & 1.06 & .12 \\
\hline 681.79 & 2.41 & .11 & 082.58 & 2.65 & .10 \\
\hline 683.38 & 3.15 & .10 & 686.26 & 4.16 & .09 \\
\hline 687.36 & 4.17 & .09 & 000.16 & $4 \cdot<8$ & .09 \\
\hline 688.97 & 4.57 & .08 & 084.17 & 4.50 & .09 \\
\hline 690.58 & 4,80 & .08 & 641.58 & 4.47 & .09 \\
\hline
\end{tabular}


TABLE V (Contd.)

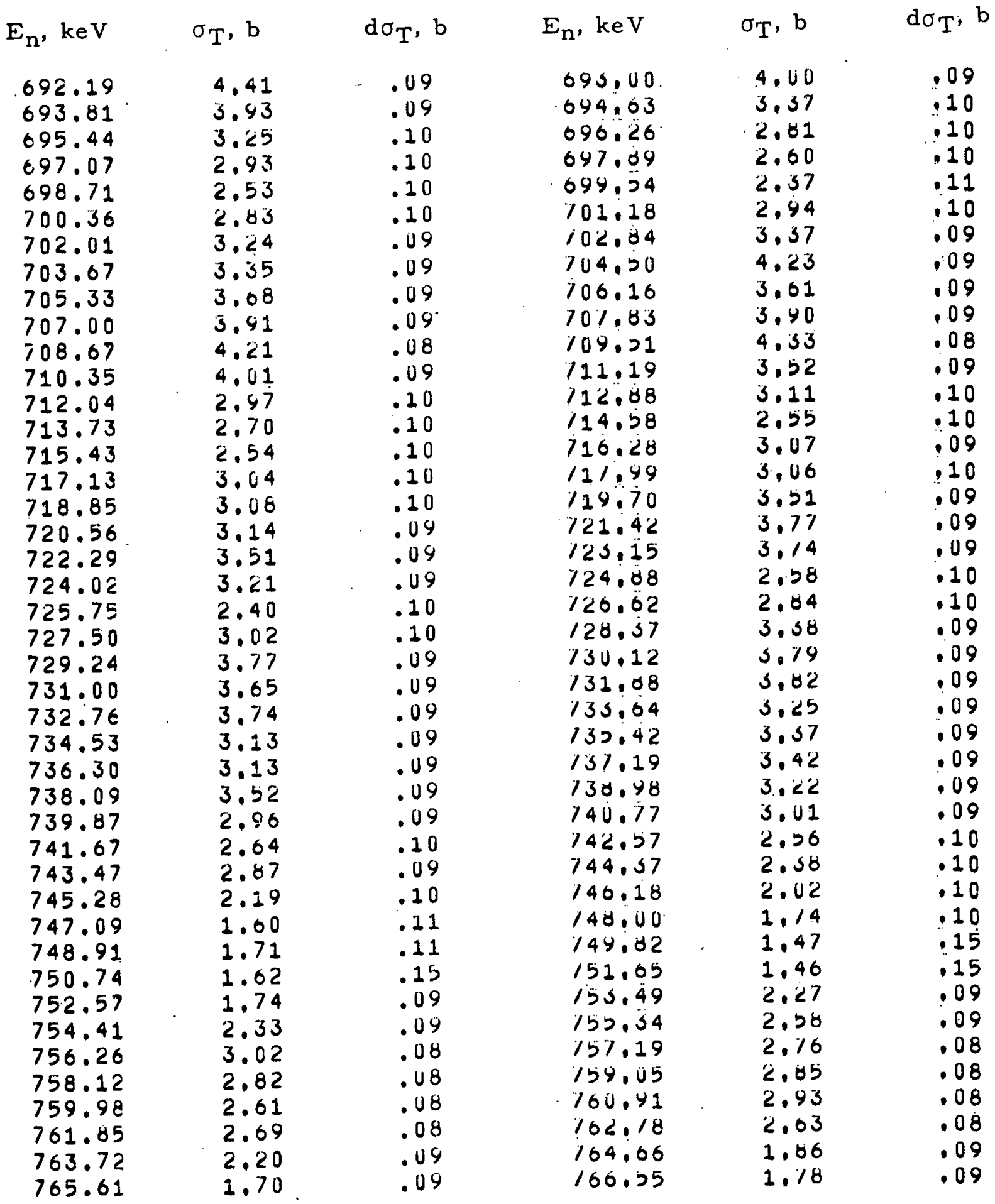


TABLE V (Contd.)

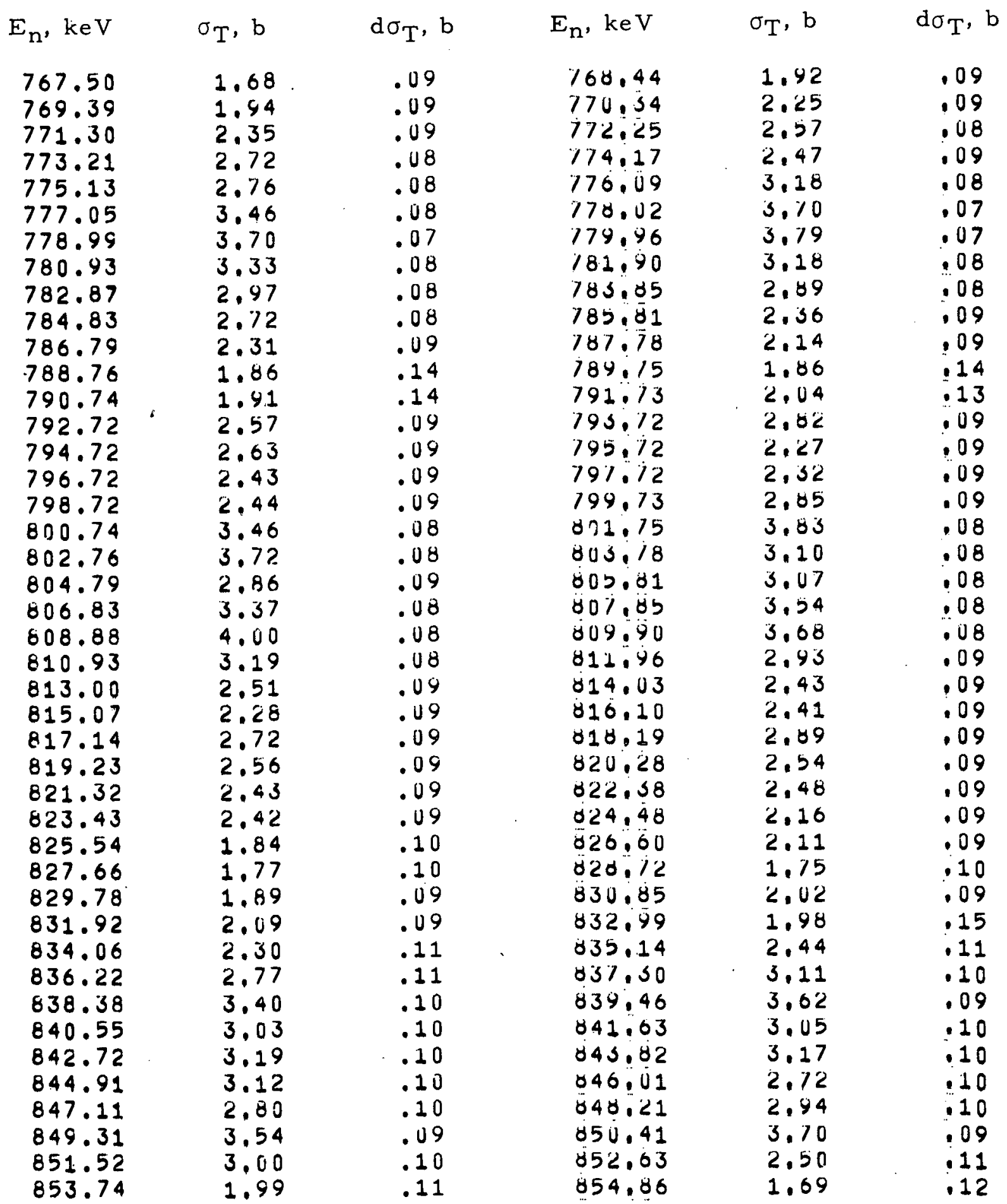


TABLE V (Contd.)

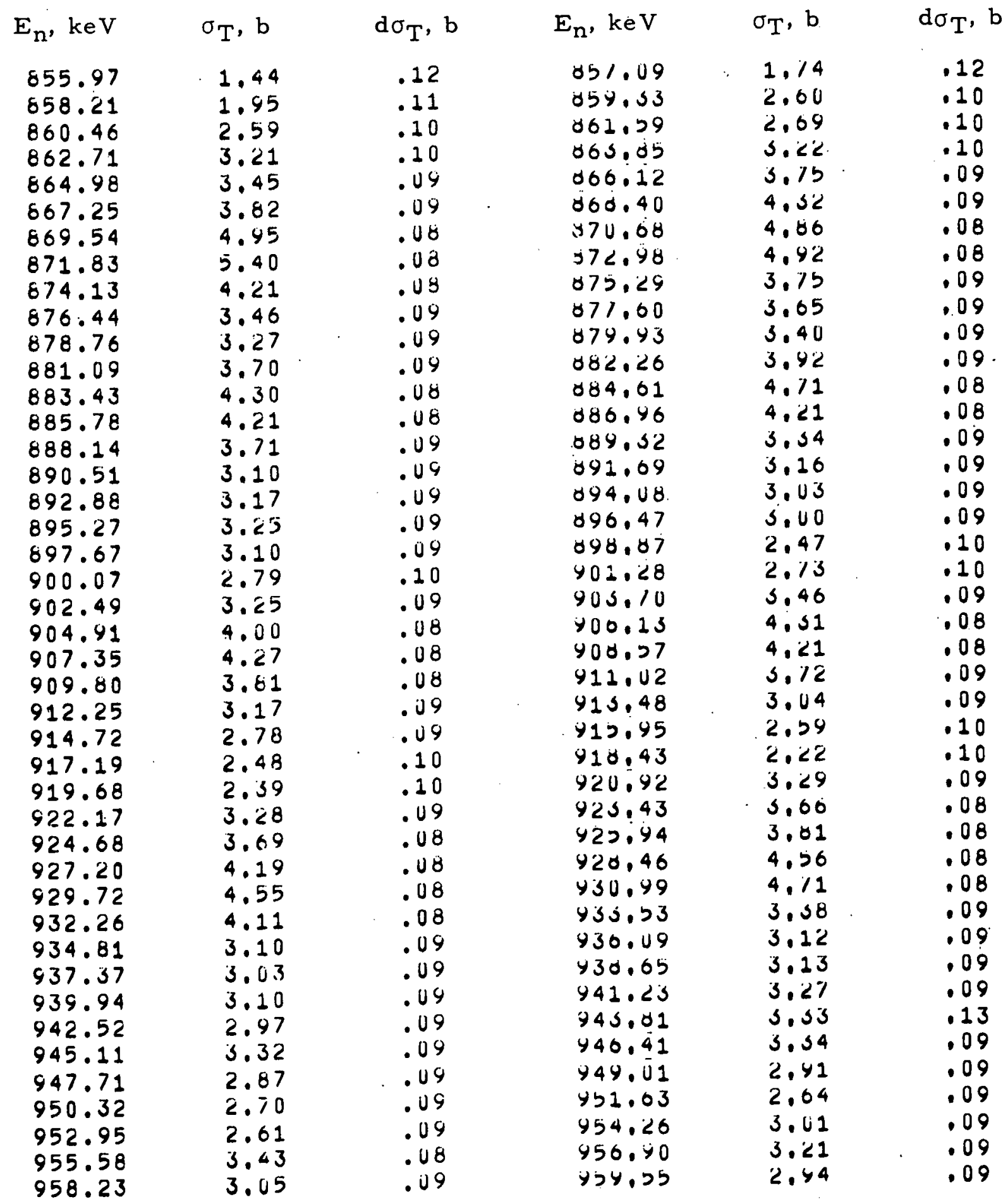


TABLE V (Contd.)

\begin{tabular}{|c|c|c|c|c|c|}
\hline$E_{n}, k e V$ & $\sigma_{\mathrm{T}}, \mathrm{b}$ & $\mathrm{d} \sigma_{\mathrm{T}}, \mathrm{b}$ & $E_{n}, \mathrm{keV}$ & $\sigma_{\mathrm{T}}, \mathrm{b}$ & $\mathrm{d} \sigma_{\mathrm{T}}, \mathrm{b}$ \\
\hline $\begin{array}{l}960.88 \\
963.55 \\
966.23 \\
970.27 \\
972.97 \\
975.69 \\
978.42 \\
981.16 \\
983.92 \\
986.68 \\
989.46 \\
992.25 \\
995.05 \\
997.86 \\
1000.69 \\
1003.52 \\
1006.37 \\
1009.23 \\
1012.11 \\
1014.99 \\
1017.89 \\
1019.34 \\
1022.26 \\
1025.19 \\
1028.13 \\
1031.09 \\
1034.06 \\
1037.04 \\
1041.53 \\
1044.55 \\
1047.58 \\
1050.62 \\
1053.67 \\
1056.74 \\
1059.82 \\
1062.91 \\
1066.02 \\
1069.14 \\
1072.28 \\
1075.43 \\
1078.59 \\
1081.77 \\
1084.96\end{array}$ & $\begin{array}{l}2.59 \\
2.68 \\
3.15 \\
4.26 \\
4.12 \\
2.75 \\
3.44 \\
3.75 \\
3.18 \\
2.77 \\
2.75 \\
2.95 \\
3.05 \\
2.92 \\
2.65 \\
2.83 \\
3.17 \\
2.84 \\
3.19 \\
3.77 \\
3.79 \\
3.25 \\
2.67 \\
2.54 \\
2.38 \\
2.33 \\
2.34 \\
2.38 \\
2.52 \\
3.09 \\
2.65 \\
2.15 \\
2.15 \\
2.79 \\
2.93 \\
2.70 \\
2.71 \\
3.20 \\
3.18 \\
3.16 \\
3.32 \\
3.25 \\
3.44\end{array}$ & $\begin{array}{l}.09 \\
.09 \\
.09 \\
.01 \\
.08 \\
.09 \\
.08 \\
.08 \\
.09 \\
.09 \\
.09 \\
.09 \\
.09 \\
.09 \\
.10 \\
.10 \\
.10 \\
.10 \\
.09 \\
.09 \\
.09 \\
.09 \\
.10 \\
.10 \\
.100 \\
.10 \\
.10 \\
.10 \\
.10 \\
.09 \\
.09 \\
.10 \\
.10 \\
.14 \\
.13 \\
.09 \\
.09 \\
.09 \\
.09 \\
.09 \\
.09 \\
.09 \\
.08\end{array}$ & 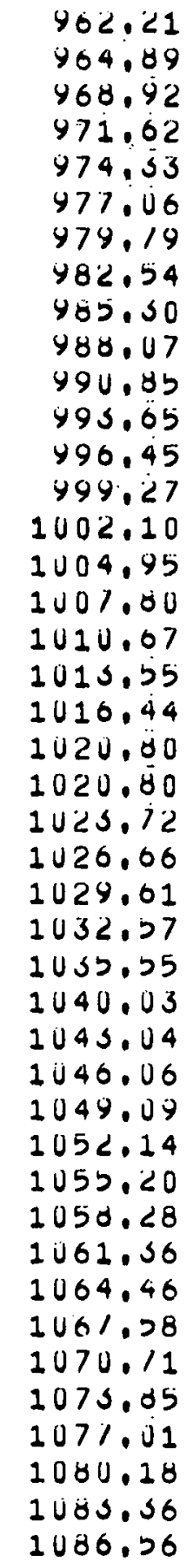 & $\begin{array}{l}2.42 \\
2.62 \\
3.90 \\
4.02 \\
3.76 \\
3.26 \\
3.79 \\
3.49 \\
2.83 \\
2.17 \\
2.86 \\
2.95 \\
2.65 \\
3.27 \\
3.04 \\
3.24 \\
3.33 \\
2.04 \\
3.47 \\
4.04 \\
3.22 \\
3.22 \\
2.68 \\
2.31 \\
2.03 \\
2.44 \\
2.45 \\
2.31 \\
2.43 \\
2.68 \\
2.38 \\
2.58 \\
2.47 \\
2.85 \\
3.17 \\
2.92 \\
2.75 \\
3.08 \\
5.04 \\
3.28 \\
3.20 \\
3.31 \\
3.30\end{array}$ & 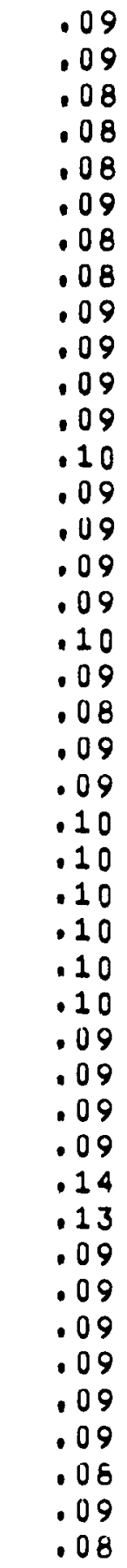 \\
\hline
\end{tabular}


TABLE V (Contd.)

\begin{tabular}{|c|c|c|c|c|c|}
\hline$E_{n}, k e V$ & $\sigma_{\mathrm{T}}, \mathrm{b}$ & $\mathrm{d} \sigma_{\mathrm{T}}, \mathrm{b}$ & $E_{n}, \mathrm{keV}$ & $\sigma_{T}, b$ & $d \sigma_{T}, b$ \\
\hline $\begin{array}{l}1088.17 \\
1091.39 \\
1094.62 \\
1099.50 \\
1102.77 \\
1106.06 \\
1109.36 \\
1112.68 \\
1116.01 \\
1119.35 \\
1122.71 \\
1126.09 \\
1129.48 \\
1132.89 \\
1136.31 \\
1139.75 \\
1143.21 \\
1146.68 \\
1150.16 \\
1153.67 \\
1157.18 \\
1160.72 \\
1164.27 \\
1167.84 \\
1171.42 \\
1175.02 \\
1178.64 \\
1182.27 \\
1185.92 \\
1189.59 \\
1193.28 \\
1196.98 \\
1200.70 \\
1204.44 \\
1208.19 \\
1211.97 \\
1215.76 \\
1219.57 \\
1223.39 \\
1227.24 \\
1231.10 \\
1234.98 \\
1238.88\end{array}$ & $\begin{array}{l}3.46 \\
3.27 \\
3.31 \\
3.12 \\
4.15 \\
4.16 \\
4.15 \\
3.49 \\
3.29 \\
3.96 \\
4.02 \\
3.55 \\
3.48 \\
3.40 \\
3.26 \\
4.12 \\
4.10 \\
2.64 \\
2.65 \\
2.87 \\
3.53 \\
3.35 \\
2.79 \\
3.05 \\
3.61 \\
3.49 \\
4.07 \\
3.82 \\
3.16 \\
2.96 \\
3.31 \\
3.54 \\
3.06 \\
3.30 \\
2.98 \\
2.67 \\
2.47 \\
3.23 \\
3.30 \\
3.44 \\
3.51 \\
2.68 \\
3.54\end{array}$ & $\begin{array}{l}.08 \\
.09 \\
.09 \\
.14 \\
.09 \\
.09 \\
.09 \\
.09 \\
.09 \\
.08 \\
.08 \\
.08 \\
.08 \\
.08 \\
.08 \\
.07 \\
.07 \\
.08 \\
.08 \\
.08 \\
.07 \\
.07 \\
.08 \\
.08 \\
.07 \\
.07 \\
.07 \\
.07 \\
.07 \\
.07 \\
.07 \\
.07 \\
.07 \\
.07 \\
.07 \\
.08 \\
.08 \\
.07 \\
.07 \\
.07 \\
.07 \\
.12 \\
.08 \\
.07 \\
.07 \\
.07\end{array}$ & 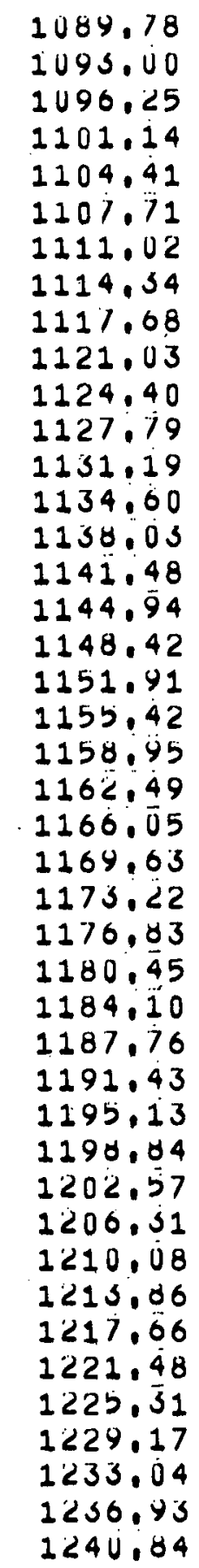 & $\begin{array}{l}3.50 \\
3.47 \\
3.48 \\
3.84 \\
4.65 \\
4.31 \\
3.75 \\
3.44 \\
3.52 \\
4.09 \\
3.61 \\
3.44 \\
3.38 \\
2.94 \\
3.54 \\
4.51 \\
3.32 \\
2.59 \\
2.82 \\
3.34 \\
3.79 \\
2.97 \\
2.93 \\
3.72 \\
3.89 \\
3.87 \\
4.03 \\
3.63 \\
3.03 \\
3.15 \\
3.43 \\
3.60 \\
3.13 \\
3.46 \\
2.89 \\
2.64 \\
2.75 \\
3.14 \\
3.53 \\
3.75 \\
2.88 \\
3.25 \\
3.60\end{array}$ & 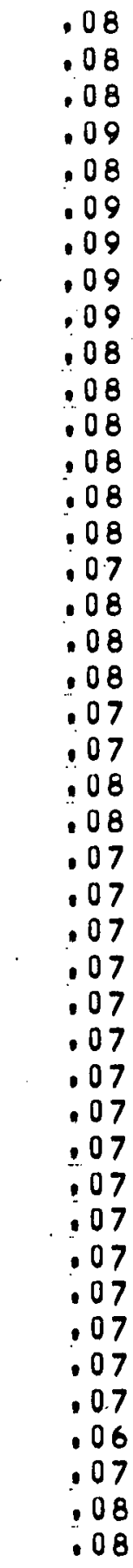 \\
\hline
\end{tabular}


TABLE V (Contd.)

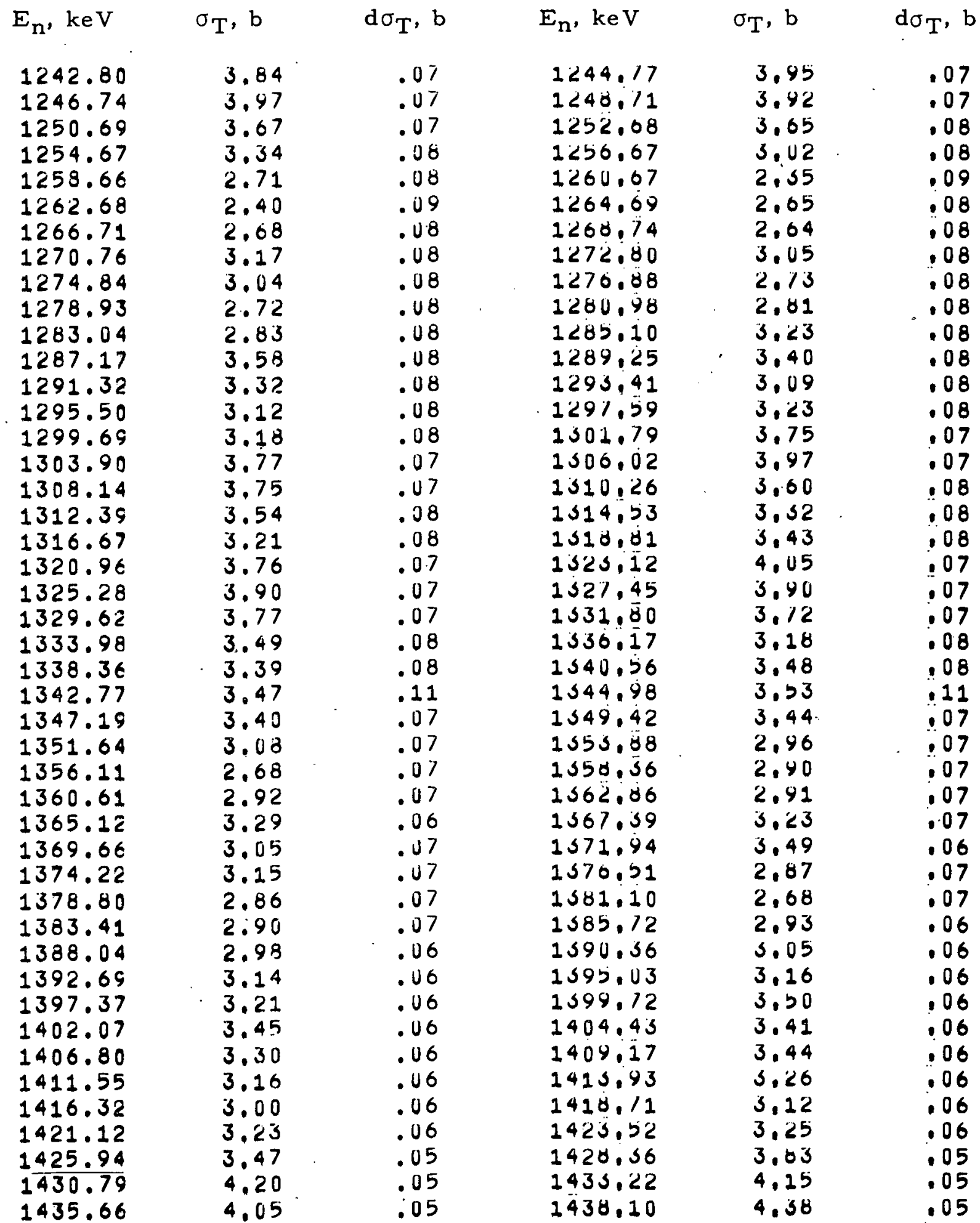


TABLE VI. Differential Elastic Scattering Cross Sections of Vanadium, Expressed in Format of Eq. 1

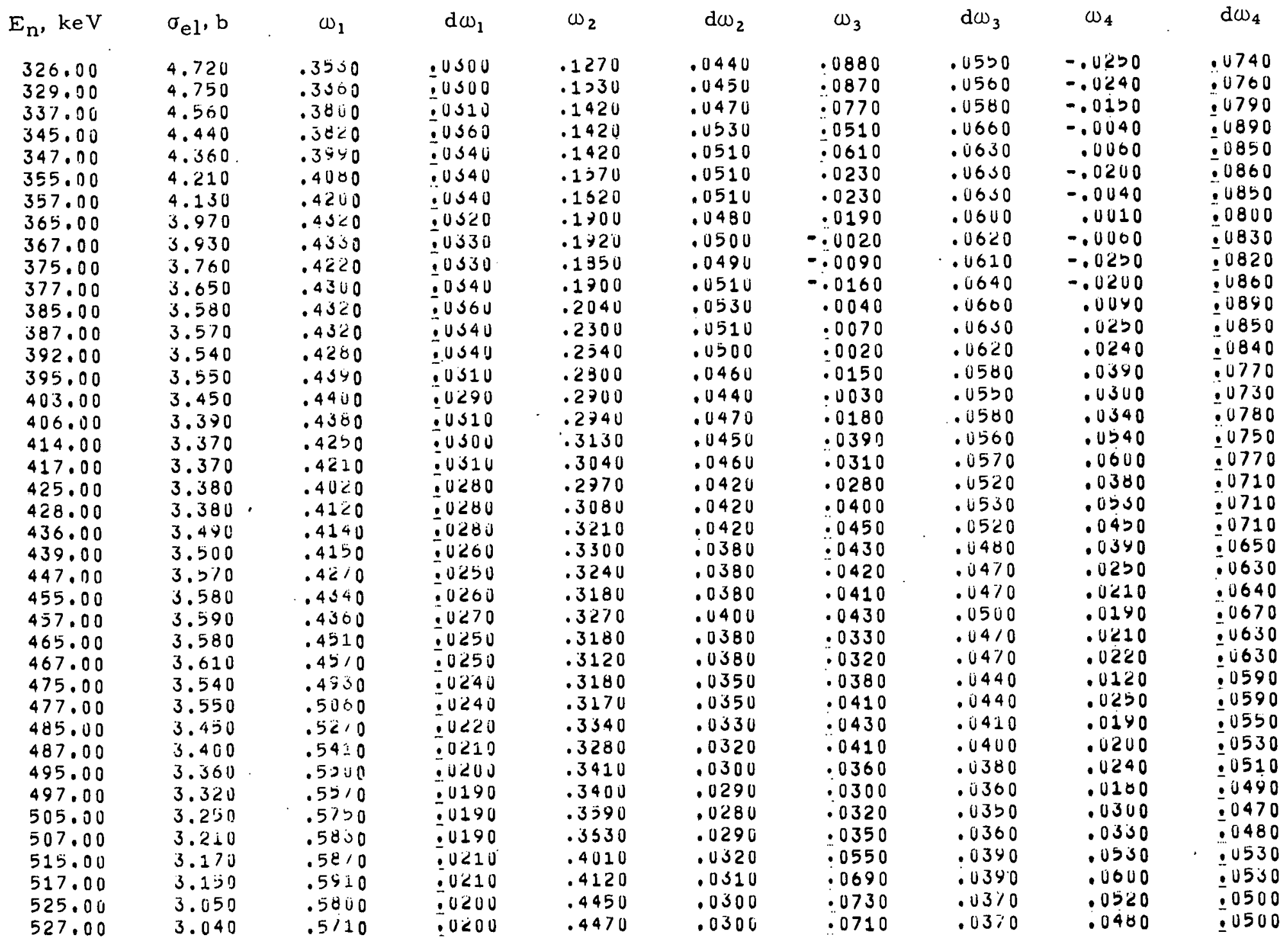


TABLE VI (Contd.)

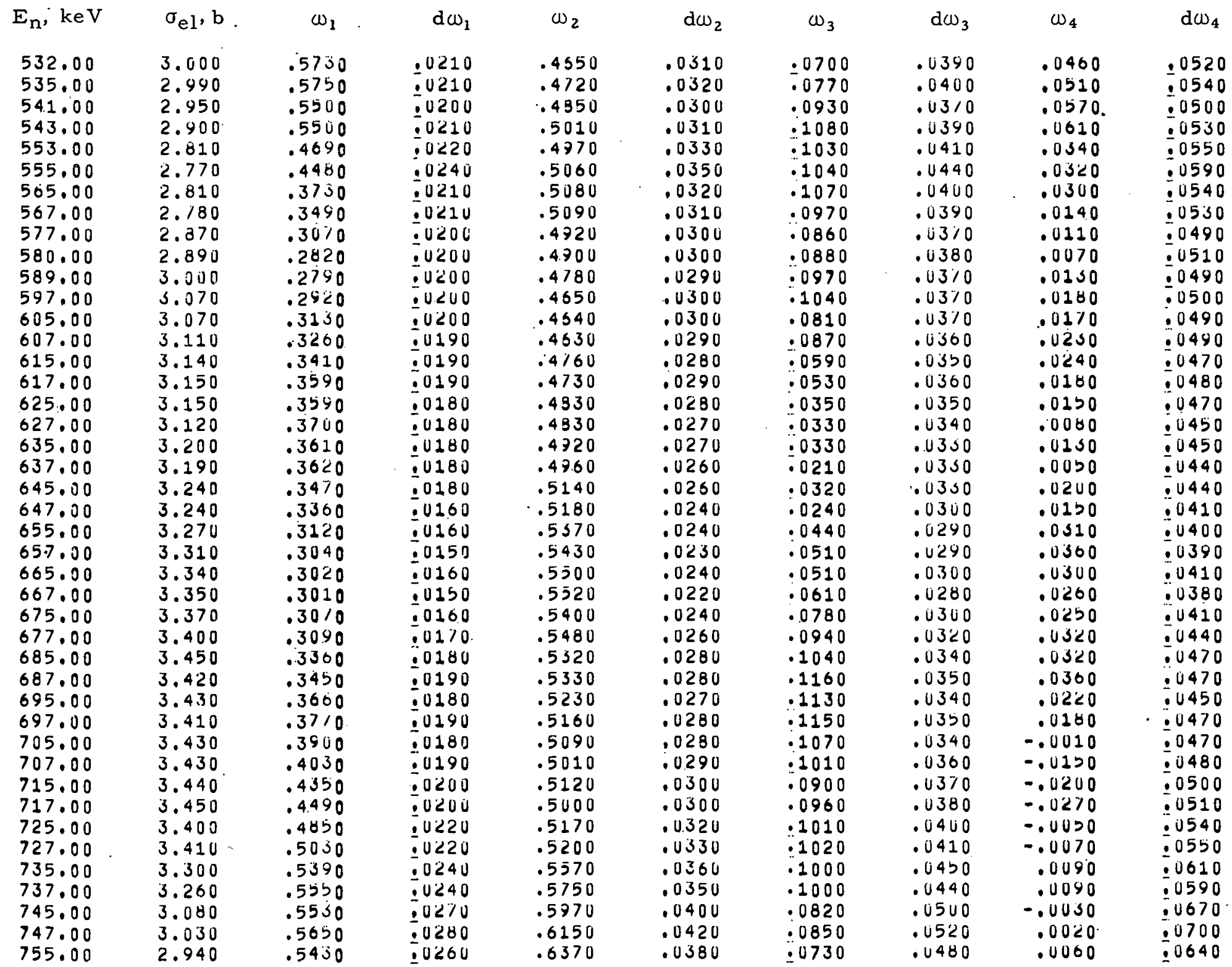




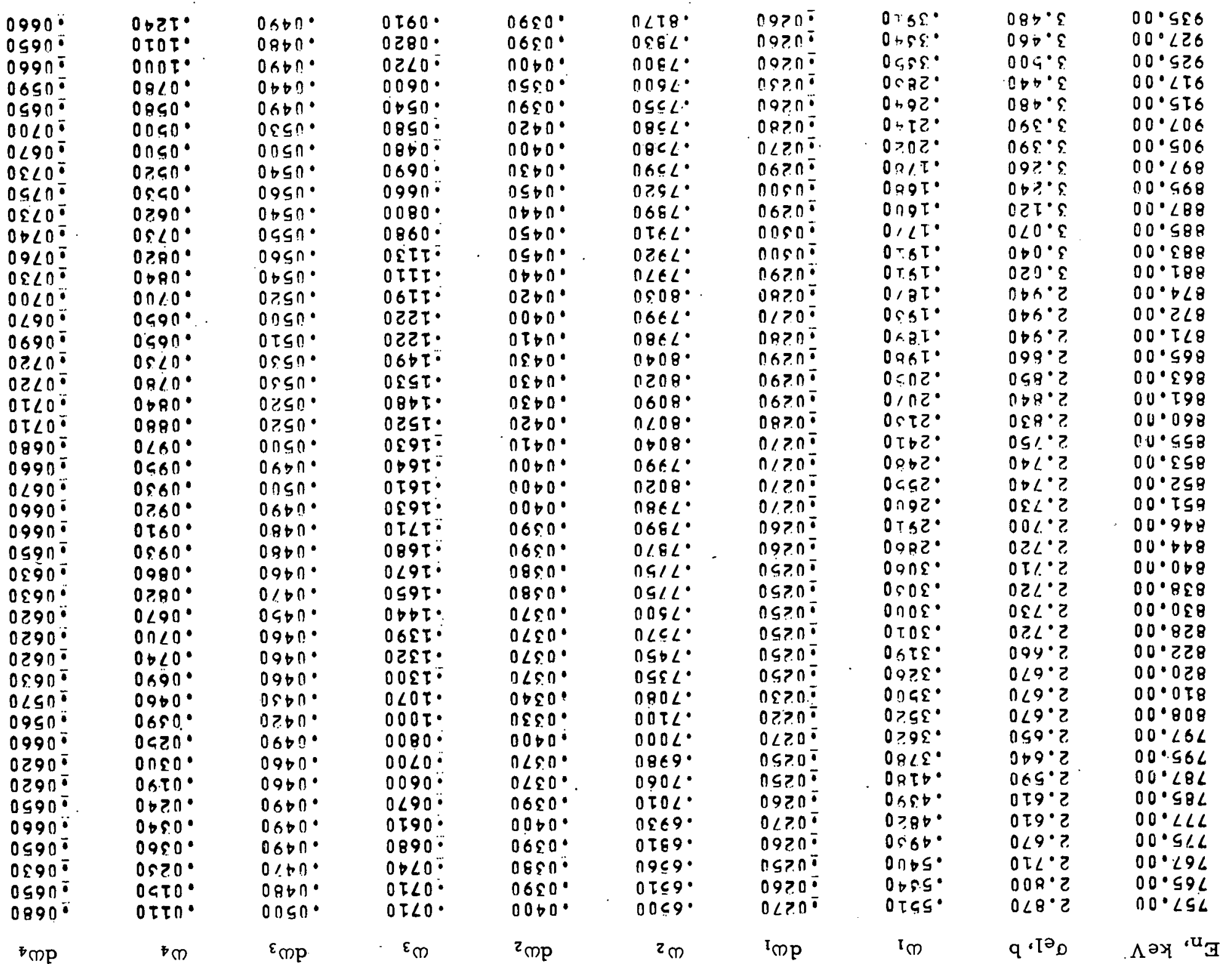

('płuoว) $\Lambda$ I JTgVI 
TABLE IV (Contd.)

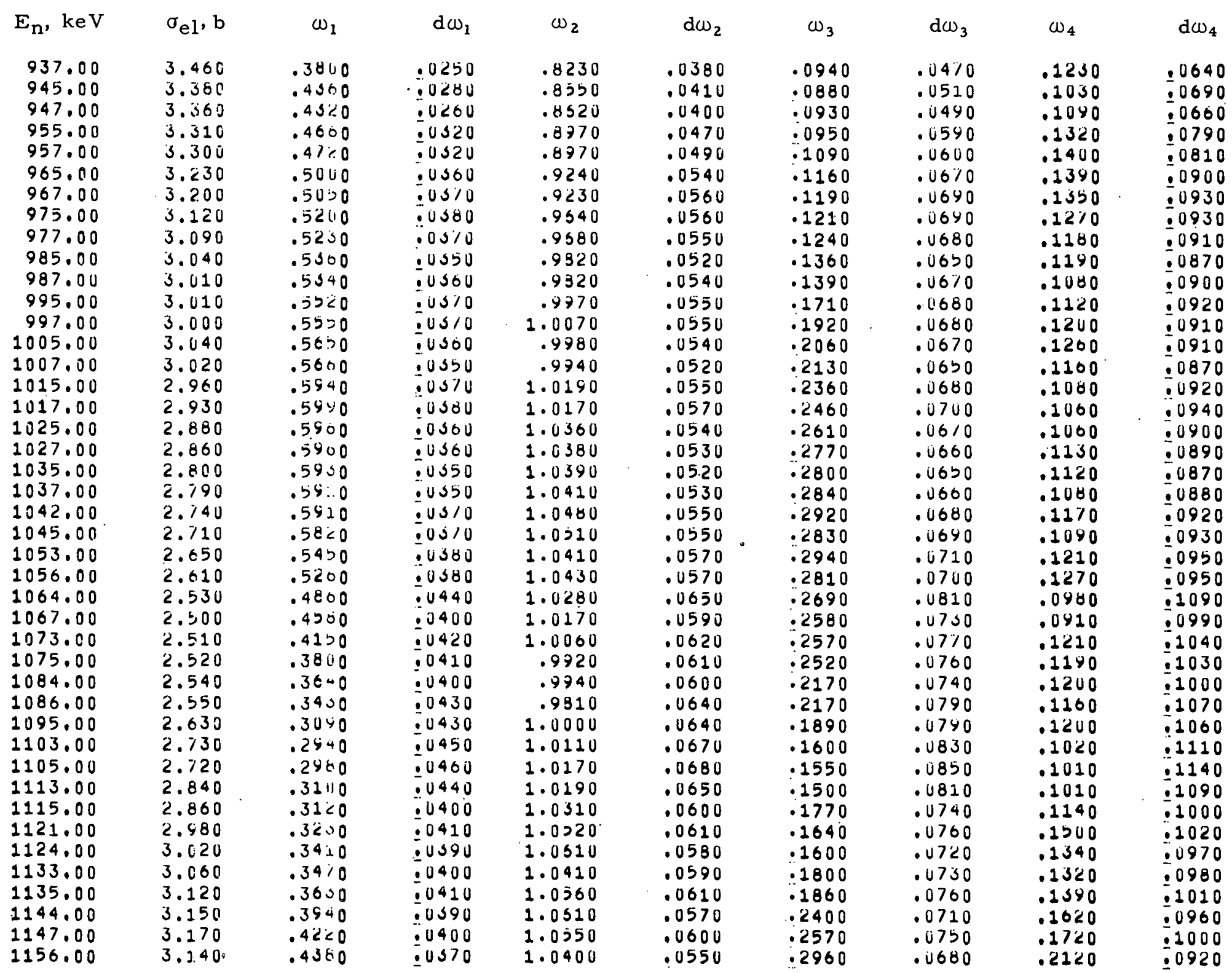


TABLE VI (Contd.)

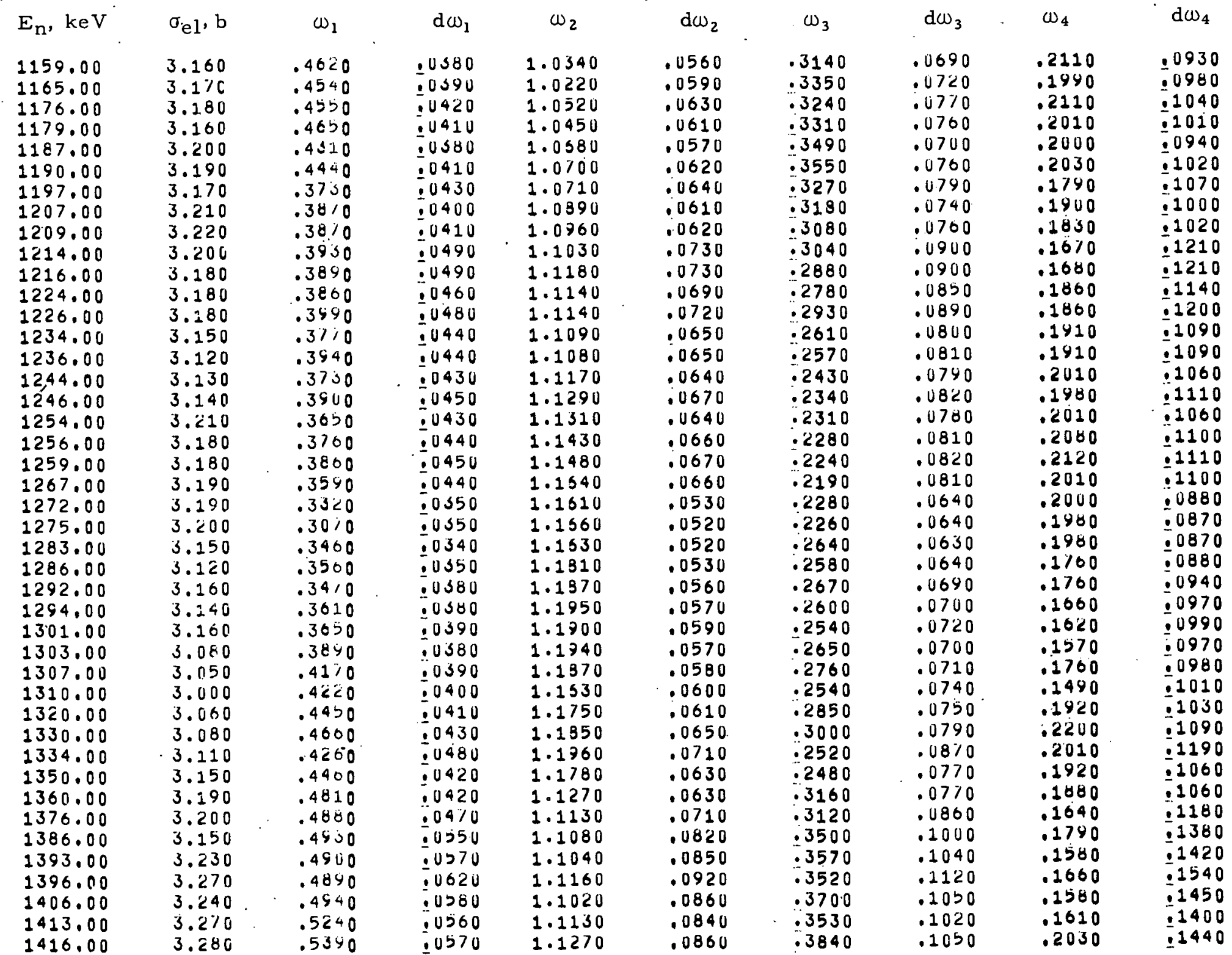


TABLE VI (Contd.)

\begin{tabular}{|c|c|c|c|c|c|c|c|c|c|}
\hline$E_{n}$, keV & $\sigma_{e l}, b$ & $\omega_{1}$ & $\mathrm{~d} \omega_{1}$ & $\omega_{2}$ & $\mathrm{~d} \omega_{2}$ & $\omega_{3}$ & $\mathrm{~d} \omega_{3}$ & $\omega_{4}$ & $\mathrm{~d} \omega_{4}$ \\
\hline $\begin{array}{l}1426.00 \\
1433.00 \\
1436.00 \\
1443.00 \\
1447.00 \\
1450.00 \\
1460.00 \\
1464.00 \\
1467.00\end{array}$ & $\begin{array}{l}3.260 \\
3.270 \\
3.270 \\
3.260 \\
3.220 \\
3.190 \\
3.360 \\
3.310 \\
3.340\end{array}$ & $\begin{array}{l}.5040 \\
.5290 \\
.5450 \\
.5030 \\
.5040 \\
.4840 \\
.4920 \\
.4370 \\
.3980\end{array}$ & $\begin{array}{l}.0510 \\
.0520 \\
.0500 \\
.0420 \\
.0580 \\
.0390 \\
.0410 \\
-0430 \\
.0370\end{array}$ & $\begin{array}{l}1.1310 \\
1.1330 \\
1.1460 \\
1.1170 \\
1.1230 \\
1.1440 \\
1.1460 \\
1.1590 \\
1.1390\end{array}$ & $\begin{array}{l}.0770 \\
.0780 \\
.0760 \\
.0630 \\
.0570 \\
.0590 \\
.0610 \\
.0650 \\
.0550\end{array}$ & $\begin{array}{r}.3650 \\
.4110 \\
.4310 \\
.3750 \\
.3620 \\
.3940 \\
.3590 \\
.3660 \\
.3460\end{array}$ & $\begin{array}{l}.0940 \\
.0950 \\
.0920 \\
.0760 \\
.0690 \\
.0720 \\
.0740 \\
.0790 \\
.0680\end{array}$ & $\begin{array}{l}.1920 \\
.2130 \\
.2290 \\
.1750 \\
.1940 \\
.2360 \\
.2600 \\
.2960 \\
.3080\end{array}$ & $\begin{array}{l}.1290 \\
.1300 \\
11260 \\
11050 \\
: 0950 \\
: 0990 \\
11020 \\
.1080 \\
.0930\end{array}$ \\
\hline
\end{tabular}


TABLE VII. Cross Sections for the Inelastic Excitations of the $320-\mathrm{keV}$ State in Vanadium by Neutron Scattering

\begin{tabular}{|c|c|c|c|c|c|}
\hline $\mathrm{E}_{\mathrm{n}}, \mathrm{MeV}$ & $\sigma_{\text {in }}, b$ & $\mathrm{~d} \sigma_{i n}, \mathrm{~b}$ & $\mathrm{E}_{\mathrm{n}}, \mathrm{MeV}$ & $\sigma_{\text {in }}, b$ & $\mathrm{~d} \sigma_{\mathrm{in}}, \mathrm{b}$ \\
\hline $\begin{array}{l}.650 \\
.074 \\
.686 \\
.695 \\
.716 \\
.727 \\
.734 \\
.740 \\
.753 \\
.756 \\
.767 \\
.774 \\
.785 \\
.794 \\
.804 \\
.813 \\
.827 \\
.834 \\
.844 \\
.854 \\
.865 \\
.873 \\
.876 \\
.886 \\
.896 \\
.906 \\
.916 \\
.926 \\
.936 \\
.944 \\
.963 \\
.976 \\
.986 \\
.989 \\
1.006 \\
1.010 \\
1.035 \\
1.043 \\
1.055 \\
1.076\end{array}$ & $\begin{array}{l}.330 \\
.389 \\
.406 \\
.475 \\
.383 \\
.388 \\
.356 \\
.371 \\
.333 \\
.343 \\
.351 \\
.463 \\
.480 \\
.494 \\
.512 \\
.509 \\
.513 \\
.513 \\
.487 \\
.495 \\
.474 \\
.440 \\
.432 \\
.385 \\
.408 \\
.402 \\
.337 \\
.384 \\
.452 \\
.469 \\
.405 \\
.372 \\
.392 \\
.415 \\
.400 \\
.336 \\
.419 \\
.405 \\
.432 \\
.428\end{array}$ & $\begin{array}{l}.054 \\
.056 \\
.051 \\
.049 \\
.039 \\
.032 \\
.027 \\
.040 \\
.023 \\
.029 \\
.028 \\
.035 \\
.037 \\
.038 \\
.059 \\
.045 \\
.039 \\
.038 \\
.035 \\
.036 \\
.031 \\
.049 \\
.037 \\
.034 \\
.035 \\
.035 \\
.029 \\
.033 \\
.039 \\
.049 \\
.035 \\
.032 \\
.034 \\
.062 \\
.034 \\
.050 \\
.031 \\
.035 \\
.046 \\
.037\end{array}$ & $\begin{array}{l}.0500 \\
.0775 \\
.0453 \\
.1050 \\
.1259 \\
.1359 \\
.1366 \\
.1400 \\
.1550 \\
.1659 \\
.7759 \\
.7766 \\
.7866 \\
.7966 \\
.0066 \\
.8149 \\
.0359 \\
.8366 \\
.0466 \\
.8566 \\
.0674 \\
.8749 \\
.0849 \\
.0949 \\
.9049 \\
.9149 \\
.9249 \\
.9349 \\
.9453 \\
.9066 \\
.9649 \\
.9849 \\
.4899 \\
1.0050 \\
1.0100 \\
1.0200 \\
1.0360 \\
1.0450 \\
1.0650 \\
1.0850\end{array}$ & $\begin{array}{l}.2031 \\
.3805 \\
.3932 \\
.4247 \\
.3755 \\
.3118 \\
.3140 \\
.4750 \\
.3345 \\
.3520 \\
.4201 \\
.4049 \\
.4504 \\
.5551 \\
.4857 \\
.6041 \\
.4900 \\
.5242 \\
.4904 \\
.5162 \\
.4399 \\
.4443 \\
.3943 \\
.3918 \\
.3907 \\
.3496 \\
.3600 \\
.4562 \\
.4687 \\
.4317 \\
.4185 \\
.3427 \\
.4016 \\
.3953 \\
.3451 \\
.4435 \\
.4097 \\
.3420 \\
.4468\end{array}$ & $\begin{array}{l}.0391 \\
.0061 \\
.0572 \\
.0539 \\
.0274 \\
.0275 \\
.0352 \\
.0110 \\
.0251 \\
.0248 \\
.0302 \\
.0412 \\
.0403 \\
.0481 \\
.0434 \\
.0040 \\
.0332 \\
.0455 \\
.0403 \\
.0452 \\
.0324 \\
.0333 \\
.0302 \\
.0300 \\
.0300 \\
.0262 \\
.0276 \\
.0328 \\
.0406 \\
.0375 \\
.0444 \\
.0287 \\
.0426 \\
.0296 \\
.0423 \\
.0385 \\
.0356 \\
.0405 \\
.0474 \\
.0317\end{array}$ \\
\hline
\end{tabular}


TABLE VII (Contd.)

\begin{tabular}{|c|c|c|c|c|c|}
\hline$E_{n}, M e V$ & $\sigma_{\text {in }}, b$ & $\mathrm{~d} \sigma_{\mathrm{in}}, \mathrm{b}$ & $E_{n}, M e V$ & $\sigma_{\text {in }}, \mathrm{b}$ & $\mathrm{d} \sigma_{\text {in }}, \mathrm{b}$ \\
\hline 1.086 & .416 & .036 & 1.0950 & .4223 & $.0<86$ \\
\hline 1.097 & .433 & : & 1.1.10 10 & .44 .58 & .0301 \\
\hline 1.104 & .445 & .003 & 1.1060 & .4617 & .0400 \\
\hline 1.115 & .465 & .036 & 1.1100 & .5064 & .0438 \\
\hline 1.124 & .492 & .036 & 1.1200 & .4850 & .0420 \\
\hline 1.133 & .510 & .044 & 1.1つつ & .5220 & .0556 \\
\hline 1.145 & .528 & $.0=9$ & 1.1200 & .4822 & .0421 \\
\hline 1.163 & .420 & .036 & 1.1650 & .4367 & .0469 \\
\hline 1.176 & .384 & .033 & 1.1030 & .3933 & .0340 \\
\hline 1.185 & .387 & .042 & 1.1950 & .4274 & .0290 \\
\hline 1.197 & .443 & .033 & 1.2040 & .4300 & $.0<65$ \\
\hline 1.205 & .438 & .029 & 1.2070 & .4380 & .0531 \\
\hline 1.214 & .411 & $.0<4$ & 1.2150 & .4081 & .0274 \\
\hline 1.217 & .407 & .030 & 1.2240 & .4275 & .0263 \\
\hline 1.225 & .427 & $.0<8$ & 1.2270 & .4461 & .0336 \\
\hline 1.233 & .438 & .029 & 1.2350 & .4428 & .0334 \\
\hline $1.23 \leqslant$ & .412 & .035 & $1.24 \cup 0$ & .4015 & .0425 \\
\hline 1.240 & .413 & .001 & 1.2540 & .4133 & .0279 \\
\hline 1.255 & .400 & .050 & 1.2260 & .4173 & .0363 \\
\hline 1.265 & .409 & .050 & 1.2660 & .3924 & .0341 \\
\hline 1.274 & $.4 \%$ & .035 & 1.2700 & $.4 \% 89$ & .0423 \\
\hline 1.283 & $.54 \%$ & .047 & $1.28>0$ & $.51 \cup 8$ & .0547 \\
\hline 1.296 & .520 & .045 & $1.3 \cup>0$ & .4811 & .0523 \\
\hline 1.307 & .490 & .0 .56 & $1.31<0$ & .4640 & .0349 \\
\hline 1.313 & .451 & .039 & 1.5120 & .4056 & .0495 \\
\hline 1.325 & .408 & .043 & 1.5300 & .3912 & .0586 \\
\hline 1.353 & .430 & $.0<9$ & 1.5540 & .4259 & .0319 \\
\hline 1.356 & .424 & .056 & 1.5650 & .4256 & .0522 \\
\hline 1.366 & .420 & .057 & 1.3700 & .4178 & .0367 \\
\hline 1.374 & $.36 \%$ & .038 & 1.5860 & .4403 & .0385 \\
\hline 1.395 & .448 & .034 & 1.4010 & .4465 & .0306 \\
\hline 1.395 & $.4<9$ & .046 & 1.4000 & .3864 & .0336 \\
\hline 1.413 & .400 & .034 & 1.4150 & .3860 & .0410 \\
\hline 1.426 & .399 & .004 & 1.4350 & .4010 & .0348 \\
\hline 1.435 & .382 & .040 & $1.44 \% 0$ & $.38 / 3$ & .0291 \\
\hline 1.453 & .387 & .026 & 1.4550 & .3827 & .0289 \\
\hline 1.456 & .369 & .032 & 1.4040 & .3931 & $.0<97$ \\
\hline 1.466 & .417 & .036 & 1.4730 & .4069 & .0353 \\
\hline 1.475 & .384 & .040 & 1.4660 & .4052 & .0351 \\
\hline 1.490 & .406 & .043 & 1.4900 & .3950 & .0593 \\
\hline
\end{tabular}


TABLE VIII. Cross Sections for the Inelastic Neutron Excitations of the $930-\mathrm{keV}$ State in Vanadium

\begin{tabular}{lll}
\hline $\mathrm{E}_{\mathrm{n}}, \mathrm{MeV}$ & $\sigma_{\text {in }}, \mathrm{b}$ & $\mathrm{d} \sigma_{\mathrm{in}}, \mathrm{b}$ \\
\hline 1.250 & .094 & .057 \\
1.300 & .0850 & .0186 \\
1.350 & .175 & .040 \\
1.360 & .1603 & .0693 \\
1.400 & .145 & .021 \\
1.4100 & .1025 & .0258 \\
1.450 & .125 & .019 \\
1.4650 & .1438 & .0673 \\
1.500 & .998 & .027 \\
\hline
\end{tabular}




\section{ACKNOW LEDGMENTS}

We acknowledge the assistance of a number of members of Argonne National Laboratory staff. We are particularly indebted to Dr.P.A. Moldauer for theoretical assistance and guidance. 


\section{REFERENCES}

1. H. Feshbach, "Doorway States," Proceedings of Conference on Nuclear Structure Study with Neutrons, North-Holland Publishing Company, Amsterdam (1966). See also $\mathrm{H}$. Feshbach et alo, Intermediate Structure and Doorway States in Nuclear Reactions, Ann. Phys. 41, 230 (1967)。

2. P. Hodgson; Optical Model of Elastic Scattering, Oxford University Press, London (1963)。

3. W. Hauser and H. Feshbach, The Inelastic Scattering of Neutrons, Phys. Rev. 87, 366 (1952)。

4. P. A. Moldauer, Average-Compound Nuclear Cross Sections, Rev. Mod. Phys. 36, 1079 (1964).

5. T. Ericson, A Theory of Fluctuations in Nuclear Cross Sections, Ann. Phys. 23, 390 (1963).

6. A. Agodi and G. Pappalardo, Statistical Fluctuations of Compound Nucleus Level Density and Nuclear Cross Sections, Nucl. Phys. 47, 129 (1963).

7. A. Carlson and H. Barschall, Fluctuations in Neutron Total Cross Sections, Phys. Rev. 158, 1142 (1967)。

8. K. Takeuchi, Doorway State Theory of Neutron Nucleus Reactions, Thesis, The University of Michigan, Ann Arbor, Michigan (1968)。

9. R. Lipperheide, A Semi-Phenomenological Nuclear Optical Model Potential, Z. Physik 202, 58 (1967)。

10. Evaluated Nuclear Data FiZe-B, ENDF-B, National Neutron Cross Section Center, Brookhaven National Laboratory (1968).

11. A. B. Smith et al., Multi-Angle Fast Neutron. Time-of-flight System, Nucl。Inst。Methods 50, 277 (1967).

12. J. Whalen, A Digital Computer System for the Executive Control of a Fast Neutron Laboratory, Nucl. Instr. Methods 39, 185 (1966)。

13. A. O. Hanson and J. L. McKibben, A Neutron Detector Having Uniform Sensitivity from $10 \mathrm{keV}$ to $3 \mathrm{MeV}$, Phys. Rev。 72, 673 (1947).

14. A. Langsdorf, Jr., R. O. Lane, and J. E. Monahan, Neutron Scattering Angular Distribution, ANL-5567 (Rev) (Oct 1961). See also, A. Langsdorf, $\mathrm{Jr}_{0}$, et al., Angular Distributing of Scattered Neutrons, Phys. Rev. 107, 1077 (1957).

15. E. Barnard et al., High Resolution Fast Neutron Cross Section of Iron, Nuc1。 Phys. A118, 321 (1968)。

16. J. Gibbons and H. Newson, Fast Neutron Physics, Vo1.'1, edited by

$\mathrm{J}$. Marion and J. Fowler, Interscience Publishers, New York, p. 133 (1960)。

17. J. Stehn et al., Neutron Cross Sections, Brookhaven National Laboratory, BNL-325, Second Edition (1958). See also Second Supplement, Vol.11A, M. Goldberg et al. (1966).

18. J. Cabe et al., Recherche d'Une Structure Intermediaire dans les Sections Efficaces Totales Neutroniques, Nuc1。 Phys. A102, 92 (1967). 
19. G. Rohr and E。 Friedland; A Study of Neutron Resonances of Vanadium and Manganese, Nucl. Phys: A104, 1 (1967).

20. F. Firk et al., Analysis and Interpretation of the Neutron Cross Section of Vanadium below $25 \mathrm{keV}$, Proc. Phys.Soc. 82, 477 (1963).

21. A. G. Guseinov, AnguZar Distribution of Fast Neutrons Scattered by Medium and Heavy Nuclei, translated from At. Energ. (USSR) 18, No. 4, 409 (1965)。

22. Nuclear Data Sheets, National Research Council (1963), detailed references contained therein. See also B。 O'Brien, The Level Structure of $V^{51}, \mathrm{Mn}^{53}$, and $\mathrm{CO}^{55}$ by $\left({ }^{3} \mathrm{He}, \mathrm{d}\right)$ Reactions, Nucl. Phys. A104, 609 (1967); M. Mazari et al., Excited States of $V^{51}$ and $\mathrm{Cr}^{53}$, Phys. Rev. 112, 1691 (1958); and $M_{0}$ Lopez et al。, $A 50 \mathrm{~V}(d, p)$ Experiment, Nuc1. Phys. A94, 673 (1967)。

23. Ho J. Hausman et al, Energy Levels In Magnesium, Vanadium, Chromium, and Manganese, Phys. Rev。88, 1296 (1952).

24. S。 Ofer, and R. Wiener, Decay of Cr ${ }^{51}$, Phys. Rev. 107, 1639 (1957).

25. J. Towle, private communication; see also Paper No. 37, "Neutron Inelastic Scattering from $\mathrm{V}^{51}$ and $\mathrm{Y}^{89}$," Proceedings of Conference on Study of Nuclear Structure with Neutrons, Antwerp (1965), North-Holland Publishing Company, New York.

26. N. Auerbach, Configuration Mixing, Seniority Mixing, and Stripping Experiments in the $f 7 / 2$ Proton Shell, Phys. Letters 34B, 260 (1967).

27. S. C. Mathur et al., private communication.

28. Do Barrows, A Study of the $\left(n, n^{\gamma} \gamma\right)$ Reactions in $V^{51}$ and $M n^{55}$ and the Reaction F12 $^{2}(d, n)$ 'Ne20, Thesis, University of Kentucky (1965).

29. C. A。 Engelbrecht and H. Fiedeldey, Argonne National Laboratory, private communication.

30. L. Wolfenstein, Conservation of Angular Momentum in the Statistical Theory of Nuclear Reactions, Phys. Rev.82, 690 (1951).

31. F. Perey, "The Optical Mode1," Proceedings of Conference on Nuclear Structure Study with Neutrons, North-Holland Publishing Company, Amsterdam, p. 418 (1966).

32. P.A. Moldauer, Optical Model of Low Energy Neutrons Interaction with Spherical Nuciei, Nucl. Phys. 47, 65 (1963).

33. E. Auerbach, Progrom Operation and Input Description, Brookhaven National Laboratory, BNL-6562, ABACUS II (revised version) (Nov 1962); Also P. A. Moldauer, Co.A. Engelbrecht, and G。 J。 Duffy, NEARREX, A Computer Code for Nuclear Reaction Calculations, ANL-6978 (Dec 1964).

34. J. Harvey, as quoted by K. Seth, Nucl. Data 2, No. 3 (1966).

35. P. A. Moldauer, Theory of Average Neutron Reaction Cross Sections in the Resonance Region, Phys. Rev. 123, 968 (1961).

36. L. Galloway and E. Shrader, private communication.

37. R. Becker et al., Elastic Scattering of $3.2 \mathrm{MeV}$ Neutrons from Many Elements, Nucl。Phys. 89, 154 (1966)。 
38. B. Holmqvist. and T. Wiedling, A Systematic Investigation of Fast Neutron Elastic Scattering, Neutron Cross Section \& Technology, Proceedings of Conference; Washington, D.C., March 4-7; 1968, Vol. I, p. 845 .

39. Western et al., Elastic Neutron Scattering at. $14.0 \mathrm{MeV}$, General Dynamics, Fort Worth, AFWL-TR-65-216, Vo1. II。

40. L. Rosen et al., Calculations of Neutron Cross Sections Using a Local Optical Potential with Average Parameters, Los Alamos National Laboratory, LA-3538'(July 1966).

41. T. Ericson and T. Mayer-Kuckuk, Fluctuations in Nuclear Reactions, Ann。Rev. Nucl。Sci。, p. 183 (1966).

42. T. Ericson, On Probability Distribution of Nuclear Cross Sections, Phys. Letters 4, 258 (1963)。

43. P. P. Singh et.al., Frequency of Spurious "Intermediate Resonances" in Randomly Generated Cross Sections, Phys。Letters.23, 255 (1966).

44. G. Pappalardo, Statistical Fluctuations and Intermediate Stmeture in Nuclear Reaction Cross Sections, Phys. Letters 13, 320 (1964).

45. I. Hall, Finite Energy Range and Modulation Effects in the Analysis of Cross Section Fluctuation Data, Phys。Letters 10, 199 (1964).

46. I. Hall, Analysis of Cross Section Fluctuation Data, Oxford University, Nuclear Physics Laboratory Report, 37-64 (1964).

47. H. Vonach and JoHuizenga, Determination of Nuclear Level Densities at an Excitation Energy of $20 \mathrm{MeV}$, Phys. Rev。138, No.6B, 1372 (1965).

48. Go: Calvi et a $\mathrm{Z}_{0}$, Total Cross Sections of $\mathrm{Na}, \mathrm{Mg}$, and $\mathrm{Si}$ for $2.8-5.2 \mathrm{MeV}$ Neutrons, Nucl。 Phys. 48, 408 (1963)。

49. D. W. Glasgow and D. G. Foster, Fast-Neutron Total Cross Sections of. 44 Elements, Hanford Laboratory, HW-SA-2875 (April 1963).

50. N。 Bohr and J.A. Wheeler, The Mechanism of Nuclear Fission, Phys. Rev. 56, 426 (1939)。

51. D. Brink, "Cross-section Fluctuations and Intermediate Structures," Proceedings of Conference on Nuclear Stmicture Study with Neutrons, North-Holland Publishing Company, Amsterdam, p。 278 (1966).

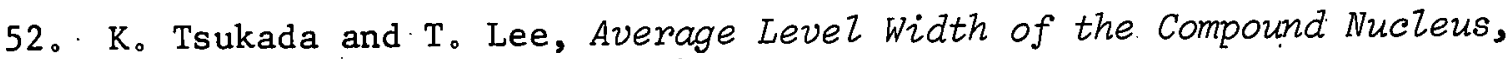
Phys. Letters 11, 141 (1964).

53. $\mathrm{K}_{0} \mathrm{~K}_{0}$ Seth et al., $s_{2} \cdot p$-, and d-Wave Neutron Strength Functions, Phys。Letters 13,70 (1964).

54. A. Gilbert and A. G. W. Cameron, A Composite Nuclear-Level Density Formula with Sheiz Corrections: Can。 J. Phys.43, 1446 (1965).

55. H. A。 Bethe, Nuclear Physics (B) Nuclear Dynomics, Theoretical, Rev. Mod. Phys. 9, 69 (1937).

56. C. E. Porter and R. G. Thomas, Fluctuations of Nuclear Reaction Widths, Phys. Rev. 104, 483 (1956).

57. M. A. Preston, Physics of the Nucleus, Addison-Wesley. Publishing Company, Inc。(1962). 
58. F。 Dyson and M. Mehta; Statistical Theory of the Energy Levels of Complex System. IV, J. Math. Phys. 4, 701 (1963).

59. P.A. Moldauer, Interfering Resonances, ANL-7467' (July 1968).

60. L. S. Rodberg, Intermediate Structure in Nuclear Reactions, University of Kentucky Press, Lexington, Kentucky, p。65 (1967).

61. $\mathrm{R}_{0} \mathrm{H}$ 。 Lemmer, Intermediate Structure in Nuclear Reactions, University of Kentucky Press, Lexington, Kentucky, p。19 (1967).

62. H. Feshbach, Unified Theory of Nuclear Reactions; Ann. Phys. 5, 357 (1958)。

63. H. Feshbach, A Unified. Theory of Nuclear Reactions-II, Ann. Phys. 19, 287 (1962)。

64. R. Lipperheide, On the Nuclear Optical-Model Potential, Nuc1. Phys. 89, 97 (1966).

65. R。 H. Davis, Symposium on Recent Progress in Nuclear Physics with Tandems, Heidelberg, Germany (1966), Session 5-A.

66. P。A. Moldauer, Argonne National Laboratory, private communication.

67. J. Monahan and A. Elwyn, Relation between Fine \& Intermediate Structures in the Scattering of Neutrons by Fe, Phys. Rev. Letters 20 , 1119 (1968).

68. E. M。 Pennington and J. C. Gajniak, Compilation of ENDF-B Data for Magnesium, Titanium, Vanadium, Molybdenum, and Gadolinium, ANL-7387, (March 1968).

69. A. Lejeune and. C. Mahanx, Fine Structure of a Doomway State in $P b^{207}$. Z. Phys。207, 35 (1967).

70. R. H. Lemmer and C. M. Shakin, Resonarlce Reactions and the Nuclear She ZZ Modez, Ann。 Phys. 27, 13 (1964).

71. K。 Takeuchi (to be published)。 